\title{
EZH2-GATA6 axis in
}

\section{Pancreatic ductal adenocarcinoma}

\section{DISSERTATION}

for the award of the degree

"Doctor of Philosophy (Ph.D)"

Division of Mathematics and Natural Sciences

of the Georg-August-Universität Göttingen

within the doctoral program "Molecular Medicine"

of the Georg-August University School of Science (GAUSS)

Submitted by

Shilpa Patil

Born in

Manipal, India

Göttingen, May 2020 



\section{Members of the thesis committee:}

PD. Dr. med. Elisabeth Hessmann (Thesis supervisor \& $1^{\text {st }}$ Reviewer) Department of Gastroenterlogy and Gastrointestinal Oncology University Medical Center, Göttingen

Prof. Dr. Frauke Alves ( $2^{\text {nd }}$ Reviewer)

Translational Molecular Imaging Group

Max Planck Institute of Experimental Medicine, Göttingen

Prof. Dr. rer. nat. Dieter Kube

Department of Hematology and Oncology

University Medical Center, Göttingen

\section{Extended examination Board Members:}

Prof. Dr. med. Matthias Dobbelstein

Institute of Molecular Oncology

University Medical Center, Göttingen

Prof. Dr. med. Heidi Hahn

Institute of Human Genetics

University Medical Center, Göttingen

PD. Dr. rer. nat. Laura Zelarayan-Behrend

Institute of Pharmacology \& Toxicology

University Medical Center, Göttingen

Date of submission of thesis: 07.05 .2020

Date of the oral examination: 22.06 .2020 

DEDICATION

\section{To my parents}

for giving me the space to

dream and fly.

I owe them everything I achieve in my life. 

TABLE OF CONTENTS

ACKNOWLEDGMENTS

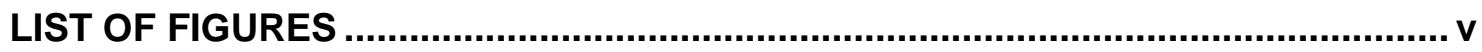

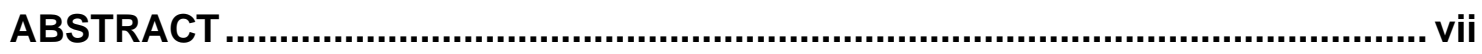

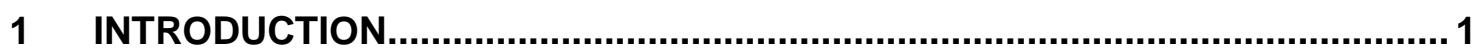

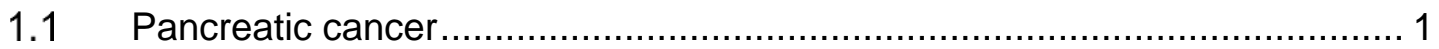

1.1.1 Pancreatic cancer statistics ………………................................. 1

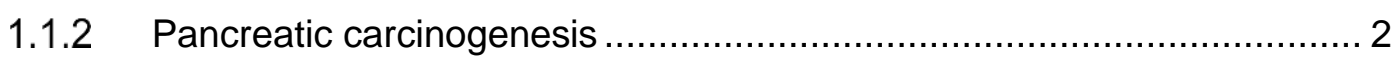

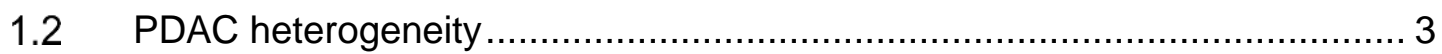

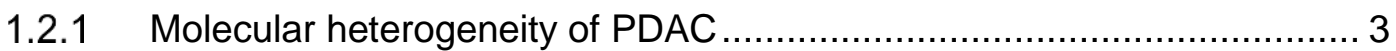

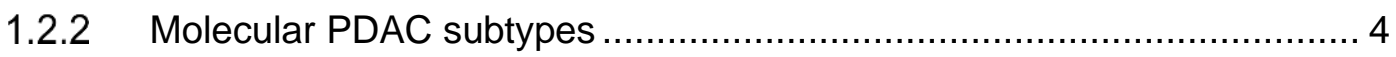

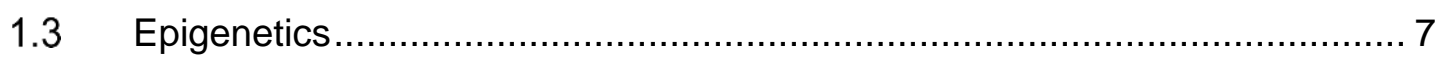

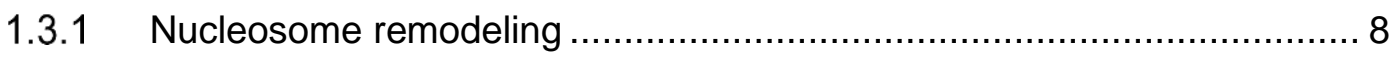

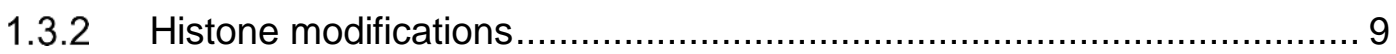

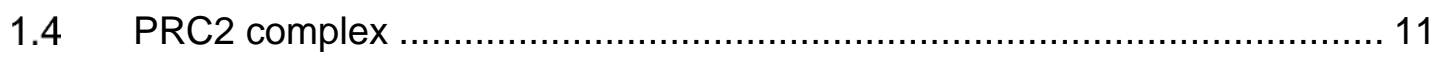

1.4.1 Regulation of EZH2 expression in cancer ........................................ 13

1.4.2 Context-dependent roles of EZH2 ................................................... 14

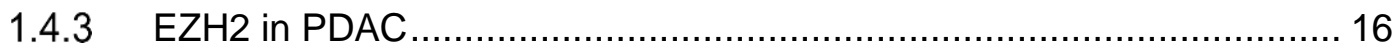

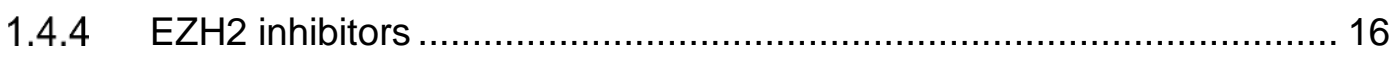

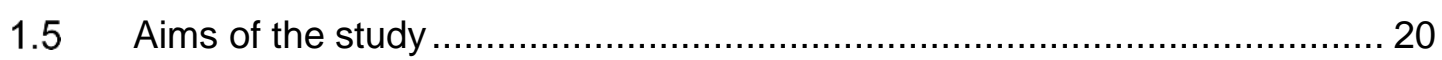

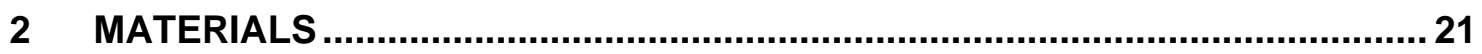

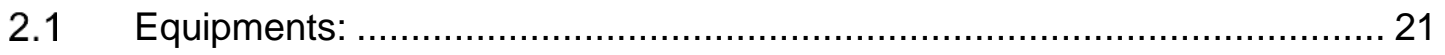

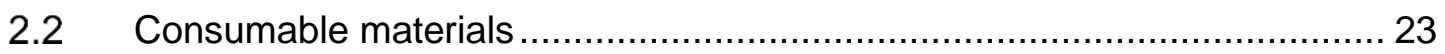

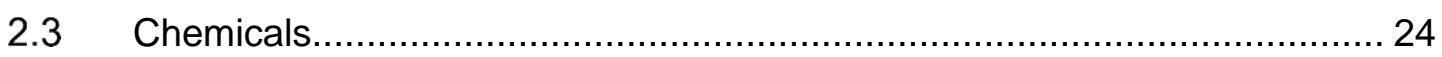

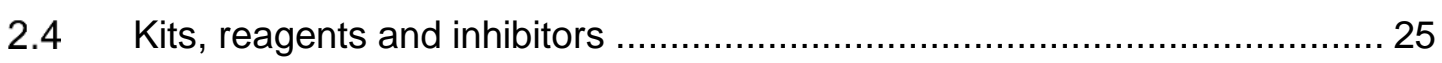

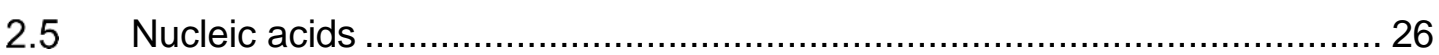

2.5.1 gRNA sequences and validation primers for CRISPR/Cas9-mediated

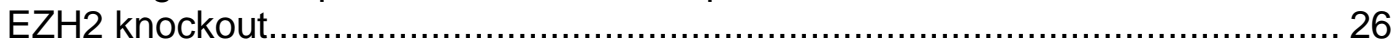

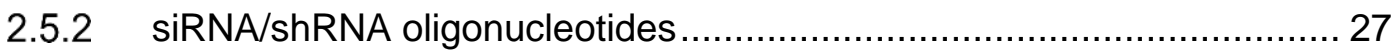

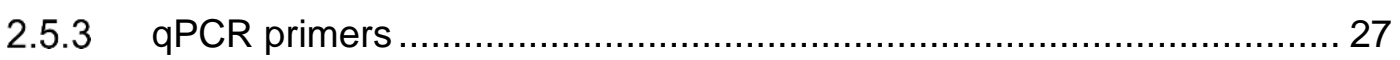

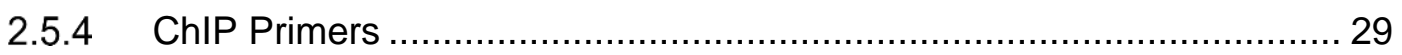

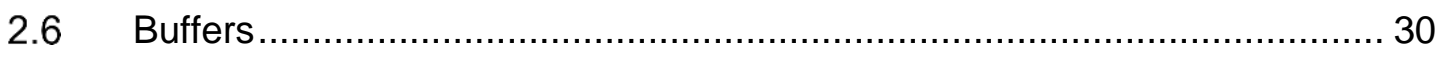

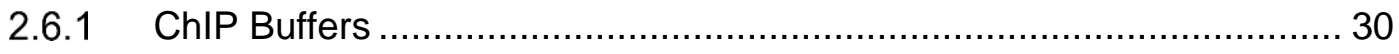

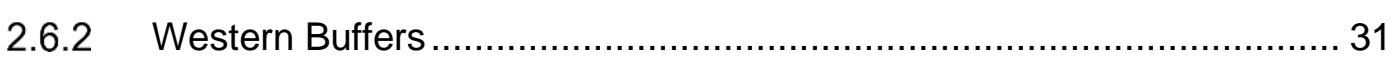

2.7 Antibodies, Enzymes and standards ..................................................... 34

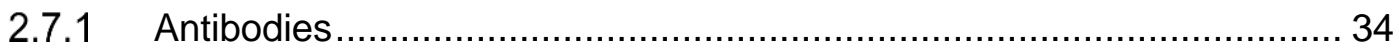

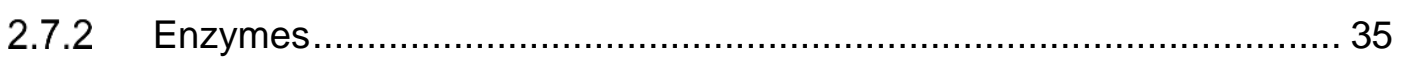




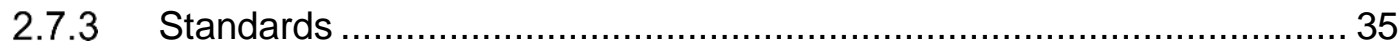

2.8 Cell culture reagents, growth media and antibiotics ................................ 35

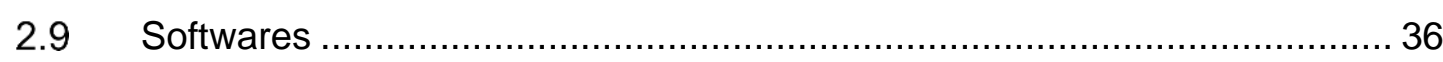

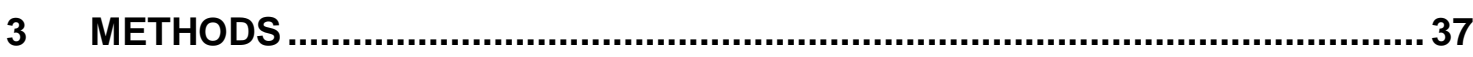

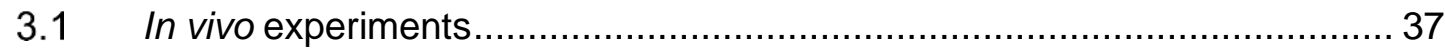

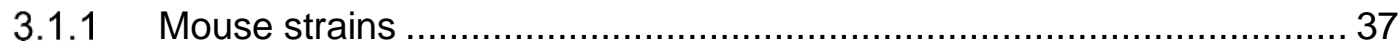

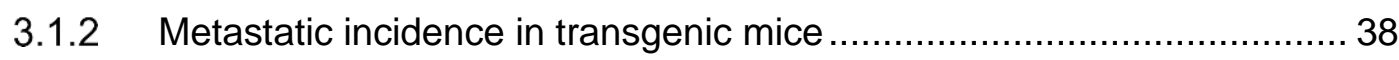

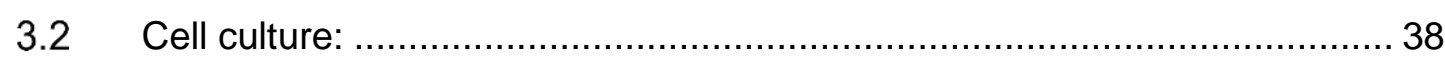

3.2.1 Cells, culture conditions and Tazemetostat (EPZ6438) treatment ......... 38

3.2.2 Generation of EZH2 shRNA and CRISPR/Cas9 clones and transient

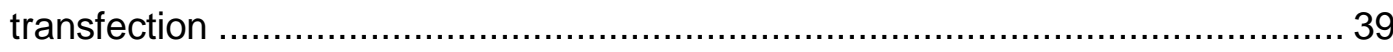

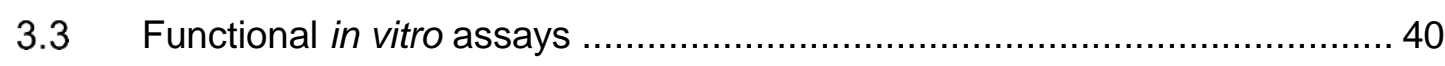

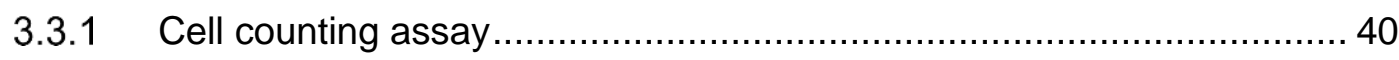

3.3.2 BrdU (Bromodeoxyuridine) assay ................................................. 40

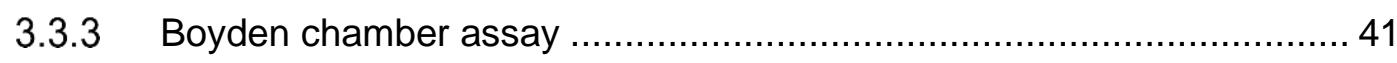

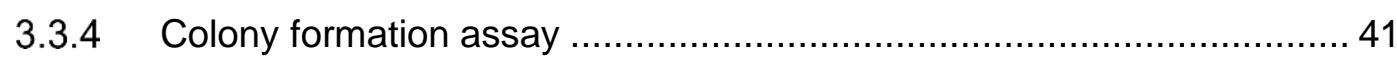

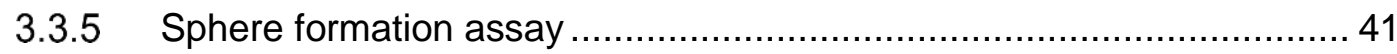

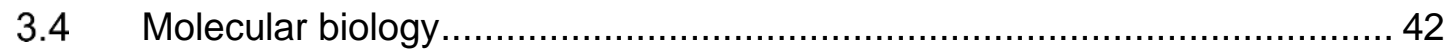

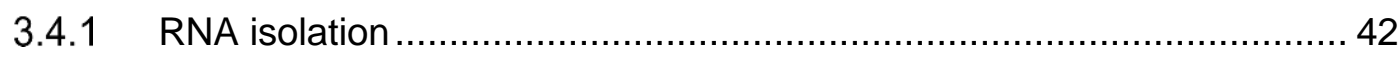

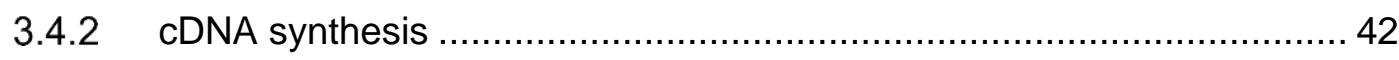

3.4.3 Quantitative polymerase chain reaction (qPCR) ............................ 42

3.4.4 Chromatin Immunoprecipitation (ChIP) ...................................... 43

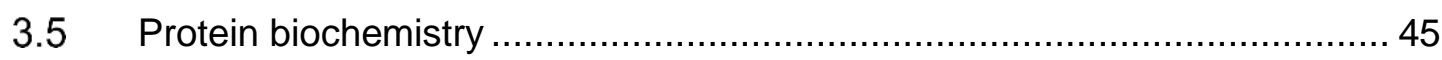

3.5.1 Sample preparation and Bradford Assay ....................................... 45

3.5.2 SDS PAGE and Western blot analysis ............................................ 45

3.6 Next generation sequencing: ......................................................... 46

3.6.1 ChIP seq library preparation and analysis ...................................... 46

3.6.2 RNA seq library preparation and analysis ................................... 47

3.7 H\&E staining and immunohistochemistry ............................................. 48

3.8 Tissue Microarray Analysis (TMA) in human PDAC samples ..................... 49

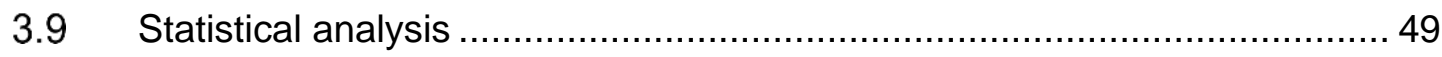

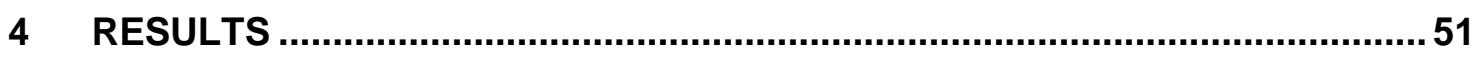

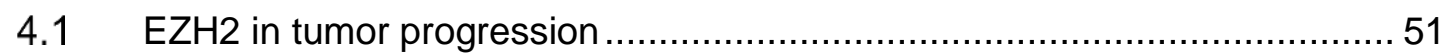

4.1.1 $E Z H 2$ is overexpressed in dedifferentiated human PDAC ................... 51

4.1.2 EZH2 drives PDAC progression in transgenic mice ............................ 52

4.1.3 EZH2 drives liver metastasis in transgenic mice ............................. 57

4.2 Functional implications of EZH2 in PDAC cells ....................................... 58

4.2.1 EZH2 depletion reduces proliferation in NKC cells ............................ 60

4.2.2 EZH2 depletion reduces stemness properties in NKC cells..................61 61 
4.2.3 EZH2 depletion reduces the invasive potential of NKC cells 62

4.3 Mechanistic role of EZH2 in PDAC

4.3.1 EZH2 regulates genes mediating differentiation and tumor progression in PDAC 64

4.3.2 EZH2 depletion leads to enrichment of favorable prognosis and differentiation gene sets

4.3.3 Classical subtype PDAC genes are positively enriched upon EZH2 depletion .....

4.3.4 EZH2 binds largely on TSS/promoter sites of its target genes

4.3.5 EZH2-deficiency fosters a shift in histone modifications at EZH2 target genes 71

4.3.6 EZH2 targets differentiation-associated genes................................ 73

4.3.7 EZH2 target gene expression validation ...................................... 75

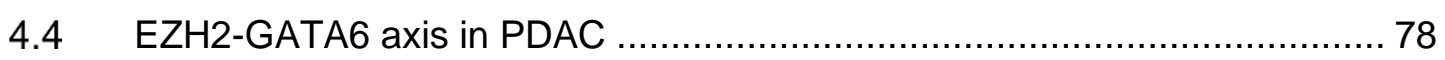

4.4.1 EZH2 targets epithelial transcription factor Gata6 ............................... 78

4.4.2 EZH2 depletion significantly increases Gata6 expression in murine

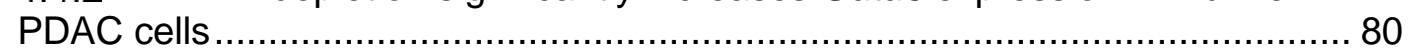

4.4.3 EZH2 represses GATA6 expression in human PDAC .......................... 82

4.4.4 The EZH2-GATA6 axis exists in human PDAC specimens .................. 84

4.4.5 GATA6 targets are enriched upon EZH2 depletion ............................. 85

4.4.6 Gata6 knockdown can rescue the effects of EZH2 depletion on invasion86

5 DISCUSSION.

5.1 Polycomb group proteins in the regulation of differentiation processes....... 89

$5.2 \quad \mathrm{EZH} 2$ in pancreas development and cancer progression ......................... 89

5.3 Role of epigenetic modulations in mediating cancer cell plasticity .............. 92

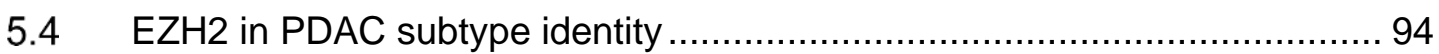

5.5 GATA6 as a marker of the classical PDAC subtype .................................. 96

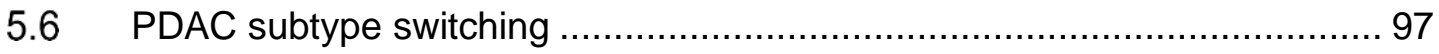

5.6.1 Strategies to induce GATA6 expression for subtype switching..............98

5.7 Pharmacological inhibition of EZH2 in PDAC ..................................... 100

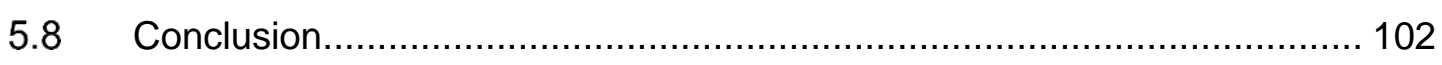

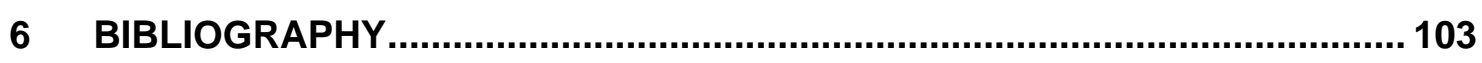

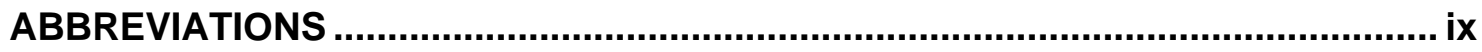

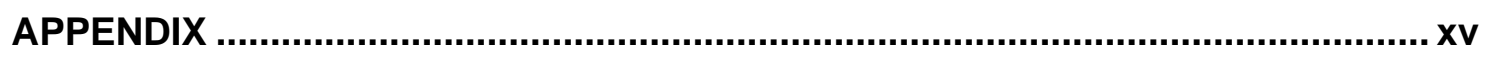

CURRICULUM VITAE ...................................................................................... xvii 



\section{ACKNOWLEDGMENTS}

\section{ACKNOWLEDGMENTS}

"No one can whistle a symphony. It takes an orchestra to play it" - H.E.Luccock

Ph.D. is like topography - There are summits of happiness and success, flat stretches of boring routine, valleys of frustration and failures. Now when I look back, it feels completely worth experiencing the walk. The last four years have been amazing years of immense learning and personal growth. Though I stand alone on the cover of this thesis, the credit goes to many people for helping me persevere and complete my degree with satisfaction. I would like to acknowledge all the people who have been a major part of my journey.

I have been amazingly fortunate to have PD. Dr. med. Elisabeth Hessmann (Lissy) as my supervisor, who has continuously provided me with valuable guidance, encouragement and support throughout these years. She has given me the freedom to pursue my research while also silently ensuring that I stay on track. Apart from science, I have always tried to imbibe from her values on patience, productivity, hard work, balancing life, being humble and extremely grounded. She will always be someone that I would look up to in my life for her exceptional scientific knowledge as well as extraordinary human qualities.

On a special note, I would like to thank Prof. Dr. med. Volker Ellenrieder for giving me the opportunity to work in this young, collaborative and enthusiastic department that he has built and supported for years. I acknowledge the financial support for my research study by Deutsche Krebshilfe and Wilhelm Sander Stiftung funding sources.

I express my deep and sincere gratitude to Prof. Steven Johnsen (Steve) for showing genuine interest in my project, constantly providing credible ideas and being very approachable. I thank Prof. Frauke Alves for all the valuable insights on my project throughout these four years. She never missed to ask me about my problems and wellbeing in Germany especially in my initial days and I greatly appreciate her concern. I also thank Prof. Dieter Kube for joining as my TAC member in between my Ph.D. journey and providing helpful suggestions on my project.

My sincere gratitude goes to Prof. Matthias Dobbelstein for giving me the opportunity to collaborate in his project and for being an extremely inspiring person. I thank him as well as Prof. Heidi Hahn and PD. Dr. rer. nat. Laura Zelarayan for agreeing to be my external examination board members.

I appreciate the support received from our collaborators- Prof. Philipp Ströbel and Dr. Stefan Kueffer from the Institute of Pathology. I thank Dr. Vijayalakshmi Kari, Dr. 


\section{ACKNOWLEDGMENTS}

Zeynab Najafova and specially Xin Wang who was always available to help and teach me bioinformatic analysis as well as for being a very good friend.

I am deeply grateful to Dr. Shiv K. Singh for being immensely helpful at all times and for all the refreshing conversations I have had with him during these four years. I sincerely thank PD. Dr. Dr. med. Albrecht Neesse for introducing me to the ultrasound techniques and giving me an opportunity to be a part of his projects. My special thanks to Dr. med. Robert Goetze (Robby) for patiently making me better at my ultrasound technical skills and for being a very caring, generous and super-friendly person.

I am indebted to Christin Kellner, Jessica Spitalieri and Kristina Reutlinger for the excellent technical support, and Sercan Mercan and Tobias Kaiser for their generous help with the mice. I am thankful to Ulrike Wegner for always being pleasant and Jutta Blumberg for all her warm hugs which were my instant stress-burster. My very special gratitude goes to Waltraut Kopp for always going an extra mile to help me with mouse studies, experiments and for being extremely kind, affectionate and caring.

I literally have the 'best' Ph.D. mates - Zhe Zhang and Lennart Versemann. I thank them for helping me around in lab, listening to all my experiments-not-working issues, proofreading my thesis, giving constructive feedback and giving me the privilege of friendship to be myself. I thank all my current/former fellow colleagues - Mengyu, Umair, Lucas, Geske, Fredi, Nina, Marie, Melanie, Iswarya and all others who have in their own ways assisted me on this path and have ensured that good times kept flowing. I thank Jan Arne and Joshua Blume for giving me an opportunity to develop my teaching skills.

Cheers to the best friends I made in this lab - Alice, Mengyu and Cezerela. I genuinely value them for their amazing company, never-ending conversations, crazy movie nights, stupid advice, loads of laughter and all the special 'little moments' which will remain the best part of my lab memories.

My days in Goettingen would not have been the same without the company of a few people: I am extremely grateful to Iswarya (Ishu) whom I completely relied on when I initially came to Germany. I thank her for all those times she cooked for me and took care of my health. My heartfelt gratitude goes to Anusha and Sandeep, who are my family away from home. I thank them for always counting me in their life, making me feel a little less homesick and for all the advice they have given me. I thank my buddy, Uday, whom I can always count on and fight with. My special gratitude goes to Maithily (Maithy). I was very fortunate to have met her when we both newly arrived in Germany and we have figured out everything together since then. Our every weekend travels, 


\section{ACKNOWLEDGMENTS}

Europe-trips, dance practices and laughing at our own jokes have kept me sane in Goettingen. I am very grateful for her continuous moral and emotional support and hope this to continue in the future.

Back home, I extend my gratitude to my amazing bunch of school friends who have been there in all aspects of my ups and downs for the last 18 years. I treasure the lovely friendships I have made along the way - Sunaina, Stephanie, Sidharth, Sunita, Revathi and my forever enthusiastic friend Sushma. I am deeply thankful to these people for making my bad times bearable and good times even better. I would like to specially thank Shikha who has always given me the extra push I needed and has so far proofread all my thesis, applications, emails, CV and so on since my masters. I can blindly count on her to continue being my support system in the future.

I am extremely grateful to my Master thesis supervisor Dr. Sweta Srivastava - a strong reason for me to step deeper into science and research. She has truly been one of my 'woman of impact' and I thank her for the space and liberty she has given me to approach her anytime for any kind of advice.

I whole-heartedly thank my best friend, Himanshu, for his every day phone calls, an overwhelming amount of laughter, honest opinion on all my complaints, extraordinary moral support and for his unique way of putting me back on track. All the conversations that I have had with him has been a constant reminder for me to watch my thoughts which has helped me develop a broader perspective in life.

My biggest source of strength and the strongest backing that I could ever wish for is my family. My heartfelt gratitude to my grandmother (doddamma) for her unwavering love and blessings. I am immensely grateful to my parents for their unconditional love, unfiltered trust, endless support, quite patience and silent prayers. My deeply treasured gratitude goes to my most favorite person, my human diary and literally my second mother - my amazing sister, Kripa - whose footsteps I have copied throughout my childhood. I specially thank my brother-in-law, Shravan, for teaching me to give myself the space to figure out what I wanted. He was just a phone call away to help me handle my silly computer-related issues to life- and career-related confusions. I thank them both for taking all my tantrums, handling my stubbornness and for being my eternal cheerleaders. I am blessed to have my family that has never let me feel short of love and care.

Lastly, I want to express my deepest gratitude to my life mentor Dr. A.P.J. Abdul Kalam. Some people leave a huge impact on our life without ever meeting in-person. With his books and speeches, I never felt short of inspiration and motivation. 



\section{LIST OF FIGURES}

\section{LIST OF FIGURES}

Figure 1: Schematic depicting heterogeneity in pancreatic cancer................... 4

Figure 2: Schematics depicting the chromatin regulators. ……..................... 9

Figure 3: Schematic illustrating the function mediated by the PRC2 complex . 12

Figure 4: EZH2 SET-domain inhibitors and their mechanism of action............ 18

Figure 5: EZH2 expression correlates with high grade PDAC. ..................... 52

Figure 6: Complete loss of EZH2 hampers pancreatic development in mice... 53

Figure 7: EZH2 drives PDAC development and dedifferentiation. ................... 55

Figure 8: EZH2 correlates with increased PDAC incidence in transgenic mice 56

Figure 9: EZH2 deficiency reduces metastatic incidence.............................. 57

Figure 10: Generation of shRNA-mediated stable knockdown of EZH2 in NKC

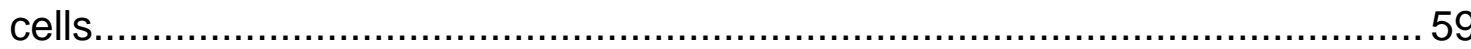

Figure 11: EZH2 depletion reduces cell proliferation in NKC cells.................60

Figure 12: EZH2 deficiency reduces anchorage independent growth and

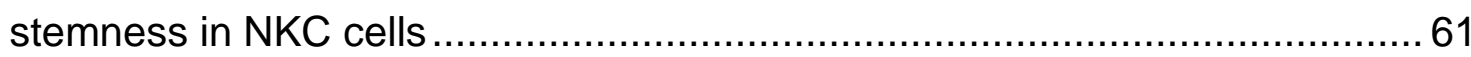

Figure 13: EZH2 knockdown reduces invasive capacity of NKC cells. ............ 62

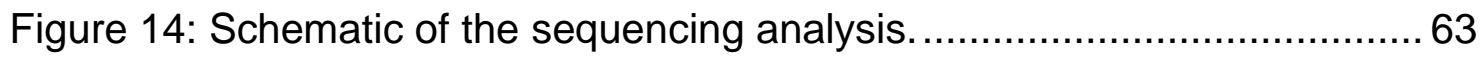

Figure 15: EZH2 deficiency leads to enrichment of pathways related to PDAC

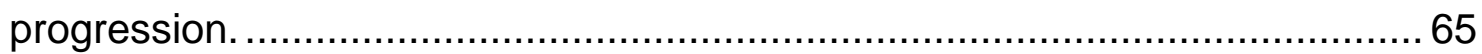

Figure 16: EZH2 depletion mediates better prognosis and cell differentiation programs 66

Figure 17: EZH2 knockdown leads to positive enrichment of classical PDACand negative enrichment of basal-like PDAC subtype gene signatures. 68 Figure 18: EZH2 knockdown leads to enrichment of lipogenic subtype gene signatures.

Figure 19: EZH2 largely occupies the promoter region of its target genes. ..... 71

Figure 20: PCA plots of histone marks from ChIP sequencing analysis ......... 72 Figure 21: Loss of EZH2 binding leads to decrease in $\mathrm{H} 3 \mathrm{~K} 27 \mathrm{me} 3$ and increase in H3K4me3 enrichment. ...................................................................... 73

Figure 22: Direct target genes of EZH2 mediate developmental and cell

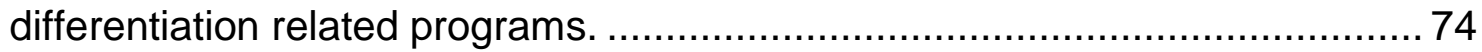

Figure 23: IGV profile of selected 11 EZH2 direct target genes..................... 75 


\section{LIST OF FIGURES}

Figure 24: Expression of direct EZH2 target genes increases upon EZH2 depletion.

Figure 25: EZH2 binds to TSS of its target genes and mediated promoter specific histone modifications. 78

Figure 26: EZH2 binds to TSS of Gata6 and mediates TSS specific histone modifications. 79

Figure 27: EZH2 deficiency leads to increased GATA6 expression in murine PDAC cells

Figure 28: EZH2 regulates GATA6 expression in human PDAC cell line PANC1.

Figure 29: EZH2 regulates GAT6A expression in cells derived from PDX models. 83

Figure 30: EZH2 dependent GATA6 regulation is evident in the subpopulation of PDAC patients. 84

Figure 31: Direct downstream targets of GATA6 are enriched upon EZH2 depletion

Figure 32: GATA6 knockdown partially rescues the invasion effects mediated by EZH2 deficiency.

Figure 33: Schematic depicting two main PDAC subtypes and its features. ....94

Figure 34: Proposed model depicting subtype switching in PDAC mediated by inhibiting EZH2.

Figure 35: Generation of CRISPR/Cas9 mediated stable knockout of EZH2 in NKC cells. $x v i$ 


\section{ABSTRACT}

Pancreatic ductal adenocarcinoma (PDAC) is a highly heterogeneous disease which is significantly driven by epigenetic alterations, e.g. installed by overexpression of Enhancer of Zeste homologue 2 (EZH2). EZH2 constitutes the catalytic member of the PRC2 complex and plays a pivotal role in gene repression by mediating histone methylation (H3K27me3). High EZH2 expression levels are predominantly found in undifferentiated PDAC tumors and are associated with poor prognosis. However, the underlying mechanisms that bridge EZH2 activity to PDAC dedifferentiation remain elusive. Here we aim to dissect the mechanistic and functional role of EZH2 in PDAC progression and dedifferentiation.

The functional implications of EZH2 are investigated in vitro in primary PDAC cells upon genetic (CRISPR/Cas9 and shRNA-based) or pharmacological inhibition (EPZ6438) of EZH2 and in vivo utilizing transgenic mice of EZH2 deficiency and Patient-Derived-Xenograft (PDX) models. EZH2-dependent gene signatures are identified based on ChIP-and RNA-seq analyses and are further validated by independent gene expression studies.

EZH2 activity correlates with increased tumor incidence and metastatic propensity in murine PDAC and drives dedifferentiation in human PDAC. Blockade of the histone methyltransferase reduces proliferation, invasion and stemness features in PDAC cells. Accordingly, genome wide binding- and expression analyses reveal EZH2 as a repressor of differentiation-associated gene signatures and indicate that blockage of EZH2 activity induces a gene signature shift towards classical and less aggressive molecular PDAC subtypes. The endodermal transcription factor encoding GATA6 gene is identified as one of the most significantly regulated direct EZH2 targets. Consequently, abrogation of GATA6 upregulation in the context of EZH2deficiency partially counteracts the acquisition of classical gene signatures and reinstalls their invasive capacities of PDAC cells, suggesting that the tumorigenic activity of $\mathrm{EZH} 2$ is critically determined by GATA6 repression. 


\section{ABSTRACT}

Together, our findings link the EZH2-GATA6 axis to PDAC subtype identity and suggest EZH2 inhibition as a promising therapeutic strategy to induce subtypeswitching in favor of a less aggressive PDAC phenotype. 


\section{INTRODUCTION}

\section{INTRODUCTION}

\subsection{Pancreatic cancer}

The pancreas is a glandular organ that lies behind the lower part of the stomach in the abdomen. It mainly consists of two functional units - endocrine and exocrine. The endocrine cells help regulate the blood glucose levels, and the exocrine unit, which is made of acinar and ductal cells, produces and releases digestive enzymes (Zhou and Melton 2018). The tumor can form in cells from any of these compartments but $90 \%$ of the pancreatic cancers are pancreatic ductal adenocarcinoma (PDAC), which arise from the acinar cells of the pancreas (Iguchi et al. 2016).

\subsubsection{Pancreatic cancer statistics}

Pancreatic cancer is currently the third leading cause of cancer-related deaths in the United States (Tavakkoli et al. 2020) and fourth in Europe (Ferlay et al. 2018). With the records of increasing incidence, consistently low survival rates and no significant improvements in mortality trends, it is projected to be the second leading cause of cancer-related deaths by 2030 (Tavakkoli et al. 2020). According to GLOBOCAN 2018 estimates, pancreatic cancer is the $11^{\text {th }}$ most common cancer in the world and was reported to cause 432,242 deaths $(4.5 \%$ of all deaths caused by cancer) in 2018 (Bray et al. 2018). With the highest mortality rate of $91 \%$, to date, it remains one of the deadliest gastrointestinal malignancies worldwide. Even though the overall incidence of pancreatic cancer is relatively less, the incidence almost equals the mortality rate (GordonDseagu et al. 2018; Rahib et al. 2014). Despite all the scientific efforts in the last few decades, the five-year survival rate of PDAC remains at $9 \%$, which is the lowest of all cancer entities (Rawla, Sunkara, and Gaduputi 2019). These alarming statistics are reasoned by late diagnosis due to lack of specific early detection methods and no predictive symptoms or nonspecific symptoms. Furthermore, tumors are unresectable when detected at an advanced stage. PDAC displays a high recurrence rate after surgery and an intrinsic resistance against diverse therapeutic regimens, mainly because of untailored therapeutic strategies, extensive tumor heterogeneity and dynamic cellular plasticity (Orth et al. 2019). Approximately $70 \%$ of the patients do not respond to therapies 
and additionally upon failure of chemotherapy, they develop accelerated tumor growth, resistance and metastasis (Juiz, lovanna, and Dusetti 2019). Consequently, advanced research is urgently needed for the better understanding of disease etiology and the development of novel therapeutic strategies.

\subsubsection{Pancreatic carcinogenesis}

The origin of PDAC was deciphered in the $21^{\text {st }}$ century with the development of genetically engineered mouse models (GEMMs) of PDAC. For a long time, PDAC was thought to originate from the ductal cells of the pancreas (J. M. Bailey, DelGiorno, and Crawford 2014; Busnardo et al. 1983). Later, it was experimentally confirmed that PDAC originates from the acinar cells, which are known to possess a high degree of cellular plasticity (J. M. Bailey et al. 2015; Kopp et al. 2012; Ray et al. 2011). In 1988, Smit et al. set a link between a mutation in codon 12 in KRAS and PDAC (Smit et al. 1988). Subsequently, many GEMMs verified the association and confirmed that point mutations in the KRAS gene is the earliest event for the onset of PDAC (Hingorani et al. 2003; Olive and Tuveson 2006). The KRAS gene codes for the KRAS protein, a Guanosine triphisphatase (GTPase), which acts as a switch to regulate the RAS/MAPK signaling pathway. The mutation results in a constitutively active KRAS protein that continuously drives proliferation independent of external stimuli (Ellis and Clark 2000). The KRAS oncogene is found to be mutated in $90 \%$ of PDACs (Hansel, Kern, and Hruban 2003; Hruban et al. 1993), and this gatekeeper KRAS mutation is vital for the initiation of PDAC (Kandha et al. 2012). Studies in mouse models show that upon acinar damage, mutant KRAS blocks acinar regeneration, thus resulting in progression of Acinar-to-ductal metaplasia (ADM) to Pancreatic Intraepithelial Neoplasia (PanIN) formation (Morris et al. 2010). PanINs represent PDAC precursor lesions, which progress through three well-defined stages (PanIN1A/1B, PanIN2, PanIN3) and have the potential to cause PDAC. These lesions differ in their level of cytological abnormalities (Guerra et al. 2007) and are associated with specific step-wise genetic changes. Oncogenic KRAS is present in $90 \%$ of PanIN1A/1B, CDKN2A is found to be inactivated in $90 \%$ of PanIN2 (Caldas et al. 1994; Russo et al. 1998), inactivation of TP53 is found in 50-70 \% (Barton et al. 1991; 


\section{INTRODUCTION}

Berrozpe et al. 1994; Rozenblum et al. 1997; Solcia, Bonato, and Ranzani 1994) and loss of SMAD4 is seen in 55 \% (Hahn et al. 1996) of PanIN3 (Guerra et al. 2007; lacobuzio-Donahue 2012; Thilo Welsch, Jorg Kleeff, and Helmut Friess 2007).

Although oncogenic activation of KRAS is a very early initiating event for PDAC origin, certain environmental stimuli like stress or acinar cell injury accelerate PDAC initiation and further progression (Dumartin et al. 2017). Accordingly, chronic pancreatitis displays a crucial risk factor for PDAC development (Yadav and Lowenfels 2013). These events ultimately enhance the secretion of inflammatory factors which in combination with mutant KRAS boost progression of PDAC (Baer et al. 2014; Ye et al. 2019; Young et al. 2019).

\subsection{PDAC heterogeneity}

\subsubsection{Molecular heterogeneity of PDAC}

One of the major reasons for the aggressive tumor biology and the therapeutic resistance of PDAC is its high molecular heterogeneity. Next-generation sequencing approaches conducted in the last few years have significantly extended our understanding of the molecular complexity of PDAC. The first whole-exome sequencing experiment in pancreatic cancer specimens conducted in 2008 identified alterations in a number of oncogenes and tumor suppressor genes (Jones et al. 2008). Further, numerous follow-up studies have revealed huge amount of information regarding the wide spectrum of mutational and copy number variations in PDAC (Adamo et al. 2017; P. Bailey et al. 2016). These reports have not only reaffirmed signature mutations in KRAS, CDKN2A, SMAD4 and TP53 genes but revealed additional genetic alterations that occur throughout the process of tumor progression, although at lower prevalence (P. Bailey et al. 2016; Biankin et al. 2012; Du et al. 2017). However, recent work indicates that phenotypic features of PDAC are not only driven by genetic alterations but are significantly determined by epigenetic changes (G. Lomberk et al. 2018; Nicolle et al. 2017). Hence, epigenetic dysregulation critically contributes to the molecular diversity of PDAC. 


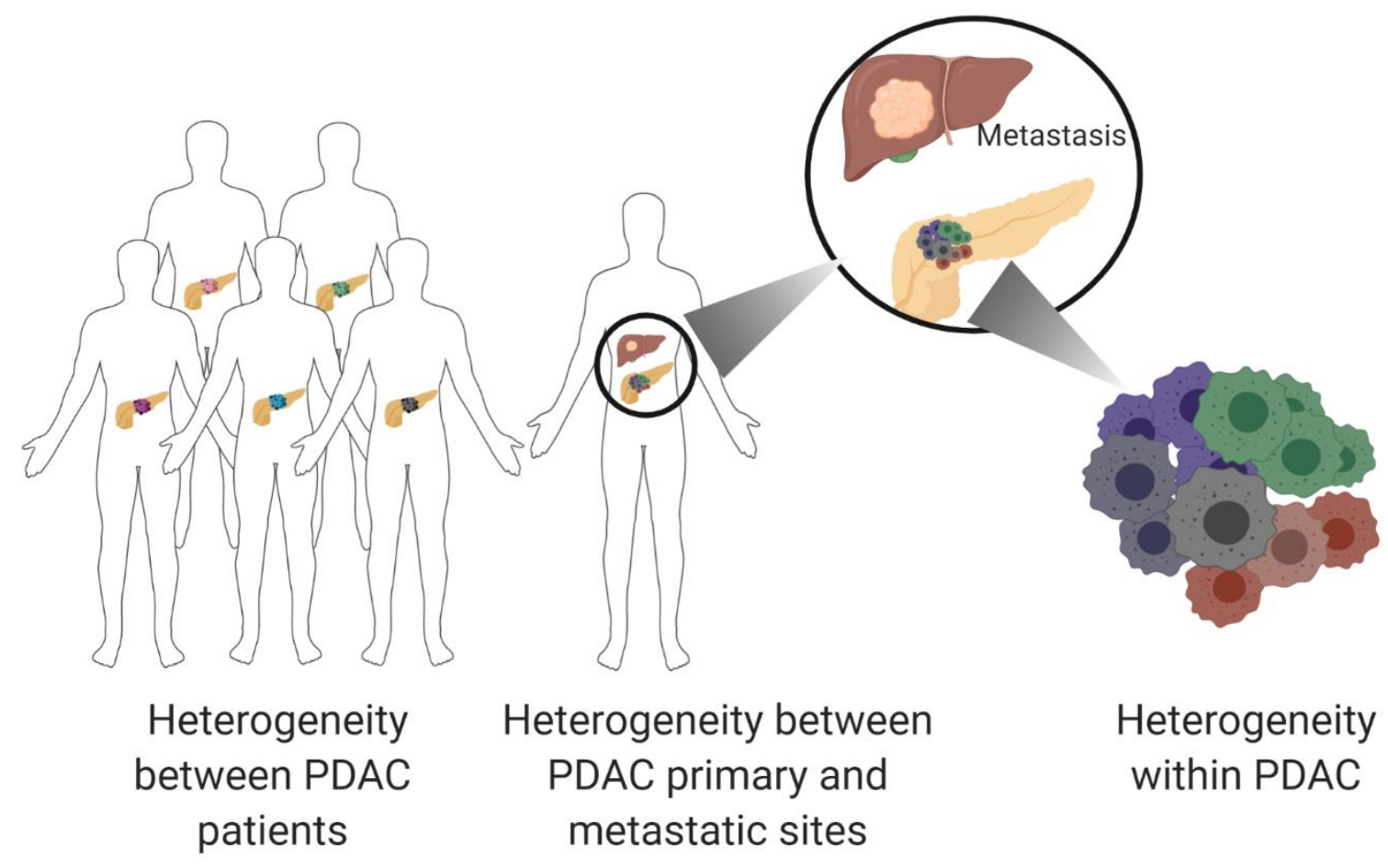

Figure 1: Schematic depicting heterogeneity in pancreatic cancer.

Genetic and molecular heterogeneity exists between different patients (intertumoral heterogeneity), primary and metastatic sites within the same patient as well as within different parts of the same tumor (intratumoral heterogeneity).

\subsubsection{Molecular PDAC subtypes}

The high degree of molecular heterogeneity in PDAC is reflected by the identification of various molecular PDAC subtypes with prognostic and therapy predictive relevance (Aung et al. 2019; P. Bailey et al. 2016; Collisson et al. 2011; Moffitt et al. 2015). The first study aiming at molecular PDAC subtyping was performed by Collisson et al. in 2011 and is based on microarray-based transcriptome analysis in microdissected primary resected PDAC samples as well as in mouse and human cell lines. The authors unraveled three subtypes: classical (increased expression of epithelial genes), QM (quasi-mesenchymal, increased expression of mesenchymal genes) and exocrine (increased expression of digestive enzymes genes). They also show the association of these subtypes with clinical outcome and differences in response to therapy. They developed PDAssigner gene sets comprising 62 genes that define their subtypes. (Collisson et al. 2011). Following this up, in 2014, Kim et al. conducted microarray analysis in 96 resected non-micro-dissected samples 


\section{INTRODUCTION}

and shared similar findings. They found three molecular subtypes: subtype1 (similar to classical subtype with enriched immune pathways), subtype2 (resembles QM PDA) and subtype3 (similar to exocrine-like) which had a significant correlation with tumor size, metastasis and survival (S. Kim et al. 2014).

In 2015, Moffit et al. performed virtual microdissection to separate stroma from epithelial tumors and looked for gene expression in both tumoral and stromal compartment. They conducted microarray analysis in both primary and metastatic samples and further validated their results by RNA-sequencing (RNA-seq), which is a more sensitive method and can capture a broader range (Rodríguez-García, Sola-Landa, and Barreiro 2017). They presented two subtypes for the tumor compartment: classical and basal-like which overlapped with the Collisson classical and QM subtypes, respectively. The authors also distinguished two stromal subtypes: Normal and activated stroma which independently serve as prognostic markers (Moffitt et al. 2015). Soon after this, in 2016, Janky et al. published their microarray data in resected samples, where they found three clusters largely reflecting the findings from Collisson et al (Janky et al. 2016). In 2016, Bailey et al. in collaboration with International Cancer Genome Consortium (ICGC), performed integrated genomic analysis and gene expression analysis using deep-exome sequencing and RNA-seq approaches. They explored 32 significantly mutated genes that aggregated into 10 molecular pathways. Clustering from the gene expression analysis revealed four clinically relevant subtypes: Progenitor, Aberrantly differentiated endocrine exocrine (ADEX), Squamous and Immunogenic. The progenitor and squamous subtypes shared common features with Collisson's classical and QM subtypes, respectively (P. Bailey et al. 2016). However, work from The Cancer Genome Atlas (TCGA) categorized ADEX and Immunogenic subtypes as less pure. Using the transcriptomic data from Bailey et al., Müller classified five different clusters: cluster1 overlapped with squamous subtype, cluster2 had more epithelial cell signatures, cluster3 had signatures related to EMT and MAPK pathways which are characteristics of undifferentiated tumors, cluster4 was similar to the immunogenic subtype and cluster5 resembled ADEX subtype gene signatures (Mueller et al. 2018). 
More recently, Puleo et al. conducted a large investigation of the transcriptional landscape by performing microarray analysis in 309 paraffin-embedded samples. The study integrates data from epithelial and stromal compartments. The unsupervised classification of the whole tumor entity revealed five subtypes: Pure classical, immune classical, desmoplastic, stroma activated and pure-basal-like. The high tumor cellularity classification revealed two welldefined subtypes: classical and basal-like. This study captured the molecular diversification of PDAC compartments and validated the existence of classical and basal subtypes as described in all previous PDAC stratification studies (Puleo et al. 2018). In the follow-up study, Maurer et al. (2019) carried out RNAsequencing in 60 resected samples and revealed two epithelial (classical and basal-like) and two stromal (ECM rich and immune rich) subtypes (Maurer et al. 2019).

In summary, two subtypes exist in the epithelial PDAC compartment, which have been univocally identified by all transcription-based subtyping studies conducted in PDAC so far: classical and basal-like. The classical molecular PDAC subtype is characterized by the expression of epithelial differentiationrelated gene signatures, is less aggressive and displays a relatively good prognosis. In contrast, basal-like PDAC is highly aggressive and is associated with chemoresistance and low survival (P. Bailey et al. 2016; Collisson et al. 2011; G. Lomberk et al. 2018; Moffitt et al. 2015; Puleo et al. 2018). Hence, the aforementioned subtyping studies have not only allowed for a better understanding of the remarkable molecular heterogeneity of PDAC, but have revealed molecular explanations for the poor efficacy of "one-size-fits-all" therapeutic approaches in PDAC treatment. Moreover, these studies clearly emphasize the necessity of considering the diverse molecular PDAC characteristics for pursuing tailored therapeutic strategies to combat pancreatic cancer. Finally, the aforementioned studies indicate that molecular PDAC subtypes are primarily defined at the level of gene transcription. Hence, a thorough understanding of the mechanistic regulation of PDAC subtypes requires the exploration of epigenetic alterations underlying the classification of PDAC tumors. 


\section{INTRODUCTION}

\subsection{Epigenetics}

Epigenetics literally translates to 'outside genetics'. The term epigenetics is used to describe the heritable changes in gene expression and phenotype without any alterations in the DNA sequence itself (Holliday 1994). Epigenomics means the genome-wide analysis of the epigenetic process (Ideraabdullah and Zeisel 2018). Epigenetics can be broadly classified into three principle mechanisms (Bishop and Ferguson 2015; Virani et al. 2012):

1. Chromatin regulatory processes

2. DNA Methylation

3. Non-coding RNA

In each eukaryotic cell, the DNA strand is around 2 meters in length. To fit itself into a small nucleus of some $6 \mu \mathrm{m}$ diameter, the DNA coils around nuclear proteins called histones and forms a compact structure called chromatin (Hauer and Gasser 2017). In this process, the DNA becomes inaccessible for DNA binding factors and hence does not get transcribed. This state of chromatin which displays a high degree of compaction is defined as heterochromatin. For gene transcription, the chromatin structure needs to shift from a condensed to a transcriptionally accessible relaxed state. This relaxed chromatin state which enables active transcription is termed as euchromatin (Corradini et al. 2007; Fedorova and Zink 2008). The dynamic reorganization of the chromatin architecture and hence the regulation of gene transcription are controlled by two major processes: Chromatin remodeling and histone modification.

Given the critical involvement of chromatin regulatory processes in controlling gene transcription, alterations of chromatin-associated processes contribute to several pathophysiological conditions, including cancer (Bauer and Martin 2017; Morgan and Shilatifard 2015). For example, mutation of chromatin regulatory proteins or their altered activity have a severe impact on cellular functions that support the development and progression of cancer (Jian Chen et al. 2016). Accordingly, in PDAC approximately $38 \%$ of specimens carry mutational events in chromatin regulatory proteins (P. Bailey et al. 2016), which can have prognostic implications (Grassi et al. 2018). Moreover, several genetic alterations in PDAC and subsequent dysregulation in signaling pathways influence on the expression, recruitment and activity of chromatin 


\section{INTRODUCTION}

regulatory proteins with potential impact on the chromatin landscape (Liu et al. 2016).

The following paragraphs summarize the major physiological mechanisms controlling chromatin remodeling and histone modifications and provide examples, how alterations of chromatin regulatory protein functions can contribute to PDAC development and progression.

\subsubsection{Nucleosome remodeling}

The nucleosome is the fundamental unit of chromatin. Each nucleosome is a DNA-protein complex, which comprises 146 bp of DNA wrapped around a histone octamer (2 sets of histone $\mathrm{H} 2 \mathrm{~A}, \mathrm{H} 2 \mathrm{~B}, \mathrm{H} 3$, and H4) (Van Holde et al. 1980). Nucleosome remodeling is mediated by large multiprotein complexes that contain several subunits, including the Adenosine triphosphatase (ATPase) catalytic subunit. The energy released by the hydrolysis of ATP is utilized to slide nucleosome along the DNA, thereby exposing the DNA to transcription factors (Tyagi et al. 2016). Currently, four families of remodeling complexes are known: SWI/SNF, ISWI, CHD and INO80, (Musladin et al. 2014) each with distinct biological functions. All complexes have a conserved ATPase subunit and other members are responsible for the complexes' recruitment to DNA or regulate the ATPase activity (Mani et al. 2017).

The subunits of the complexes are known to be inactivated in many human cancers and are hence referred to as tumor suppressors. Importantly, according to investigations by Bailey et al., mutations in components of the SWI/SNF complex were detected in $14 \%$ of PDAC samples ( $P$. Bailey et al. 2016). Importantly, inactivation of selective SWI/SNF complex members has been associated with poor survival in PDAC patients (Yoon et al. 2019) and impact on the senstitivity towards platinum-based therapy regimens (Hasan and Ahuja 2019), hence emphasizing the prognostic and predictive relevance of these alterations. 


\section{INTRODUCTION}

\subsubsection{Histone modifications}

Histone tails are susceptible to post-translational modifications, generally referred to as 'histone marks'. The type of histone mark and its relative location on the gene (Transcription start site (TSS), promoters, gene body) determines if the gene is expressed or repressed. The enzymes that mark the histones are referred to as writers, readers or erasers, which add, recognize or remove the mark, respectively (Janzen et al. 2010). The consequences of distinct histone modifications on DNA accessibility and transcriptional activity are determined by the type (e.g. acetylation, methylation, phosphorylation, ubiquitination and SUMOylation) and the localization (histone protein and amino acid residue) of the histone modification (Audia and Campbell 2016; Goll and Bestor 2002).

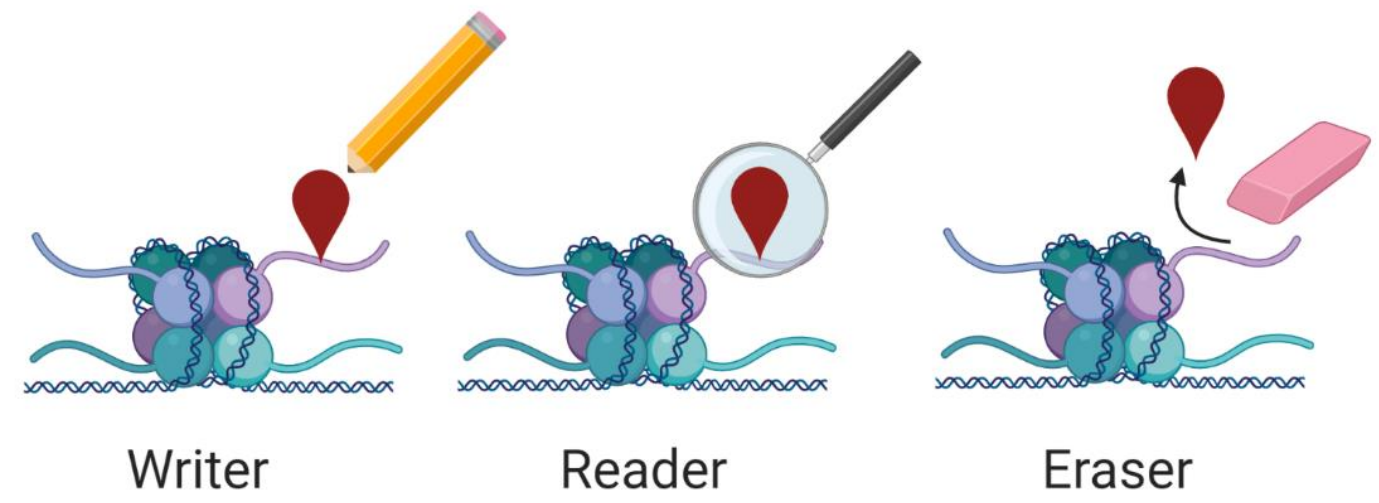

Figure 2: Schematics depicting the chromatin regulators.

Three main categories of chromatin regulators responsible for post-translational modifications (PTMs) include writers (adds the modification), readers (recognizes particular histone marks) and erasers (catalyze the removal of histone modification).

\subsubsection{Histone acetylation}

In the nucleosome, negatively charged DNA and the positively charged histones form the DNA-protein complex (Tammen, Friso, and Choi 2013). Histone acetylation (also known as lysine acetylation) is the process of adding acetyl groups onto histone tail residues. This neutralizes the positive charge on the amino acid residue, thereby loosening DNA-histone contact resulting in an open chromatin structure that enables gene transcription (Sterner and Berger 2000). On the contrary, histone deacetylation, where acetyl groups are erased, leads to compaction of the DNA-histone structure resulting in gene repression 
(De Ruijter et al. 2003). The addition and removal of acetyl groups on lysine residues are mediated by histone acetyltransferases (HATs) and Histone deacetylases (HDACs), respectively (Singh, Reindl, and Jansen 2019). Further, acetylated marks serve as docking sites where chromatin reader proteins bind (Yun et al. 2011).

Aberrant activity of HATs, HDACs and acetylation readers are correlated with PDAC development and progression (Glozak and Seto 2007; Köenig et al. 2010; Ouaïssi et al. 2014; Stenzinger et al. 2013). Considering the implications of aberrant acetylation in cancer, several inhibitors are being developed against histone lysine writers (Kunnumakkara et al. 2007; Sahu, Batra, and Srivastava 2009), readers (Pérez-Salvia and Esteller 2017; Y. Xu and Vakoc 2017) and erasers (Marmorstein and Zhou 2014; Mottamal et al. 2015).

\subsubsection{Histone methylation}

Histone methylation is the process where methyl groups are added to lysine/arginine residues of histones (Hyun et al. 2017). Unlike acetylation which is always associated with gene activation, methylation can be correlated to either gene expression or repression depending on which lysine/arginine residue is methylated and how many methyl groups are added (mono-, di- or trimethylation) (Dambacher, Hahn, and Schotta 2010). For instance, trimethylation of lysine 4 on histone $\mathrm{H} 3$ (H3K4me3) by the trithorax complex induces gene expression whereas H3K27me3 installed by members of the Polycomb repressor complex 2 (PRC2) induces gene repression (Schuettengruber et al. 2007). Histone methyltransferases (HMTs) which methylate lysine and arginine residues on histones are referred to as lysine methyltransferases (KMTs) and protein arginine methyltransferases (PRMTs), respectively. (Lorenzo and Bedford 2011; Upadhyay and Cheng 2011). Around 50 KMTs are known so far, where all of them (except Dot1) have a conserved Su(var)3-9,EZH2 and Trithorax (SET) domain, which is responsible for the methyltransferase activity (G. A. Lomberk, lovanna, and Urrutia 2016). Lysine demethylases (KDMs) counteracts KMT activity by mediating the removal of methyl marks (Thinnes et al. 2014).

Many histone- methylases and demethylases are known to be mutated, dysregulated, overexpressed, or downregulated in various cancers (Singh, 10 


\section{INTRODUCTION}

Reindl, and Jansen 2019). For instance, studies showed that mutations in MLL1, MLL3 and MLL4 and reduced expression of MLL3 and MLL4 had a better prognosis and outcome in PDAC (Dawkins et al. 2016). In contrast, high levels of KDM1A in PDAC is associated with poor survival. Also, several other KDMs (KDM2A, KDM3A, KDM5B) are highly expressed in various cancers (McGrath and Trojer 2015). Histone methyltransferases represent another class of attractive druggable targets for PDAC intervention. BRD-4770, a molecular inhibitor of G9a was found to decrease methylation levels, induce cell cycle arrest and mediate cell senescence (Artal-Martinez de Narvajas et al. 2013; Yuan et al. 2012). Another G9a inhibitor, BIX-01294, also showed similar results in vitro in pancreatic cancer (Cui et al. 2015). One of the early nonselective KDM1 inhibitors, tranylcypromine, is approved by the FDA for neural disorders (Thinnes et al. 2014). Several compounds are being tested for the development of selective KDM inhibitors (G. A. Lomberk, lovanna, and Urrutia 2016).

\subsection{PRC2 complex}

The protein complex families - trithorax group (trxG) genes and polycomb group (PcG) genes were initially discovered in Drosophila Melanogaster, where they regulate the expression of homeotic genes (Hox genes) during the formation of body plan (Deschamps et al. 1999). They work antagonistically with trxG maintaining and PcG silencing transcription processes, respectively (Kennison 1995; Schuettengruber et al. 2007). PcG assembles into two types of multiprotein complexes- Polycomb Repressive Complex 1 (PRC1) and Polycomb Repressive Complex 2 (PRC2) (Sauvageau and Sauvageau 2010). The PRC2 complex mediates H3K27me3 which is then recognized by members of the PRC1 complex, which further mediates H2AK119Ub1. This causes the chromatin to condense, thereby leading to transcriptional silencing. 


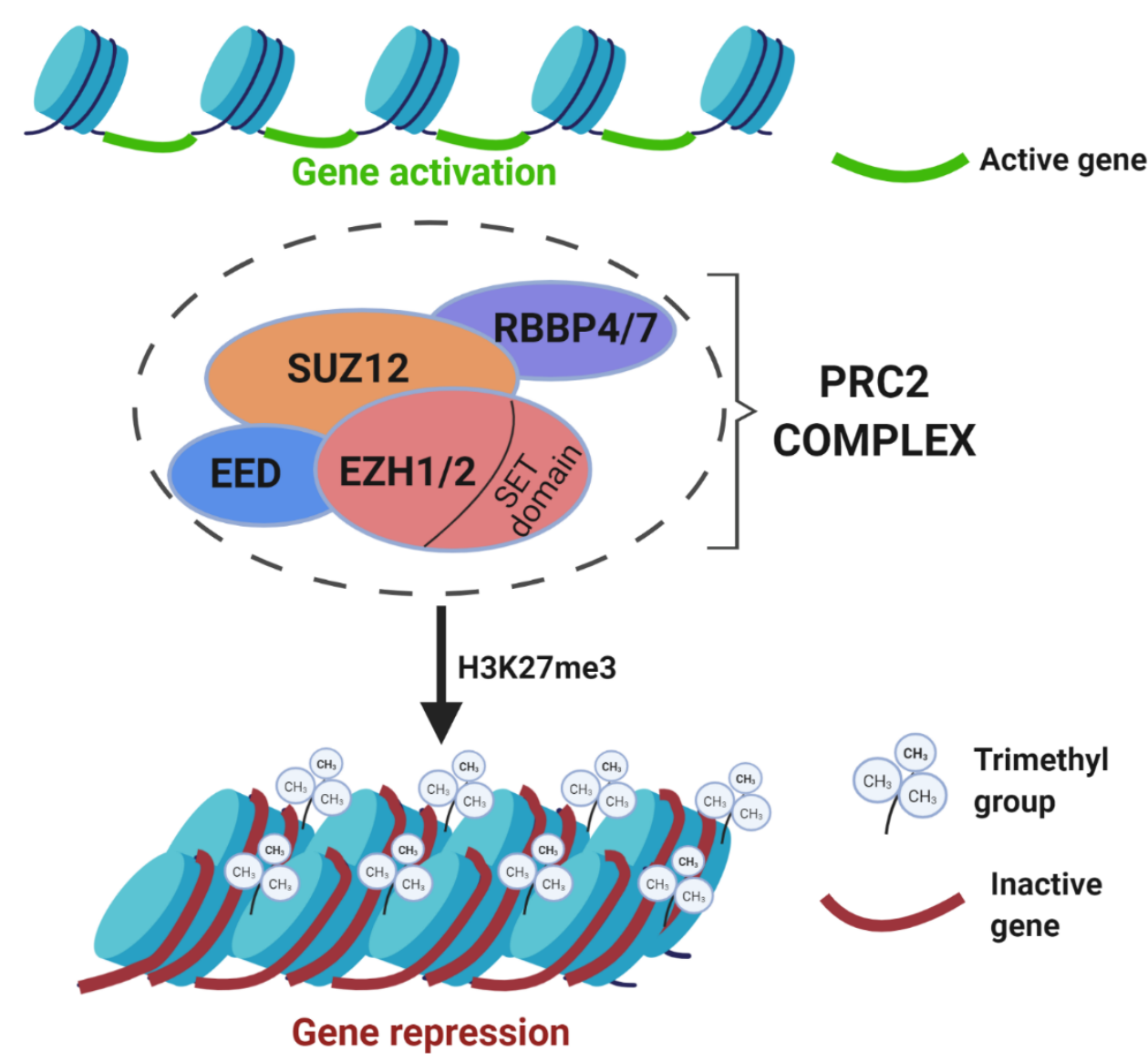

Figure 3: Schematic illustrating the function mediated by the PRC2 complex

The core subunits of the PRC2 complex are Enhancer of Zeste 2 (EZH2), Suppressor of Zeste 12 (SUZ12), Embryonic ectoderm development (EED) and Retinoblastoma binding proteins 4 and 7 (RBBP4/7). The complex mediates addition of three methyl groups on lysine 27 of histone H3 (H3K27me3) subsequently switching the chromatin from an active transcription state to a repressive state.

The PRC2 complex consists of four core members - Enhancer of Zeste 2 (EZH2), Embryonic ectoderm development (EED), Supressor of Zeste 12 (SUZ12) and Retinoblastoma binding proteins 4 and 7 (RBBP4/7) as well as other accessory proteins (AEBP2, PCL, JARID2) (Aranda, Mas, and Di Croce 2015). EZH2 is the SET domain-containing catalytic subunit of the complex which mediates H3K27me3. (Müller et al. 2002) WD40 domain harboring component, EED, recognizes the trimethylated residues and zinc finger containing subunit, SUZ12, maintains the enzymatic activity of EZH2 (van Mierlo et al. 2019). The additional accessory proteins modulate the PRC2 complex (Ketel et al. 2005). Drosophila has a single Ezh gene whereas 


\section{INTRODUCTION}

mammals have two - EZH1 and EZH2 which are paralogs. Even though both $\mathrm{EZH} 1$ and $\mathrm{EZH} 2$ harbor H3K27me3 activity, PRC2-EZH1 shows relatively lower methyltransferase activity than PRC2-EZH2. Also, EZH2 is mostly present in proliferating cells whereas EZH1 is found in dividing cells (Margueron et al. 2008). Genome-wide studies in human embryonic fibroblast cells show that PRC2 and H3K27 methylation mark co-occupy on several differentiationassociated genes (Bracken et al. 2006).

\subsubsection{Regulation of EZH2 expression in cancer}

EZH2 is an extensively studied SET domain-containing histone methyltransferase, which silences gene expression by catalyzing trimethylation of H3K27 (Chou et al. 2015). EZH2 dysregulation is frequently seen in a wide variety of cancers. Altered EZH2 expression and activity can be caused by activating/inactivating mutations of $\mathrm{EZH} 2$ which are primarily found in hematological malignancies (Jankowska et al. 2011; Morin et al. 2010) or by missense mutation (K27M) in H3.3 which is found in pediatric gliomas (KhuongQuang et al. 2012). Besides these genetic events targeting $E Z H 2$, in solid tumors the histone methyltransferase is mostly overexpressed. Overexpression of $\mathrm{EZH} 2$ can also be caused by various transcriptional pathways. For example, the Mitogen-activated protein kinase kinase (MEK) - extracellular signalregulated kinase (ERK) - ETS Like-1 (ELK1) pathway has been shown to be responsible for increased EZH2 expression in ERBB2 overexpressing and triple-negative breast cancers. Phosphorylated ELK1 binds to its binding motif on the EZH2 promoter thereby activating EZH2 transcription (Fujii et al. 2011). Another pathway involved in EZH2 regulation is Retiniblastoma protein ( $p R b)$ E2F signaling. Upon phosphorylation of $p R b$, E2F dissociates from the $p R b$ E2F complex, binds to its binding site on the EZH2 promoter thereby promoting EZH2 transcription (Margueron et al. 2008). Abberation of the pRb-E2F pathway or E2F overexpression is associated with $\mathrm{EZH} 2$ overactivation in breast and bladder tumors (Feber et al. 2004; Margueron et al. 2008). Cancerrelated transcription factors bind on the $E Z H 2$ promoter and activate its mRNA expression. In breast cancer, hypoxia inducible factor 1a (HIF1a) induced by the hypoxic environment binds to its consensus sequence on $E Z H 2$ and activates its expression thereby aggravating breast cancer (Mahara et al. 
2016). Besides, miRNAs downregulate $E Z H 2$ levels by post-translational modifications. Many different miRNA s like miR-25, -98, -124, -138, -214 interact with specific sequences in the EZH2 3' untranslated region (3' UTR) (Völkel et al. 2015). Together, aberrant EZH2 expression in cancer can be caused by various mechanisms which occur at different regulatory levels.

\subsubsection{Context-dependent roles of EZH2}

EZH2 overexpression was initially detected in breast and prostate cancer through microarray analysis where it was strongly correlated with poor prognosis and associated with high-grade metastatic stages (Bachmann et al. 2006; Varambally et al. 2002). High levels of EZH2 are reported in a wide variety of other cancers like melanoma (Fan et al. 2012; Zingg et al. 2015), lung (Hussain et al. 2009), hepatocellular (Sudo et al. 2005), bladder (Raman et al. 2005), ovarian (Moses and Jia 2013; Rao et al. 2010), brain (Bracken et al. 2003) and pancreatic cancer (Han et al. 2016; Ougolkov, Bilim, and Billadeau 2008; Toll et al. 2010). In these solid tumor entities EZH2 mainly serves as an oncogenic factor by silencing tumor suppressor genes via H3K27me3-mediated transcriptional repression. However, apart from its PRC2-dependent activity, EZH2 exhibits various other modes of action depending on the cellular context. For instance, EZH2 mediates posttranslational methylation of many non-histone proteins like GATA4 (He et al. 2012), RORa (Lee et al. 2012) and TALIN (Gunawan et al. 2015). In addition to mediating gene repression, EZH2 also functions in a PRC2 independent manner to mediate transcriptional activation. In the estrogen receptor-positive luminal-like breast cancer cell line MCF7 for example, EZH2 mediates CYCLIND1 and MYC transcription independent of its methyltransferase activity (Shi et al. 2007). Another instance where EZH2 functions as an activator is in castration-resistant prostate cancer. EZH2 acts via its methyltransferase activity but without relying on other PRC2 complex members. In this case, EZH2 gets phosphorylated posttranslationally at serine 21 , and then biochemically interacts with the androgen receptor to work as a transcriptional coactivator activating downstream target genes ( $K . \mathrm{Xu}$ et al. 2012).

While the aforementioned examples highlight the oncogenic activity of EZH2, there is also evidence for tumor suppressive functions of the histone 


\section{INTRODUCTION}

methyltransferase. For instance, Ntziachristos et al. found that loss-of-function EZH2 mutations promote tumor progression in T-cell acute lymphoblastic leukemia (T-ALL) (Ntziachristos et al. 2012). In another study, Bremer et al. demonstrated that high EZH2 expression significantly correlated with favorable prognosis in colorectal cancer (CRC) patients (Bremer et al. 2019). Another example is in renal cell carcinoma where loss of EZH2 mediates HIFdependent CXCR4 activation consequently promoting cancer metastasis (Vanharanta et al. 2013).

Context-dependent EZH2 activities have also been described in the pancreas where $\mathrm{EZH} 2$ is critically involved in the regulation of cellular plasticity. Accordingly, EZH2 has been described as a critical regulator of acinar cell regeneration following injury. Mallen-St. Clair et al. showed that EZH2 represses CDKN2A (encoding for the negative cell cycle regulator $\mathrm{p} 16$ ), thus allowing the proliferation of ADM which is a prerequisite for acinar redifferentiation (Mallen-St. Clair et al. 2012). Further mechanistic analyses suggest that EZH2-driven pancreatic regeneration critically involves transcriptional repression of the NFATC1 gene (N. M. Chen et al. 2017). The inflammatory transcription factor Nuclear factor of activated T cells (NFATc1) is activated upon acinar cell injury and blocks pancreatic regeneration. In KRAS wildtype cells, EZH2 counteracts NFATc1 activity by binding to the TSS of the NFATC1 gene and transcriptionally repressing its expression, which then allows the redifferentiation of acinar cells in later stages of pancreatic recovery. However, the EZH2-NFATc1 axis does not operate in the same way in the context of PDAC. In the presence of oncogenic KRAS, EZH2 transcriptionally activates NFATC1 expression, which further leads to ADM formation and progression into PDAC. Hence, KRAS acts as a switch that regulates opposing roles of EZH2 in acinar cell regeneration vs. transformation (N. M. Chen et al. 2017).

Overall, EZH2 activity are highly context dependent. While EZH2 is crucial during development and regeneration of the organ, it shifts its role in cancer. It functions via various modes of action, promotes either oncogenic or tumorsuppressive implications and is regulated at different levels by several mechanisms. 
INTRODUCTION

\subsubsection{EZH2 in PDAC}

EZH2 was identified as an oncogenic driver in PDAC by Ougolvou and colleagues, where they found nuclear overexpression of the histone methyltransferase in $68 \%$ of PDAC patients. They elegantly provide functional insights into the role of $\mathrm{EZH} 2$ in mediating cell proliferation and chemoresistance. Their study also reported that EZH2 silencing induced apoptosis and increased the sensitivity towards gemcitabine and doxorubicin (Ougolkov, Bilim, and Billadeau 2008). Another report in 2012 by Toll et al. supported this study and reported that EZH2 depletion sensitizes pancreatic cancer cells to gemcitabine and subsequently induces apoptosis. They evaluated $E Z H 2$ levels in PDAC samples from patients treated with gemcitabine. Patients with low EZH2 levels survived for a significantly longer time compared to the ones with high EZH2 levels. This validates the correlation between high EZH2 levels and low gemcitabine response. Furthermore, their study was the first to show that high $\mathrm{EZH} 2$ levels are associated with reduced E-cadherin levels in PDAC (Toll et al. 2010). The EZH2-E-cadherin axis was further validated by Han et al., where they additionally showed that EZH2 mediates cell invasion and migration by transcriptionally repressing $C D H-1$. In their study, patients harboring an abundance of EZH2 and low E-cadherin displayed lower survival (Han et al. 2016). Altogether, EZH2 is highly expressed and harbors oncogenic activity in pancreatic cancer.

\subsubsection{EZH2 inhibitors}

Considering that H3K27me3 is one of the frequently dysregulated histone modifications in a lot of cancers including PDAC, and EZH2 is the mediator for depositing this mark, it has been a major target for the development of inhibitors. One of the first inhibitors developed for EZH2 inhibition was 3deazaneplanocin (DNZep), an S-adenosyl-L-homocysteine (SAH) hydrolase inhibitor, which increases $\mathrm{SAH}$ levels, thereby leading to inactivation of several methyltransferases including EZH2. It showed promising activity in breast cancer and prostate cancer diminishing EZH2 and H3K27me3 levels and restoring downstream target genes of PRC2 (Tan et al. 2007). This small molecule inhibitor also reduced the self renewal capacity of prostate, ovarian and glioblastoma stem cells (Völkel et al. 2015). One of the studies reported 


\section{INTRODUCTION}

that DNZnep boosted the antiproliferative effect of gemcitabine in PDAC cell lines and primary cultures derived from PDAC tumors. Despite this, the use of this drug has been terminated due to its high toxicity levels (Gaudichon et al. 2014).

Following this up, S-adenosyl-L-methionine (SAM) competitive EZH2 inhibitors like EPZ005687, GSK2816126, CPI-1205, UNC1999 and EPZ6438 (tazemetostat) were discovered through high throughput screening approaches. They display 1000 times more selectivity for EZH2 over other methyltransferases and can effectively antagonize EZH2 activity in the nanomolar range (McGrath and Trojer 2015). GSK126 was effective in monolayers in gastric cancer and lung adenocarcinoma cell lines as well as in xenografts models of lymphoma, where it displayed a decrease in cell proliferation, angiogenesis, and a significant increase in apoptosis (Y. T. Chen et al. 2016; McCabe et al. 2012). The compound CPI-1205 exhibited a decrease in EZH2 and H3K27me3 levels. Additionally, it displayed antiproliferative effects and led to increased apoptosis in vitro in medulloblastoma. It is relatively well tolerated and currently in clinical trials for medulloblastoma and B cell lymphoma (Miele et al. 2017; Vaswani et al. 2016). UNC1999, an orally bioavailable EZH2 inhibitor induced durable tumor response successfully in monolayers, spheroid cultures and pdx mouse models of PDAC (Huang et al. 2015b). Tazematostat (EPZ6438) is a first in class, highly selective, orally administered EZH2 inhibitor that has been tested in a phase-I study. It exhibited beneficial results with a favorable safety profile and anti-tumor activity in patients with $B$ cell non-Hodgkin lymphoma and SMARCA4-negative or INI1 negative advanced solid tumors (Italiano et al. 2018; Richart and Margueron 2020). Moreover, a disease control rate of $55 \%$ was observed in solid tumors with this epidrug. Currently, Tazematostat is in phase-Il clinical trials (NCT01897571) (Hessmann et al. 2017). To date, three EZH2 inhibitors, CPI-1205, GSK2816126 and tazematostat (EPZ 6438) have shown considerable tolerance and efficiency in hematological malignancies and solid cancers. 


\section{INTRODUCTION}
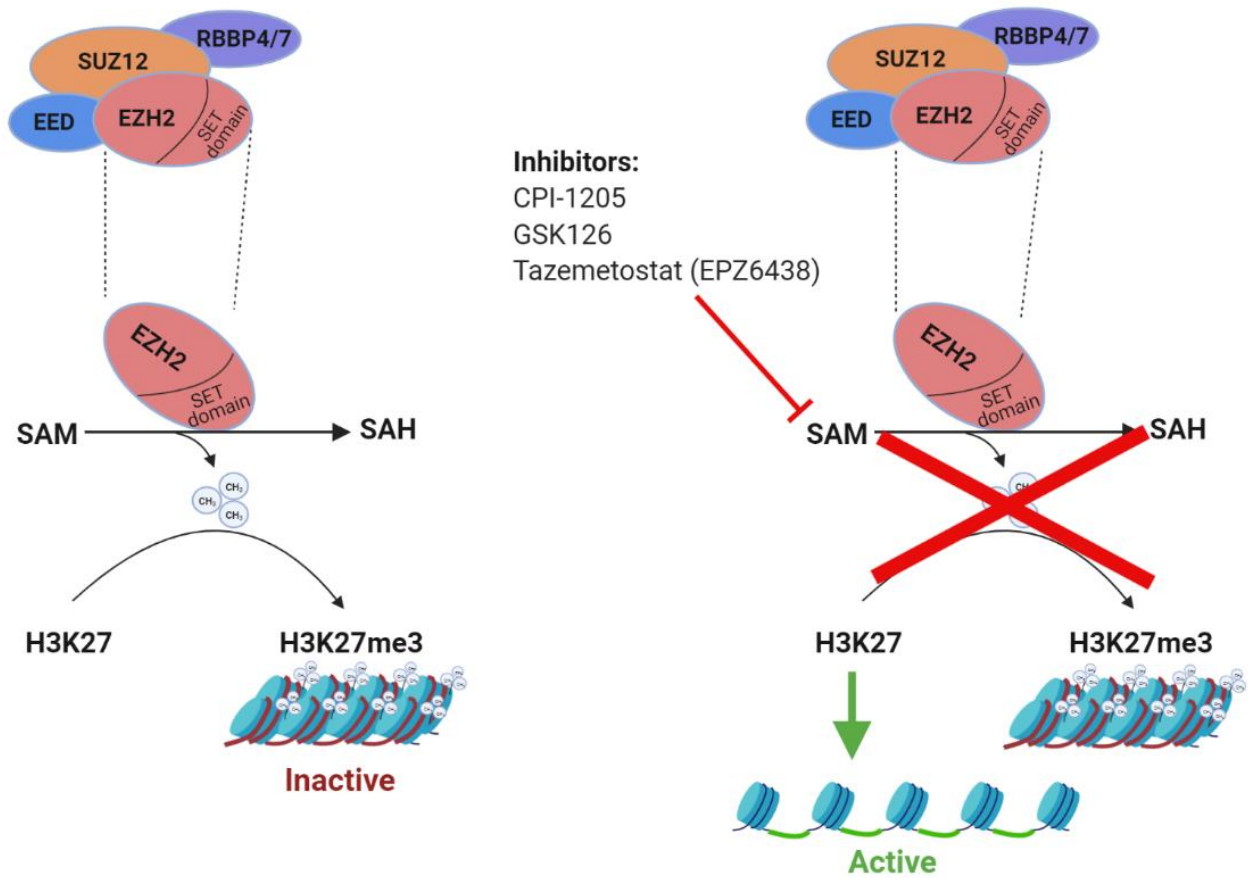

Figure 4: EZH2 SET-domain inhibitors and their mechanism of action.

The catalytic unit of the PRC2 complex, EZH2, harbours a Su(var)3-9,EZH2 and Trithorax (SET) domain which catalyzes the transfer of methyl groups from the methyl donor S-Adenosyl-L-methionine (SAM) to H3K27, thus leading to transcriptional repression. The competitive SAM inhibitors (belonging to a class of EZH2 inhibitors) block the methylation process, thus keeping the chromatin in an active transcriptional state. (Adapted from (Gulati, Béguelin, and Giulino-Roth 2018)).

Although EZH2 has a more prominent role in mediating H3K27me3, EZH1 contributes to maintaining the methylation levels. Hence, studies suggest that dual inhibitors like DS-3201, which simultaneously inhibit both EZH1 and EZH2, are more effective (Honma et al. 2017). The same report also argues that a combination of El1 and EED-226, which are EZH2 and EED inhibitors, respectively increased the treatment efficacy compared to monotherapies. Most recently, an EZH2 degrader has been characterized which efficiently degrades $\mathrm{EZH} 2$ protein making this compound highly specific for EZH2. Furthermore, this is a valuable epidrug for cancers where EZH2 plays a methyltransferase independent role ( $\mathrm{Ma}$ et al. 2020).

The majority of these small molecule inhibitors of EZH2 exhibit an optimistic effect as monotherapy in preclinical models of PDAC (Huang et al. 2015a; 


\section{INTRODUCTION}

Paradise, Barham, and Fernandez-Zapico 2018). But the combination of these inhibitors with a chemotherapeutic agent or another epidrug seems to work better in PDAC than a monotherapy (Honma et al. 2017; Ougolkov, Bilim, and Billadeau 2008). Further exploration of these inhibitors in larger clinical trials as targeted therapy is necessary for maximizing its benefits for translational utility. Furthermore, given that EZH2 exhibits a highly context-dependent expression and function, inhibiting EZH2 might not be benefical in all situations or might even foster cancer progression. Indeed, some studies demonstrate that EZH2 inhibitors were only effective in subgroups of cancer patients. For instance, Puppe et al. showed in 2009 that EZH2 inhibition by DZNep was selectively beneficial in BRCA-1 deficient or mutated breast cancer cells as these cells promoted tumor progression in an EZH2 dependent manner whereas BRCA-1 proficient cells were not affected by EZH2 inhibition (Puppe et al. 2009). In certain subsets of breast cancer (Shi et al. 2007), EZH2 mediates tumor progression in a methyltransferase independent manner and in such cases inhibitors blocking the SET domain of EZH2 would not be efficacious while inhibitors that degrade EZH2 protein would potentially be more effective (Ma et al. 2020). Besides, another study suggests that while inhibiting EZH2 in cancers harboring EZH2 Gain-of-function (GOF) mutations like in follicular lymphomas ( $F L$ ) and Diffuse large $B$ cell lymphomas (DLBCL) is very effective, the inhibitors were not useful in cells harboring EZH2 Loss-of-function (LOF) mutations like in certain cases of Myeloproliferative neoplasms (MPN) and Tcell acute lymphoblastic leukemia (T-ALL) (Honma et al. 2017). Further several lines of evidence demonstrate that blocking EZH2 is specifically impactful in cancer cells harboring mutation or loss of SWI/SNF subunits - in lung cancer cells (K. H. Kim et al. 2015), small cell carcinoma of ovary (Chan-Penebre et al. 2017) and other rhaboid tumors (Knutson et al. 2013; Wilson et al. 2010). These studies argue that it is necessary to understand the molecular conditions underlying EZH2 mediated tumor progression in cancer before designing strategies to inhibit it. 


\section{INTRODUCTION}

\subsection{Aims of the study}

EZH2 consitutes a widely studied epigenetic factor in development as well as in cancer. Its implications in various cancer types including PDAC characterize the histone methyltransferase as a promising therapeutic target in PDAC. However, recent findings also suggest that the activity, target gene selection and regulation of chromatin regulatory proteins like EZH2 occur in a highly context-dependent manner. Hence, the successful application of EZH2 inhibition in PDAC treatment requires an in-depth understanding of how the histone methyltransferase promotes PDAC progression and depends on the elucidation of the molecular dependencies determining the efficacy of EZH2 inhibition in PDAC.

In this study, we aim at investigating the role of EZH2 in pancreatic cancer progression and plasticity and aim at gaining mechanistic insights into EZH2dependent gene regulation in PDAC. Our specific aims are:

1) To dissect the functional implications of $E Z H 2$ in PDAC development and progression.

2) To identify EZH2-dependent gene signatures in PDAC progression.

3) To elucidate the molecular prerequisites determining the efficacy of EZH2 targeting in PDAC. 


\section{MATERIALS}

\subsection{Equipments:}

\begin{tabular}{|l|l|}
\hline EQUIPMENT & COMPANY \\
\hline Agarose electrophoresis chamber & $\begin{array}{l}\text { Peqlab Biotechnologie GmbH, } \\
\text { Erlangen, Germany }\end{array}$ \\
\hline Arium ${ }^{\circledR}$ pro ultrapure water system & Sartorius, Göttingen, Germany \\
\hline Aspirator with trap flask & $\begin{array}{l}\text { Grant Instruments Ltd, Cambs, } \\
\text { England }\end{array}$ \\
\hline Bioanalyzer ( 2100, 5067-4626) & Agilent technologies, USA \\
\hline Biorupter® Pico & Diagenode, Liege, Belgium \\
\hline Class II safety cabinet (S2020 -1.2) & $\begin{array}{l}\text { Thermo Fisher Scientific, Waltham, } \\
\text { USA }\end{array}$ \\
\hline Cold plate (Histocore Arcadia c) & Leica Biosystem, Wetzlar, Germany \\
\hline Dri-Block Heater DB2A & Techne, Staffordshire, UK \\
\hline Fluorescence Microscope System & Leica Camera, Wetzlar, Germany \\
\hline gentleMACS dissociator & Miltenyi Biotech, Germany \\
\hline Heating plate & Leica Biosystem, Wetzlar, Germany \\
\hline HERAcell 240i CO incubator & Thermo Scientific, Waltham, USA \\
\hline Ice flaker (AF80) & Scotsman, Edinburgh, UK \\
\hline INTAS- ECL Chemostar imager & $\begin{array}{l}\text { INTAS Science Imaging Instruments } \\
\text { GmbH, Göttingen, Germany }\end{array}$ \\
\hline Inverted microscope (CKX53SF) & Olympus, Tokyo, Japan \\
\hline Light microscope "BX43" & Olympus, Tokyo, Japan \\
\hline Magnetic stirrer (RH B S000) & IKA ${ }^{\circledR}$ Laboratory equipment, Germany \\
\hline Microplate Luminometer "LUMO" & $\begin{array}{l}\text { Autobiolabtec Instruments Co.,Ltd, } \\
\text { China }\end{array}$ \\
\hline Microplate reader "PHOmo" & $\begin{array}{l}\text { Autobiolabtec Instruments Co.,Ltd, } \\
\text { China }\end{array}$ \\
\hline Microwave (NN-E209W) & Panasonic; Japan \\
\hline Microtome (Leica RM2265) & Leica Biosystems, Wetzlar, Germany \\
\hline Mini-PROTEAN Tetra Cell & Bio Rad Laboratories, Hercules, USA \\
\hline MSA Minishaker & IKA, Staufen, Germany \\
\hline Multifuge X1 Centrifuge Series & $\begin{array}{l}\text { Thermo Fisher Scientific, Waltham, } \\
\text { USA }\end{array}$ \\
\hline
\end{tabular}




\begin{tabular}{|l|l|}
\hline EQUIPMENT & COMPANY \\
\hline Multipette plus & Eppendorf, Hamburg, Germany \\
\hline NanoPhotometer P-330 & $\begin{array}{l}\text { Intas Science Imaging Instruments, } \\
\text { Goettingen, Germany }\end{array}$ \\
\hline Neubauer chamber & Assistant, Sondheim/Rhön, Germany \\
\hline Paraffin Tissue embedder (EG1150H) & Leica Biosystems, Wetzlar, Germany \\
\hline pH meter (FiveEasy & $\begin{array}{l}\text { MM Plus FEP20) } \\
\text { Switzerland }\end{array}$ \\
\hline PerfectSpin 24R Refrigerated & Peqlab, Erlangen, Germany \\
\hline Microcentrifuge & $\begin{array}{l}\text { INTEGRA Biosciences, Biebertal, } \\
\text { Germany }\end{array}$ \\
\hline Powerboy acu 2 & Bio Rad Laboratories, Hercules, USA \\
\hline Precision balance PCB & Kern \& Sohn, Balingen, Germany \\
\hline PSU-20i Orbital Shaking Platform & Grant Instruments, Shepreth, UK \\
\hline Refrigerator 4C (Comfort) & Liebherr, Bulle, Switzerland \\
\hline $\begin{array}{l}\text { Safe 2020 Class II Biological Safety } \\
\text { Cabinets }\end{array}$ & $\begin{array}{l}\text { Thermo Fisher Scientific, Waltham, } \\
\text { USA }\end{array}$ \\
\hline $\begin{array}{l}\text { Sequenza } \\
\text { system) } \text { slide rack(cadenza }\end{array}$ & TED PELLA,INC, Redding, CA \\
\hline $\begin{array}{l}\text { Shandon coverplate } \\
\text { system) (cadenza }\end{array}$ & Thermo Scientific, Waltham, USA \\
\hline Sprout Minicentrifuge & $\begin{array}{l}\text { Biozym Scientific, Hessich Oldendorf, } \\
\text { Germany }\end{array}$ \\
\hline StepOnePlus Real-Time PCR System & $\begin{array}{l}\text { Thermo Fisher Scientific, Waltham, } \\
\text { USA }\end{array}$ \\
\hline Thermomixer 5436 & Eppendorf, Hamburg, Germany \\
\hline Tissue dehydration machine & Leica Biosystems, Wetzlar, Germany \\
\hline Trans-Blot Turbo Transfer System & Bio Rad Laboratories, Hercules, USA \\
\hline $\begin{array}{l}\text { TX-400 4 x 400mL Swinging Bucket } \\
\text { Rotor }\end{array}$ & $\begin{array}{l}\text { Thermo Fisher Scientific, Waltham, } \\
\text { USA }\end{array}$ \\
\hline Universal Oven UN55 & Memmert, Schwabach, Germany \\
\hline VacuuHandControl VHCpro & Vacuumbrand, Wertheim, Germany \\
\hline Vacuum pump: BVC Control & Vacuumbrand, Wertheim, Germany \\
\hline Waterbath (WNB14) & Sartorius AG, Göttingen, Germany \\
\hline Weighing balance & \\
\hline
\end{tabular}




\begin{tabular}{|l|l|}
\hline EQUIPMENT & COMPANY \\
\hline Qubit (Q32854) Fluorometer & Invitrogen GmbH, Karlsruhe \\
\hline$-20^{\circ} \mathrm{C}$ freezer & Liebherr, Bulle, Switzerland \\
\hline $\begin{array}{l}-80^{\circ} \mathrm{C} \text { Ultra low temperature freezer } \\
\text { (MDF-U54V) }\end{array}$ & Sanyo electric Co.,Ltd Japan \\
\hline
\end{tabular}

\subsection{Consumable materials}

\begin{tabular}{|c|c|}
\hline MATERIALS & COMPANY \\
\hline Centrifuge tube 15 and $50 \mathrm{ml}$ & Sarstedt, Nümbrecht, Germany \\
\hline $\begin{array}{l}\text { Cell culture Inserts - 8um pore size } \\
\text { (BD 353097) }\end{array}$ & Falcon, Germany \\
\hline Cell strainer-100 $\mu \mathrm{m}$ & Falcon, Durham, USA \\
\hline Cell scrapper & Sarstedt, Nümbrecht, Germany \\
\hline Cello meter counting chamber & Nexcelom Bioscience, Lawrence, MA \\
\hline Cryo pure vial & Sarstedt, Nümbrecht, Germany \\
\hline Combitips advanced (Multipipette tips) & $\begin{array}{l}\text { Starlab International GmbH, Hamburg, } \\
\text { Germany }\end{array}$ \\
\hline $\begin{array}{l}\text { Graduated sterile pipettes }(2,10,25 \\
\text { and } 50 \mathrm{ml})\end{array}$ & $\begin{array}{l}\text { Greiner bio-one, Frickenhausen, } \\
\text { Germany }\end{array}$ \\
\hline Glass coverslips $(24 \times 32,24 \times 60)$ & $\begin{array}{l}\text { Menzel Gläser }{ }^{\circledR} \text {, Menzel GmbH+Co } \\
\text { KG, Braunschweig, Germany }\end{array}$ \\
\hline Micropipette filter tips & $\begin{array}{l}\text { Starlab International GmbH, Hamburg, } \\
\text { Germany }\end{array}$ \\
\hline Micropipette tips & $\begin{array}{l}\text { Starlab International } \mathrm{GmbH} \text {, Hamburg, } \\
\text { Germany }\end{array}$ \\
\hline Microtome blade (S35) & $\begin{array}{l}\text { Feather safety Razer Co. Ltd, Osaka, } \\
\text { Japan }\end{array}$ \\
\hline Microtube (1.5 and $2 \mathrm{ml})$ & Sarstedt, Nümbrecht, Germany \\
\hline Injection needle (Sterile) & B.Braun, Melsungen, Germany \\
\hline Nitrocellulose membrane & $\begin{array}{l}\text { GE Healthcare lifesciences, } \\
\text { Marlborough, USA }\end{array}$ \\
\hline Parafilm & $\begin{array}{l}\text { Pechiney plastic packaging, Chicago, } \\
\text { USA }\end{array}$ \\
\hline PCR tubes & Sarstedt, Nümbrecht, Germany \\
\hline Scalpel & $\begin{array}{l}\text { Feather safety Razer Co. Ltd, Osaka, } \\
\text { Japan }\end{array}$ \\
\hline Serological pipette (2ml) & $\begin{array}{l}\text { Greiner bio-one, Frickenhausen, } \\
\text { Germany }\end{array}$ \\
\hline
\end{tabular}




\begin{tabular}{|l|l|}
\hline MATERIALS & COMPANY \\
\hline Sponge Pad For XCell IITM Blotting & $\begin{array}{l}\text { Novex by Life technologies, Carlsbad, } \\
\text { CA }\end{array}$ \\
\hline Superfrost glass slides (Histology) & Thermo Scientific, Waltham, USA \\
\hline Syringe (1ml) & BD Plastic, Madrid, Spain \\
\hline Syringe (5,10,20ml) & B.Braun, Melsungen, Germany \\
\hline Sterile syringe Filter $(0.2 \mu \mathrm{m})$ & $\begin{array}{l}\text { Labsolute, Th.Geyer ingredients } \\
\text { GmbH \& Co.KG, Höxter, Germany }\end{array}$ \\
\hline Tissue culture multiwell plates & $\begin{array}{l}\text { Greiner bio-one, Frickenhausen, } \\
\text { Germany }\end{array}$ \\
\hline Tissue culture flasks & $\begin{array}{l}\text { Greiner bio-one, Frickenhausen, } \\
\text { Germany }\end{array}$ \\
\hline Tissue culture dishes $(2 \mathrm{~cm}, 10 \mathrm{~cm})$ & Sarstedt, Nümbrecht, Germany \\
\hline Tissue cassette & Sanowa, Leimen, Germany \\
\hline
\end{tabular}

\subsection{Chemicals}

\begin{tabular}{|l|l|}
\hline CHEMICAL & COMPANY \\
\hline Albumin standard & Thermo Scientific, Waltham, USA \\
\hline Agarose & Biozym Scientific GmbH, Oldendorf, Germany \\
\hline Aqua & B.Braun, Melsungen, Germany \\
\hline Bovine serum albumin (BSA) & Serva, Heidelberg, Germany \\
\hline B-Mercaptoethanol & Merck, Darmstadt, Germany \\
\hline Citric acid monohydrate & Carl Roth GmbH Co. KG, Karlsruhe, Germany \\
\hline EDTA & Acros organics, Geel, Belgium \\
\hline EGTA & Sigma-Aldrich, St. Louis, USA \\
\hline Ethanol & $\begin{array}{l}\text { ChemSolute } \\
\text { Co. Th.Geyer ingredients GmbH \& }\end{array}$ \\
\hline Eosin & Sigma-Aldrich, Germany \\
\hline Formaldehyde (4\%) & Merck, Darmstadt, Germany \\
\hline HEPES & Carl Roth GmbH Co. KG, Karlsruhe, Germany \\
\hline Glycerol & Carl Roth GmbH Co. KG, Karlsruhe, Germany \\
\hline Hematoxylin & Sigma-Aldrich, St. Louis, USA \\
\hline Hydrochloric acid & Carl Roth GmbH Co. KG, Karlsruhe, Germany \\
\hline Hydrogen peroxide & Carl Roth GmbH Co. KG, Karlsruhe, Germany \\
\hline
\end{tabular}


MATERIALS

\begin{tabular}{|c|c|}
\hline CHEMICAL & COMPANY \\
\hline Isofluran & $\begin{array}{l}\text { AbbVie Deutschland GmbH \& Co. KG, } \\
\text { Ludwigshafen, Germany }\end{array}$ \\
\hline Methanol & Carl Roth GmbH Co. KG, Karlsruhe, Germany \\
\hline Non-fat milk powder & Carl Roth GmbH Co. KG, Karlsruhe, Germany \\
\hline PBS (Dulbecco's) & Biochrom, Berlin, Germany \\
\hline PMSF & Sigma-Aldrich, St. Louis, USA \\
\hline Roticlear & Carl Roth GmbH Co. KG, Karlsruhe, Germany \\
\hline Rotimount & Carl Roth GmbH Co. KG, Karlsruhe, Germany \\
\hline Saline $(0,9 \% \mathrm{NaCl})$ & B.Braun, Melsungen, Germany \\
\hline Sodium chloride & Merck, Darmstadt, Germany \\
\hline Sodium citrate & Sigma-Aldrich, St. Louis, USA \\
\hline Sodium hydroxide & Acros organics, Geel, Belgium \\
\hline Sodium pyrophosphate & Sigma-Aldrich, St. Louis, USA \\
\hline Sodium pyruvate & Gibco ${ }^{\circledR}$ Thermo scientific, Waltham, USA \\
\hline Tris-HCl & Carl Roth GmbH Co. KG, Karlsruhe, Germany \\
\hline Tris- base & Carl Roth GmbH Co. KG, Karlsruhe, Germany \\
\hline Triton $\mathrm{X}-100$ & Sigma-Aldrich, St. Louis, USA \\
\hline TRIzol & QIAGEN GmbH, Germany \\
\hline Tween-20 & Sigma-Aldrich, St. Louis, USA \\
\hline
\end{tabular}

\subsection{Kits, reagents and inhibitors}

\begin{tabular}{|l|l|}
\hline KITS/REAGENTS/INHIBITORS & COMPANY \\
\hline Agarose A Beads & Merck Millipore, Billerica, USA \\
\hline Agarose G Beads & Merck Millipore, Billerica, USA \\
\hline iScript cDNA Synthesis Kit & Bio Rad Laboratories, Hercules, USA \\
\hline iTaq Universal SYBR Green Supermix & Bio Rad Laboratories, Hercules, USA \\
\hline Midori green & Nippon genetics Europe GmbH \\
\hline Bradford reagent & Bio Rad Laboratories, Hercules, USA \\
\hline Western Lightning ECL/ ECL Ultra & Perkin Elmer, USA \\
\hline Lipofectamine 2000 & Invitrogen, USA \\
\hline siLentFect Lipid reagent & Bio Rad Laboratories, Hercules, USA \\
\hline
\end{tabular}




\section{MATERIALS}

\begin{tabular}{|l|l|}
\hline KITS/REAGENTS/INHIBITORS & COMPANY \\
\hline MycoAlert Mycoplasma Detection Kit & Lonza Group, Basel, Switzerland \\
\hline MicroPlex Library Preparation Kit & Diagenode, Liege, Belgium \\
\hline True seq RNA Library Preparation Kit & Illumina, USA \\
\hline $\begin{array}{l}\text { Trans-Blot Turbo RTA Midi } \\
\text { Nitrocellulose Transfer Kit }\end{array}$ & Bio Rad Laboratories, Hercules, USA \\
\hline $\begin{array}{l}\text { Peroxidase Rabbit/Mouse IgG } \\
\text { Vectastain ABC kits }\end{array}$ & Biozol GmbH, Germany \\
\hline BrdU cell proliferation kit & Roche, 11647229001, Germany \\
\hline cOmplete ${ }^{\text {M }}$ protease inhibitor cocktail & Roche, 11697498001, Germany \\
\hline Tazemetostat/EPZ6438 & ChemieTek, USA \\
\hline DAB ImmPACT & VECTOR Laboratories LTD., UK \\
\hline Matrigel GF R Red/F 10 ml & $\begin{array}{l}\text { Thermo Geyer GmbH\&Co KG, } \\
\text { Germany }\end{array}$ \\
\hline DAPI & Sigma-Aldrich, St. Louis, USA \\
\hline Ponceau solution & Sigma-Aldrich, St. Louis, USA \\
\hline ImmuMount & Thermofisher, Waltham, USA \\
\hline
\end{tabular}

\subsection{Nucleic acids}

\subsection{1 gRNA sequences and validation primers for CRISPR/Cas9-mediated}

EZH2 knockout

\begin{tabular}{|c|c|c|}
\hline & Murine Ezh2 & Human EZH2 \\
\hline $\begin{array}{l}\text { gRNA } \\
\text { sequenc } \\
\text { e }\end{array}$ & $\begin{array}{l}\text { GTGGTGGATGCAACCCGAA } \\
\text { A }\end{array}$ & GTGGTGGATGCAACCCGCAA \\
\hline $\begin{array}{l}\text { Primers } \\
\text { for } \\
\text { knockout } \\
\text { validation }\end{array}$ & $\begin{array}{l}\text { FP: } \\
\text { CCTGTGTAAGTGGGTGTGC } \\
T \\
\text { RP: } \\
\text { GTTTGCTGTCACTTGGCTG } \\
\text { G }\end{array}$ & $\begin{array}{l}\text { FP: } \\
\text { TGCCTATTCGTGATGTTTGGAA } \\
\text { G } \\
\text { RP: } \\
\text { TGTCAACAGCAGGGTGAGAAA }\end{array}$ \\
\hline
\end{tabular}




\subsection{2 siRNA/shRNA oligonucleotides}

\begin{tabular}{|l|l|l|}
\hline siRNA/shRNA & TARGET SEQUENCE (5-3) & SOURCE \\
\hline siEZH2 \#1 & GGAUACAGCCUGUGCACAUTT & Ambion,Carlsbad,USA \\
\hline siGATA6 \#1 & CCUCUGCACGCUUUCCCUATT & Ambion,Carlsbad,USA \\
\hline siGATA6 \#2 & GGCUCUAUAUGAAACUCCATT & Ambion,Carlsbad,USA \\
\hline shGATA6 \#1 & CCACTACCTTATGGCGTAGAA & Mission (Sigma) \\
\hline shGATA6 \#2 & CTGTCCCTATGACTCCTACTT & Mission (Sigma) \\
\hline
\end{tabular}

\subsection{3 qPCR primers}

\begin{tabular}{|l|l|l|}
\hline GENE & SEQUENCE & SPECIES \\
\hline Ezh2 FP & CAACCCGAAAGGGCAACAAA & Mouse \\
\hline Ezh2 RP & ACCAGTCTGGATAGCCCTCT & Mouse \\
\hline Gata6 FP & CTTTGCGGGCTCTATATGAAACTCCAT & Mouse \\
\hline Gata6 RP & TAGAAGAAGAGGAAGTAGGAGTCATAGGGACA & Mouse \\
\hline Trnp1 FP & TCATCTACGCGGAGGAGTCA & Mouse \\
\hline Trnp1 RP & AGCCTCTGAGGAGCTTAGTGT & Mouse \\
\hline Gata4 FP & CTGTCATCTCACTATGGGCAC & Mouse \\
\hline Gata4 RP & GAGTGACAGGAGATGCATAGC & Mouse \\
\hline Iqgap2 FP & AGCCTCTGAGGAGCTTAGTGT & Mouse \\
\hline Iqgap2 RP & ATGAGGCTTCTGCCATCGAC & Mouse \\
\hline Fam117a FP & CACGGTAACAAAGCCTCCTCT & Mouse \\
\hline Fam117a RP & GGCTCCCGTTTGAAGACGTA & Mouse \\
\hline Pde3b FP & TCACAAGGGATTGAGTGGCAG & Mouse \\
\hline Pde3b RP & AGGCCCATTTAGGTGGCATC & Mouse \\
\hline Inhbb FP & ATCAGCTTTGCAGAGACAGATGG & Mouse \\
\hline Inhbb RP & CTCCGTGACCCTGTTCTTGG & Mouse \\
\hline Sox12 FP & TCGTCTAGTATCGCCGACC & Mouse \\
\hline Sox12 RP & GCCCCAATACCTGATTCCTG & Mouse \\
\hline Creb1 FP & CCTTGGGATATTACAGAAGCTGGAT & Mouse \\
\hline Creb1 RP & CCCTTTAGGCATATTACCTTTGGGA & Mouse \\
\hline Sfrp1 FP & GCAAGCGAGTTTGCACTGAG & Mouse \\
\hline
\end{tabular}


MATERIALS

\begin{tabular}{|c|c|c|}
\hline GENE & SEQUENCE & SPECIES \\
\hline Sfrp1 RP & AGTTGTGGCTGAGGTTGTCC & Mouse \\
\hline KIhl23 FP & CGAGCCCACACCCAGAATATG & Mouse \\
\hline KIhl23 RP & TCATGATGACAGCCCCACAC & Mouse \\
\hline Foxa2 FP & ATCCGCCACTCTCCTT & Mouse \\
\hline Foxa2 RP & CAGTGCCAGTTCTCAC & Mouse \\
\hline Tspan8 FP & GAAGGAAAAGAATCTGCAGGCAC & Mouse \\
\hline Tspan8 RP & AGTCCGTAGAAGGCTGTCCT & Mouse \\
\hline Hdhd3 FP & TCTTTTCCCGAGCCAGGATCT & Mouse \\
\hline Hdhd3 RP & TGTAGGTCAGAGGGGTAGGC & Mouse \\
\hline Ralgps1 FP & GATGGCTAGCGTGTTGGTCA & Mouse \\
\hline Ralgps1 RP & GCCAACTCGGCAAACTCCT & Mouse \\
\hline Txndc16 FP & GAGGGGCATCTTGAAGGCAT & Mouse \\
\hline Txndc16 RP & GCAGACAAGACTGTGATGGGA & Mouse \\
\hline Cxadr FP & AGCCGAGATCGTTTACCTGC & Mouse \\
\hline Cxadr RP & ACTGGTGAAATCCGCGATCC & Mouse \\
\hline Fcgrt FP & ATTAAATGGTCAGAAGAGGGGGAC & Mouse \\
\hline Fcgrt RP & CTCCTCACCATTGAGGGCAAA & Mouse \\
\hline Tgfbr3 FP & CCTCCGCAGTACAGACCAAG & Mouse \\
\hline Tgfbr3 RP & CCTCCGAAACCAGGAAGAGTC & Mouse \\
\hline Tmem51 FP & CTTCGGGATCTCTCGTCTGC & Mouse \\
\hline Tmem51 RP & TCACAGCGGAAAGTCATCCG & Mouse \\
\hline Sort1 FP & CTGACAACAAATGGGTACCGGA & Mouse \\
\hline Sort1 RP & AGCTGGATTCTGGGACAAGC & Mouse \\
\hline RplpO FP & TGGGCAAGAACACCATGATG & $\begin{array}{l}\text { Mouse/ } \\
\text { Human }\end{array}$ \\
\hline RplpO RP & AGTTTCTCCAGAGCTGGGTTGT & $\begin{array}{l}\text { Mouse } \\
\text { /Human }\end{array}$ \\
\hline EZH2 FP & AAAGAACTCACCGAACAGCA & Human \\
\hline EZH2 RP & CAGAAAAGCGTATGAAAGGAGTG & Human \\
\hline GATA6 FP & TCTACAGCAAGATGAATGGCC & Human \\
\hline GATA6 RP & СTCACCСTCAGCATTTCTACG & Human \\
\hline
\end{tabular}




\subsubsection{ChIP Primers}

\begin{tabular}{|l|l|l|}
\hline GENE & SEQUENCE & SPECIES \\
\hline Gata6 TSS/exon FP & AAAAAGCGGCGGTTTCGTTT & Mouse \\
\hline Gata6 TSS/exon RP & GCCTCGGTGAAGAGAGTTCC & Mouse \\
\hline Gata6 intragenic FP & GAGGTCCAAGATCATGTGGCA & Mouse \\
\hline Gata6 intragenic RP & TAGCACTGATTGCCCAAGCC & Mouse \\
\hline Trnp1 TSS/exon FP & AGTCAACAACACCGCACCTT & Mouse \\
\hline Trnp1 TSS/exon RP & TCATCTACGCGGAGGAGTCA & Mouse \\
\hline Trnp1 intragenic FP & AGAGTGGAGCCTCTGAGGAG & Mouse \\
\hline Trnp1 intragenic RP & GTAGAAGTTCTGGGTGGGGC & Mouse \\
\hline Gata4 TSS/exon FP & TCCACCAGCCCAGGAGTTTA & Mouse \\
\hline Gata4 TSS/exon RP & GGAGTGGGAAGAAGTGTCGG & Mouse \\
\hline Gata4 intragenic FP & CCAGTGGAAGGGTCGGTAAC & Mouse \\
\hline Gata4 intragenic RP & TCCCATTCTTGACAAGTGAGGC & Mouse \\
\hline lqgap2 TSS/exon FP & GCTCCTCACCTGATACCCCTA & Mouse \\
\hline lqgap2 TSS/exon RP & TAAGTCCCTCCACCTCGCAA & Mouse \\
\hline lqgap2 intragenic FP & AGCCCTGACTAGGTCAATCCC & Mouse \\
\hline lqgap2 intragenic RP & GGGGCCCTTCAATCCTAACC & Mouse \\
\hline Fam117a TSS/exon FP & AACCGAAAAGTGGCCGAAGT & Mouse \\
\hline Fam117a TSS/exon RP & CAACACTCCTTGCACGCAC & Mouse \\
\hline Fam117a intragenic FP & ATTGCACCTGAGCTGCGT & Mouse \\
\hline Fam117a intragenic RP & CCTTGAGGGCAGAAGGTTCC & Mouse \\
\hline Pde3b TSS/exon FP & CGAGTACCGCGGAGGAAAA & Mouse \\
\hline Pde3b TSS/exon RP & ATAGTAACCGGCTGCGCTTT & Mouse \\
\hline Pde3b intragenic FP & TAGTCCTGTGTTGGCTCCGT & Mouse \\
\hline Pde3b intragenic RP & CACCGTCTTCAGTAACTGCCA & Mouse \\
\hline Inhbb TSS/exon FP & GATGCCAGGCCACTTTTGC & Mouse \\
\hline Inhbb TSS/exon RP & TGCCATTTATCCATCGCCCC & Mouse \\
\hline Inhbb intragenic FP & GAATCGTTTGGCCTTTCCGC & Mouse \\
\hline Inhbb intragenic RP & AACTGACAGGTCACTGGTGC & Mouse \\
\hline Sox12 TSS/exon FP & CGATACTAGACGAGCGCCAG & Mouse \\
\hline Sox12 TSS/exon RP & CCTTCGGGCACGTCACATT & Mouse \\
\hline
\end{tabular}




\begin{tabular}{|l|l|l|}
\hline GENE & SEQUENCE & SPECIES \\
\hline Sox12 intragenic FP & GGCCCTTGTAAGTCAGGTCC & Mouse \\
\hline Sox12 intragenic RP & GGTCCCCATCAAGCAACCAT & Mouse \\
\hline Creb1 TSS/exon FP & GACGCTCCCTCCAGAATGAC & Mouse \\
\hline Creb1 TSS/exon RP & ATGACGCCTCTCGGAACAAC & Mouse \\
\hline Creb1 intragenic FP & AGGTGTGGCTTACTTTGCAGTT & Mouse \\
\hline Creb1 intragenic RP & TGCTCTCTTTCTCTTGTGCCAAA & Mouse \\
\hline Sfrp1 TSS/exon FP & GCGAGTACGACTACGTGAGC & Mouse \\
\hline Sfrp1 TSS/exon RP & CGGGGGCTTGGTGTAGAAG & Mouse \\
\hline Sfrp1 intragenic FP & TGCAGAAACGAGCCAAAAGC & Mouse \\
\hline Sfrp1 intragenic RP & CAGGGCAAGGGTCTGACATT & Mouse \\
\hline KIhl23 TSS/exon FP & TTCTCTCCATCTTGGTGGCATC & Mouse \\
\hline KIhl23 TSS/exon RP & CAATTCGCCGTTGTTGGTTCT & Mouse \\
\hline KIhl23 intragenic FP & AATCCTGTGAGCTAGGGAGGT & Mouse \\
\hline KIhl23 intragenic RP & GTTAGATGGCTGATCTTGAACGATG & Mouse \\
\hline
\end{tabular}

\subsection{Buffers}

\subsubsection{ChIP Buffers}

\subsubsection{Cross linking Buffer}

\begin{tabular}{|l|l|}
\hline COMPONENT & STOCK CONCENTRATION \\
\hline Formaldehyde & $37 \%$ \\
\hline PBS & $1 \mathrm{X}$ \\
\hline
\end{tabular}

\subsubsection{Nelson lysis Buffer}

\begin{tabular}{|l|l|}
\hline COMPONENT & STOCK CONCENTRATION \\
\hline $\mathrm{NaCl}$ & $150 \mathrm{mM}$ \\
\hline EDTA pH 8 & $20 \mathrm{mM}$ \\
\hline Tris pH 7.5 & $50 \mathrm{mM}$ \\
\hline $\mathrm{NP}-40$ & $0.5 \%$ \\
\hline Triton-X-100 & $1 \%$ \\
\hline NaF & $20 \mathrm{mM}$ \\
\hline
\end{tabular}


2.6.1.3 Gomes lysis Buffer

\begin{tabular}{|l|l|}
\hline COMPONENT & STOCK CONCENTRATION \\
\hline $\mathrm{NaCl}$ & $150 \mathrm{mM}$ \\
\hline $\mathrm{NP}-40$ & $1 \%$ \\
\hline Sodium deoxycholate & $0.5 \%$ \\
\hline Tris- $\mathrm{HCl} \mathrm{pH} 8$ & $50 \mathrm{mM}$ \\
\hline EDTA & $20 \mathrm{mM}$ \\
\hline $\mathrm{NaF}$ & $20 \mathrm{mM}$ \\
\hline SDS & $0.1 \%$ \\
\hline
\end{tabular}

2.6.1.4 Gomes Wash buffer

\begin{tabular}{|l|l|}
\hline COMPONENT & STOCK CONCENTRATION \\
\hline Tris-HCl pH 8.5 & $100 \mathrm{mM}$ \\
\hline $\mathrm{LiCl}$ & $500 \mathrm{mM}$ \\
\hline NP-40 & $1 \%(\mathrm{v} / \mathrm{v})$ \\
\hline Sodium deoxycholate & $1 \%(\mathrm{w} / \mathrm{v})$ \\
\hline EDTA & $20 \mathrm{mM}$ \\
\hline NaF & $20 \mathrm{mM}$ \\
\hline
\end{tabular}

2.6.1.5 Weinmann lysis buffer (WB)

\begin{tabular}{|l|l|}
\hline COMPONENT & STOCK CONCENTRATION \\
\hline Tris- $\mathrm{HCl}(\mathrm{pH} 8)$ & $50 \mathrm{mM}$ \\
\hline EDTA & $10 \mathrm{mM}$ \\
\hline SDS & $1 \%$ \\
\hline
\end{tabular}

\subsubsection{Western Buffers}

\subsubsection{Buffers for gels}

\begin{tabular}{|l|l|l|}
\hline SOLUTION & COMPOSITION & AMOUNT \\
\hline \multirow{2}{*}{$\begin{array}{l}6.8 \\
6.8\end{array}$} & Tris-base & $0.5 \mathrm{M}$ \\
\cline { 2 - 3 } Stacking gel buffer & SDS & $0.4 \%(\mathrm{v} / \mathrm{v})$ \\
\hline
\end{tabular}




\section{MATERIALS}

\begin{tabular}{|c|c|c|}
\hline SOLUTION & COMPOSITION & AMOUNT \\
\hline & Acrylamide & $16 \mathrm{ml}$ \\
\hline & Aqua dest & $25 \mathrm{ml}$ \\
\hline \multirow{2}{*}{$\begin{array}{l}\text { Seperating gel buffer stock } \mathrm{pH} \\
8.8\end{array}$} & Tris base & $1.5 \mathrm{M}$ \\
\hline & SDS & $0.4 \%(v / v)$ \\
\hline \multirow{4}{*}{ Separating gel buffer (10 \%) } & Seperating gel buffer stock & $20 \mathrm{ml}$ \\
\hline & Acrylamide & $26.6 \mathrm{ml}$ \\
\hline & Glycerol & $4 \mathrm{ml}$ \\
\hline & Aqua dest & $29.3 \mathrm{ml}$ \\
\hline \multirow{4}{*}{ Separating gel buffer (15 \%) } & Seperating gel buffer stock & $20 \mathrm{ml}$ \\
\hline & Acrylamide & $40 \mathrm{ml}$ \\
\hline & Glycerol & $4 \mathrm{ml}$ \\
\hline & Aqua dest & $16 \mathrm{ml}$ \\
\hline
\end{tabular}

\subsubsection{Transfer buffer}

\begin{tabular}{|l|l|}
\hline COMPONENT & AMOUNT \\
\hline $5 X$ Transfer buffer & $200 \mathrm{ml}$ \\
\hline Ethanol & $200 \mathrm{ml}$ \\
\hline Water & $600 \mathrm{ml}$ \\
\hline
\end{tabular}

\subsubsection{Laemelli Buffer (5X)}

\begin{tabular}{|l|l|}
\hline COMPONENT & STOCK CONCENTRATION \\
\hline Tris- $\mathrm{HCl} \mathrm{pH} \mathrm{6.8}$ & $225 \mathrm{mM}$ \\
\hline Glycerine & $50 \%$ \\
\hline SDS & $5 \%$ \\
\hline DTT & $100 \mathrm{mM}$ \\
\hline Bromophenol blue & $0.02 \%$ \\
\hline B-Mercaptothanol & $5 \%$ \\
\hline
\end{tabular}


2.6.2.4 Running buffer

\begin{tabular}{|l|l|}
\hline COMPONENT & STOCK CONCENTRATION \\
\hline Tris base & $250 \mathrm{mM}$ \\
\hline Glycine & $1.92 \mathrm{M}$ \\
\hline SDS & $1 \%(\mathrm{w} / \mathrm{v})$ \\
\hline
\end{tabular}

\subsubsection{TBS}

\begin{tabular}{|l|l|}
\hline COMPONENT & STOCK CONCENTRATION \\
\hline $\mathrm{NaCl}$ & $150 \mathrm{mM}$ \\
\hline $\mathrm{KCl}$ & $2.68 \mathrm{mM}$ \\
\hline $\mathrm{Na}_{2} \mathrm{HPO}_{4} \times 2 \mathrm{HO}$ & $4.29 \mathrm{mM}$ \\
\hline $\mathrm{KH}_{2} \mathrm{PO}_{4}(\mathrm{pH} 7.4)$ & $1.47 \mathrm{mM}$ \\
\hline
\end{tabular}

\subsubsection{TBST}

\begin{tabular}{|l|l|}
\hline COMPONENT & STOCK CONCENTRATION \\
\hline TBS & $1 \mathrm{X}$ \\
\hline Tween & $0.1 \%(\mathrm{w} / \mathrm{v})$ \\
\hline
\end{tabular}

\subsubsection{WCL Buffer}

\begin{tabular}{|l|l|}
\hline COMPONENT & STOCK CONCENTRATION \\
\hline HEPES pH 7.5 & $50 \mathrm{mM}$ \\
\hline $\mathrm{NaCl}$ & $150 \mathrm{mM}$ \\
\hline EGTA & $1 \mathrm{mM}$ \\
\hline Glycerol & $10 \%(\mathrm{v} / \mathrm{v})$ \\
\hline Triton X-100 & $1 \%(\mathrm{v} / \mathrm{v})$ \\
\hline $\mathrm{NaF}$ & $100 \mathrm{mM}$ \\
\hline $\mathrm{Na} 4 \mathrm{P}_{2} \mathrm{O}_{7 \times 1} \mathrm{H}_{2} \mathrm{O}$ & $10 \mathrm{mM}$ \\
\hline
\end{tabular}




\subsubsection{Blocking solution}

\begin{tabular}{|l|l|}
\hline COMPONENT & STOCK CONCENTRATION \\
\hline TBST & $1 \mathrm{X}$ \\
\hline Skimmed milk & $5 \%(\mathrm{w} / \mathrm{v})$ \\
\hline
\end{tabular}

\subsection{Antibodies, Enzymes and standards}

\subsubsection{Antibodies}

\subsubsection{Antibodies for ChIP and ChIP-Seq}

\begin{tabular}{|l|l|l|}
\hline ANTIBODY & COMPANY & NUMBER \\
\hline EZH2 & diagenode & C15410039-classic \\
\hline H3K27me3 & Cell Signaling & 9733 \\
\hline H3K4me3 & Cell Signaling & 9751 \\
\hline H3K27ac & Genetex & GTX128944 \\
\hline Rabbit IgG & diagenode & C15410206 \\
\hline
\end{tabular}

2.7.1.2 Antibodies for immunohistochemistry

\begin{tabular}{|l|l|l|l|}
\hline ANTIBODY & COMPANY & NUMBER & DILUTION \\
\hline EZH2 (murine) & Cell Signaling & 5246 & $1: 100$ \\
\hline EZH2 (human) & Leica & NCL-L-EZH2 & $1: 300$ \\
\hline HA & Cell Signaling & 3724 & $1: 100$ \\
\hline GATA6 & R\&D Systems & AF1700 & $1: 50$ \\
\hline H3K27me3 & Cell Signaling & 9733 & $1: 200$ \\
\hline
\end{tabular}

\subsubsection{Antibodies for Western Blot}

\begin{tabular}{|l|l|l|l|}
\hline ANTIBODY & COMPANY & NUMBER & DILUTION \\
\hline EZH2 (murine) & Cell Signaling & 5246 & $1: 100$ \\
\hline GATA6 & R\&D Systems & AF1700 & $1: 300$ \\
\hline H3K27me3 & Cell Signaling & 9733 & $1: 1000$ \\
\hline H3 & Abcam & Ab1791 & $1: 1000$ \\
\hline Actin-HRP & Sigma & A3854 & $1: 40000$ \\
\hline
\end{tabular}


MATERIALS

\begin{tabular}{|l|l|l|l|}
\hline ANTIBODY & COMPANY & NUMBER & DILUTION \\
\hline $\begin{array}{l}\text { Anti-rabbit (IgG) } \\
\text { HRP }\end{array}$ & Cell Signalling & 7074 & $1: 10000$ \\
\hline $\begin{array}{l}\text { Anti-mouse (IgG) } \\
\text { HRP }\end{array}$ & Cell Signalling & 7076 & $1: 10000$ \\
\hline $\begin{array}{l}\text { Mouse Anti-goat } \\
\text { (IgG) HRP }\end{array}$ & Santa Cruz & sc-2354 & $1: 5000$ \\
\hline
\end{tabular}

\subsubsection{Enzymes}

\begin{tabular}{|l|l|}
\hline ENZYMES & COMPANY \\
\hline Proteinase K & AppliChem Panreac \\
\hline RNAse A & Sigma-Aldrich \\
\hline
\end{tabular}

\subsubsection{Standards}

\begin{tabular}{|l|l|}
\hline STANDARD & COMPANY \\
\hline DNA ladder $100 \mathrm{bp}$ & Biolabs \\
\hline DNA ladder $1 \mathrm{~kb}$ & ThermoFischer \\
\hline Protein prestained ruler & Thermoscientific \\
\hline
\end{tabular}

\subsection{Cell culture reagents, growth media and antibiotics}

\begin{tabular}{|l|l|}
\hline PRODUCT & COMPANY \\
\hline $\begin{array}{l}\text { Dulbecco's Modified Eagle Medium } \\
\text { (DMEM) }\end{array}$ & $\begin{array}{l}\text { Thermo Fisher Scientific, Waltham, } \\
\text { USA }\end{array}$ \\
\hline Fetal Calf Serum & Biowest, Nuaille, France \\
\hline $\begin{array}{l}\text { Non-Essential Amino Acids Solution } \\
\text { (NEAA-100 X) }\end{array}$ & $\begin{array}{l}\text { Thermo Fisher Scientific, Waltham, } \\
\text { USA }\end{array}$ \\
\hline Trypsin-EDTA (0.5\%) & $\begin{array}{l}\text { Thermo Fisher Scientific, Waltham, } \\
\text { USA }\end{array}$ \\
\hline Puromycin & GIBCO, Invitrogen GmbH, Darmstadt \\
\hline Hygromycin B Gold & InvivoGen, San Diego, USA \\
\hline
\end{tabular}




\section{MATERIALS}

\subsection{Softwares}

\begin{tabular}{|l|l|}
\hline SOFTWARE & COMPANY \\
\hline AUTOsoft 2.6 & Autobio, Zhengzhou, China \\
\hline $\begin{array}{l}\text { Leica Application Suite (LAS) X } \\
\text { Software }\end{array}$ & Leica Camera, Wetzlar, Germany \\
\hline Chemostar Software & $\begin{array}{l}\text { Intas Science Imaging Instruments, } \\
\text { Goettingen, Germany }\end{array}$ \\
\hline StepOne Software & $\begin{array}{l}\text { Thermo Fisher Scientific, Waltham, } \\
\text { USA }\end{array}$ \\
\hline ImageJ & $\begin{array}{l}\text { National Institutes of Health (NIH), } \\
\text { USA }\end{array}$ \\
\hline GraphPad PRISM & GraphPad Software, La Jolla, USA \\
\hline
\end{tabular}




\section{METHODS}

\section{METHODS}

This chapter describes the various methods followed towards performing the experiments and analyses of the acquired data. Studies were conducted with the assistance from and cooperation with different collaborators departments: Tumor grading was performed by Prof. Dr. med Philipp Ströbel, Institute of Pathology, University Medical Center, Goettingen. EZH2 and GATA6 Stainings in Tissue microarray (TMA) samples were provided by Dr. Stefan Kueffer (Institute of Pathology, University Medical Center, Goettingen). The generation of PDAC-PDX models was performed in close collaboration with Prof. Dr. med. Jochen Gaedcke (Department of General-, Visceral- and Pediatric surgery, University Medical Center, Goettingen) and Prof. Dr. med Phillip Ströbel (Institute of Pathology, University Medical Center, Goettingen). The caNFATc1;Kras G12D (NKC) and EZH2 ${ }^{f /+} ; \mathrm{caNFATC1;Kras}{ }^{G 12 D}$ (ENKC) mouse models have been generated with support from Benjamin Steuber, Waltraut Kopp and Sercan Mercan. The shRNA EZH2 NKC cells were obtained from Jinsan Zhang (Gene Regulatory Mechanisms and Molecular Epigenetics Lab, Division of Gastroenterology and Hepatology, Mayo Clinic, Rochester, USA).

\subsection{In vivo experiments}

\subsubsection{Mouse strains}

caNfatc1; Kras ${ }^{G 12 D}$ (NKC) mice have been previously described (Baumgart et al. 2014). Briefly, this model harbors the oncogenic Kras ${ }^{G 12 D}$ mutation and pdx/p48 Cre-mediated expression of HA-tagged NFATc1 constitutively under the control of Rosa26 promoter. Ezh2 $2^{f / f l}$ mice were purchased from Charles River and were interbred with caNfatc1;Kras ${ }^{G 12 D}$ littermates to generate Ezh2 $2^{f / /} ;$ caNfatc1;Kras ${ }^{G 12 D}$ (ENKC) mice. For survival studies,

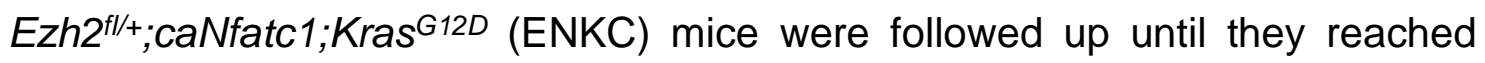
end point criteria and then sacrificed. The pancreas, liver and spleen were collected in $4 \%$ formaldehyde for histological analysis and a part of pancreatic tissue was frozen in $-80{ }^{\circ} \mathrm{C}$. The tissues collected and stored overnight in $4 \%$ formaldehyde were subjected to dehydration in increasing concentrations of ethanol and further embedded in paraffin for long time storage. The tissues in 
paraffin blocks were sectioned with a thickness of $4 \mu \mathrm{m}$ using microtome and fixed on glass slides which were then used for various histological stainings.

All animal procedures were conducted in accordance with the protocols approved by the Institutional Animal Care and Use Committee (33.9-42502-0414/1633; -17/2497 and -19/3085).

\subsubsection{Metastatic incidence in transgenic mice}

For evaluation of micrometastases, three liver sections, each section separated by $20 \mu \mathrm{m}$, from each tumor-bearing caNfatc1;Kras ${ }^{G 12 D_{-}}$and Ezh2 $2^{f /+} ;$ caNfatc1;Kras ${ }^{G 12 D}$ mouse were stained for HA-NFATc1 for better visualization of tumor cells in the liver and were subsequently evaluated for metastasis. Mice that carried at least one HA-positive lesion which was surrounded by healthy liver tissue were considered as positive for metastasis.

\subsection{Cell culture:}

\subsubsection{Cells, culture conditions and Tazemetostat (EPZ6438) treatment}

Primary PDAC cells derived from caNfatc1;Kras ${ }^{G 12 D}$ (NKC) and $K$ ras $^{G 12 D}$;PP53 ${ }^{R 172 H /+}$ (KPC) mice have been described previously (Baumgart et al. 2014; Hingorani et al. 2005). The isolation of pancreatic tumor and further generation of ENKC cell lines from tumor bearing Ezh2 ${ }^{f /+} ;$ caNFATC1;Kras ${ }^{G 12 D}$ mice was performed by Waltraut Kopp. Primary murine PDAC cells were cultured using Dulbecco's Modifies Eagle's Medium (DMEM) containing $4.5 \mathrm{~g} / \mathrm{L}$ D- Glucose, L-Glutamine supplemented with $10 \%$ Fetal calf serum (FCS) and $1 \%$ Non-essential amino acids (NEAA). PANC-1 cells have been previously described (N. M. Chen et al. 2017) and were cultivated using DMEM containing $4.5 \mathrm{~g} / \mathrm{L}$ D-Glucose, L-Glutamine supplemented with $10 \%$ FCS. PDX lines derived from the subcutaneous tumors of PDX mice were cultured in Keratinocyte-SFM (KSF):Roswell Park Memorial Institute (RPMI) (in 3:1 ratio) media supplemented with $2 \%$ FCS, $1 \%$ PenStrep, bovine pituitary extract and epidermal growth factor. Cells were grown in a sterile incubator with $5 \% \mathrm{CO}_{2}$ humidity and at $37^{\circ} \mathrm{C}$. The mycoplasma status of the cells was verified after 


\section{METHODS}

reviving the cells for the experiments using the MycoAlert Mycoplasma Detection Kit from Lonza Group following the instructions mentioned in the kit.

The cells grown up to $80 \%$ confluency were utilized to make cell stocks for long term storage. Cells were trypsinized and centrifuged (1200 rpm for 3 minutes) to pellet down the cells. The supernatant was discarded. Desired amount of cryoprotectant medium (90 \% FCS + $10 \%$ DMSO) was added onto the cells to attain a concentration of $2^{*} 10^{6}$ cells $/ \mathrm{ml}$. Further $1 \mathrm{ml}$ cell suspension was aliquoted into each cryovial and the cryovials were quickly transferred into frosty box (container containing isopropanol). The frosty box was left at $-80{ }^{\circ} \mathrm{C}$ overnight and later transferred into liquid nitrogen for long term storage. While reviving the cells, cryovials were removed from the liquid nitrogen, quickly thawed in a waterbath $\left(37^{\circ} \mathrm{C}\right)$ and resuspended in a falcon containing $10 \mathrm{ml}$ medium. The cell suspension was centrifuged and the supernatant containing freezing medium was discarded. Cells were resuspended in fresh medium and transferred into a cell culture flask. When passaging the cells, cells were briefly washed with PBS to remove the serum. Further, the cells were incubated with trypsin for 3-5 minutes and the reaction was neutralized by adding media on the cells. The content was collected in a falcon, centrifuged (1200 rpm for 3 minutes) and the supernatant was discarded. The cells were then resuspended in fresh medium and split at the required density into a new cell culture flask. EZH2 methyltransferase inhibition was performed by treatment of indicated concentrations of Tazemetostat (EPZ6438) for 72 hours diluted in dimethyl sulfoxide (DMSO). Control cells were treated with equal amounts of DMSO.

\subsubsection{Generation of EZH2 shRNA and CRISPR/Cas9 clones and transient transfection}

CRISPR/Cas9 based genome editing was utilized to generate EZH2 knockout PDAC cells. The PDAC cells were seeded into a $10 \mathrm{~cm}$ dish and once they attained $60 \%$ confluency, they were transfected with pSpCas9(BB)-2A-Puro PX459 vectors containing single guide RNA (sgRNA) against murine or human $E Z H 2$, respectively, with the help of lipofectamine 2000. The following day, cells were split at different dilutions to allow the growth of single colonies and were treated with Puromycin $(2 \mu \mathrm{g} / \mathrm{ml})$ to select for transfected cells. Cells were allowed to grow until they formed visible single colonies on the culture dish. 
Further, the single clones were picked by trypsinizing with small volume of trypsin, taking care not to disturb the neighbouring colonies. The cells were then transferred into 24-well plate and further expanded in culture. Some of these cells were used to harvest for lysate and tested on western blot for knockdown. Further, genomic DNA was isolated from those clones that showed EZH2 knockdown in western blot and a PCR was performed using the genotyping primers. The PCR products were then sequenced by Microsynth Seqlab $\mathrm{GmbH}$ to confirm the knockout. RNA and whole cell lysate (WCL) were also extracted to confirm the knockout at RNA and protein level. After confirmation of successful EZH2 knockout, cells were used for further experiments.

For siRNA or shRNA based knockdowns, siLentFect or Lipofectamine 2000 was used respectively. In short, the designated volume of siRNA and transfection reagent was added to OptiMEM and incubated for 20 minutes and subsequently added on the cells. Media was changed approximately 10 hours post transfection and cells were harvested for experiments after 48-72 hours. For EZH2 re-expression, HA-tagged EZH2 expression plasmid was transfected into NKC cells using lipofectamine 2000 and the transfected cells were selected using the antibiotic Hygromycin $(300 \mu \mathrm{g} / \mathrm{ml})$. The selected cells harboring transient re-expression of wildtype EZH2 were used for experiments.

\subsection{Functional in vitro assays}

\subsubsection{Cell counting assay}

Cell counting assays were performed to determine the trend in cell growth. Experiments were conducted in triplicates. 2,500 cells were seeded in each well of a 6 -well plate (Day 0 ). On day 3 , cells were trypsinized and quantified before replating in a fresh $10 \mathrm{~cm}$ dish. Finally, on day 6 , the cells were trypsinized and counted again.

\subsubsection{BrdU (Bromodeoxyuridine) assay}

BrdU assays were performed to analyze the proliferation capacity of the cells by determining the intensity of absorbance of cellular BrdU incorporation. 2,500 


\section{METHODS}

cells were seeded into each well of a 96-well plate in five replicates per condition. After 72 hours, BrdU labelling reagent was added on the cells and incubated for 4 hours. Further steps were performed according to manufacturer's instructions (BrdU cell proliferation kit, Roche, 11647229001). The absorbance was measured at $405 \mathrm{~nm}$ and the OD values were plotted as BrdU incorporation.

\subsubsection{Boyden chamber assay}

Invasion capacities of PDAC cells were determined utilizing Boyden chamber assay. 50,000 cells were counted and seeded in $50 \%$ matrigel into the collagen coated inserts. The inserts were placed into a 24-well plate. Complete media was added in the lower chamber and serum free media was added into the inserts. After 48 hours, the non-invaded cells and the matrigel were scraped off. The invaded cells were fixed with $4 \%$ paraformaldehyde (PFA) for 20 minutes, washed twice with 1 X PBS, stained with DAPI $(1: 2000)$ and mounted on a glass slide. The DAPI positive nuclei were counted under fluorescence microscope.

When evaluating the invasion potential of GATA6 knockdown cells, the cells transfected with siRNA against GATA6 were trypsinized 24 hours post transfection and seeded for Boyden chamber assay.

\subsubsection{Colony formation assay}

Soft agar assays were performed to evaluate the ability of the cells for anchorage independent growth. The 6 -well plates were coated with 1:1 mix of nobel agar (1\%) and culture medium. Further, 13,000 cells in medium were mixed with $1 \%$ nobel agar in the ratio $1: 3$ and seeded on these coated wells. After ensuring proper solidification of agar (approximately 30 minutes), cell culture medium was added on the top and 7 days later the colonies (cluster of cells visible under microscope) were counted under the microscope.

\subsubsection{Sphere formation assay}

Sphere formation assays were conducted to analyze the stemness behavior of the cells. 30,000 cells were seeded into each well of a 6-well low attachment plate and were supplemented with sphere medium (DMEM/F-12 supplemented 
with $1 \mathrm{X}$ insulin, $0.4 \% \mathrm{BSA}, 20 \mathrm{ng} / \mu \mathrm{l}$ EGF, $10 \mathrm{ng} / \mu \mathrm{FGF}, 2 \%$ B27 supplement and N2 supplement). $0.02 \mathrm{ng} / \mu \mathrm{l}$ fibroblast growth factor (FGF) was added into each well every fourth day. After 11 days, pictures of the spheres from all the wells were taken using phase contrast microscopy. Total number of spheres in each well were counted, and the size of spheres were measured using ImageJ. The average value from three wells were used for plotting the graph.

\subsection{Molecular biology}

\subsubsection{RNA isolation}

The media of cells was aspirated, and the cells were washed twice with $1 \mathrm{X}$ PBS. Further, cells were scraped in TRIzol and collected in a $1.5 \mathrm{ml}$ eppendorf tube. $200 \mu \mathrm{l}$ chloroform was added on the samples, vortexed and centrifuged at $13500 \mathrm{rpm}$ for 15 minutes at $4{ }^{\circ} \mathrm{C}$. The aqueous phase was collected in a separate tube and RNA was precipitated by adding $500 \mu$ isopropanol, allowing it to stand at room temperature (RT) for 15 minutes and centrifuging it at 13500 rpm for 30 minutes at $4{ }^{\circ} \mathrm{C}$. The RNA, which was pelleted down was then washed twice with $75 \%$ ethanol, dried and resuspended in 30-40 $\mu$ laqua-dest water. RNA was stored at $-80{ }^{\circ} \mathrm{C}$. The concentration was measured photometrically using NanoPhotometer P-330 prior to cDNA synthesis or RNA sequencing.

\subsection{2 cDNA synthesis}

$1 \mu \mathrm{g}$ of RNA was reverse transcribed into cDNA using iscript cDNA synthesis kit. cDNA synthesis was performed at $42{ }^{\circ} \mathrm{C}$ for 20 minutes and the enzyme was inactivated by heating the samples at $95{ }^{\circ} \mathrm{C}$ for 1 minute. The sample was further diluted in aqua dest water to $10 \mathrm{ng} / \mu \mathrm{l}$.

\subsubsection{Quantitative polymerase chain reaction (qPCR)}

qPCR was performed to quantify the gene expression. qPCR was performed in triplicates in a reaction volume of $10 \mu \mathrm{l}$. For each reaction, $1 \mu \mathrm{l}$ of cDNA or DNA from ChIP was mixed with $3.9 \mu \mathrm{H}_{2} \mathrm{O}, 5 \mu \mathrm{l}$ of SYBR green mix and $0.05 \mu \mathrm{l}$ of each forward and reverse primers, and added into each well of a MicroAmp 


\section{METHODS}

Fast Optical 96-well reaction plate. The plate was sealed with an adhesive film and was briefly vortexed before placing it into StepOnePlus Real-Time PCR System. The PCR reaction was as follows:

\begin{tabular}{|l|l|l|}
\hline $95^{\circ} \mathrm{C}$ & 10 minutes & \\
\hline $95^{\circ} \mathrm{C}$ & 15 second & \multirow{2}{*}{40 cycles } \\
\cline { 2 - 2 } $60^{\circ} \mathrm{C}$ & 1 minute & \\
\hline
\end{tabular}

The CT values obtained were used to calculate the expression. The expression was normalized to the housekeeping gene Rp/pO and further normalized to control using the $\triangle \triangle C T$ method. For ChIP, qPCR samples were normalized corresponding to their ChIP input samples.

\subsubsection{Chromatin Immunoprecipitation (ChIP)}

\subsubsection{Cross linking and sonication}

Cells were grown in a $15 \mathrm{~cm}$ dish and were used for ChIP once they attained $80 \%$ confluency. Cells were fixed with $1 \%$ formaldehyde in PBS for 20 minutes and the reaction was quenched by adding $1.25 \mathrm{M}$ glycine for 5 minutes. Further, cells were washed twice with ice-cold PBS before adding $1 \mathrm{ml}$ Nelson buffer containing protease inhibitors on the cells. The cells were scraped, collected in a $1.5 \mathrm{ml}$ eppendorf tube and centrifuged at $11000 \mathrm{rpm}, 4$ ${ }^{\circ} \mathrm{C}$ for 5 minutes. The nuclei which had formed a pellet were washed once more with $1 \mathrm{ml}$ Nelson buffer. The final pellet was resuspended in $200 \mu \mathrm{l}$ Gomes lysis buffer containing protease inhibitors and SDS, and then incubated on a rotating wheel at $4{ }^{\circ} \mathrm{C}$ for 15 minutes. The samples were sonicated using Biorupter with 30 seconds ON/OFF pulse for 25 cycles. The sonicated samples were then centrifuged at $13000 \mathrm{rpm}$ for 10 minutes at $4{ }^{\circ} \mathrm{C}$ and the supernatant was used to proceed with pre-clearing.

\subsubsection{Shearing check}

Shearing check was performed to confirm that the sonication has sheared the chromatin efficiently before proceeding with pre-clearing step. $10 \mu \mathrm{l}$ of sonicated samples was incubated overnight on a shaker $\left(65^{\circ} \mathrm{C}, 800 \mathrm{rpm}\right)$ in $100 \mu \mathrm{l}$ Weinmann lysis buffer supplemented with $30 \mu \mathrm{g}$ proteinase $\mathrm{K}$. On the 
following day, $100 \mu \mathrm{l}$ water, $10 \mu \mathrm{LiCl}(8 \mathrm{M})$ and $2 \mu \mathrm{l}$ glycogen was added into the sample and DNA was isolated by adding phenol/chloroform/isoaymyl alcohol $(200 \mu \mathrm{l})$ and centrifuging at full speed for 2 minutes. The aqueous phase was collected and the phenolic phase underwent back extraction with $200 \mu \mathrm{l}$ Tris $(\mathrm{pH} \mathrm{8)} 10 \mathrm{mM}+0.4 \mathrm{M} \mathrm{LiCl}$ followed by 2 minutes full speed centrifugation. The aqueous phase was added into the first tube and the DNA was precipitated using $100 \%$ ethanol. The DNA pellet was washed once with $70 \%$ ethanol then finally resuspended in aqua-dest water with RNAse A (100 $\mu \mathrm{g} / \mathrm{ml})$. Further, the DNA was mixed with loading dye and run on a $1.5 \%$ agarose gel. The gel was observed under the gel documentation system and the shearing was considered efficient if most of the fragments were in the range 200-500 bp.

\subsubsection{Pre-clearing and Immunoprecipitation}

Required volume of Agarose A beads was washed twice with Gomes lysis buffer. The ChIP samples were brought up to $500 \mu \mathrm{l}$ in Gomes lysis buffer and were incubated with $100 \mu$ Agarose A beads $50 \%$ slurry for 1 hour at $4{ }^{\circ} \mathrm{C}$. Afterwards, the sample were centrifuged and the supernatant was aliquoted for IP and one tube ( $2 \%$ of the total sample) was saved as input.

The chromatin aliquots were incubated with desired antibodies mix $(1-2 \mu \mathrm{g} / \mathrm{IP})$ at $4{ }^{\circ} \mathrm{C}$ overnight on a cogwheel. On the following day, the blocked beads (Agarose A beads were blocked in Gomes lysis buffer with $1 \mathrm{mg} / \mathrm{ml} \mathrm{BSA}$ overnight) were added and incubated for an hour. Further, the ChIP immune complex were washed once with Gomes lysis buffer, twice with Gomes wash buffer, twice with Gomes lysis buffer and finally with 1X TE buffer.

\subsubsection{DNA isolation}

The ChIP samples as well as input samples were diluted with $10 \mathrm{mM}$ Tris $(\mathrm{pH}$ 8) containing $10 \mu \mathrm{g}$ RNAse $\mathrm{A}$ and incubated for 30 minutes at $37^{\circ} \mathrm{C}$. Further, $50 \mu \mathrm{l}$ Weinmann lysis buffer with an additional $30 \mu \mathrm{g}$ proteinase $\mathrm{K}$ were added and incubated at $65{ }^{\circ} \mathrm{C}$ overnight. The samples were centrifuged and the supernatant was collected in a separate tube. The DNA on the beads was eluted by resuspending with $100 \mu \mathrm{l}$ Tris $(\mathrm{pH} 8)$, incubating for 10 minutes at 65 ${ }^{\circ} \mathrm{C}, 800 \mathrm{rpm}$ followed by centrifuging and collecting the supernatant into the first 


\section{METHODS}

tube. The DNA was further extracted using the phenol/chloroform/isoaymyl alcohol extraction method (same as mentioned in shearing check). Finally, the DNA was resuspended in $40 \mu$ aqua-dest water. qPCR was performed with the input and ChIP samples and further the expression was normalized to expression in input samples.

\subsection{Protein biochemistry}

\subsubsection{Sample preparation and Bradford Assay}

For protein analyses, cells were harvested in whole cell lysis buffer supplemented with $1 \mathrm{X}$ cOmplete ${ }^{\mathrm{TM}}$ protease inhibitor cocktail and additional protease inhibitors- PMSF, NaF and $\mathrm{NaO}$. Cells were scraped, collected in an eppendorf tube, incubated for 30 minutes on ice and centrifuged at $13000 \mathrm{rpm}$ for 30 minutes at $4{ }^{\circ} \mathrm{C}$. The supernatant was collected in a fresh eppendorf tube which was stored at $-20{ }^{\circ} \mathrm{C}$ for short time period and at $-80{ }^{\circ} \mathrm{C}$ for longterm storage. Protein concentration was measured using Bradford reagent following standard protocol. Different dilutions of Bovine serum albumin (BSA) was used as the standard. The absorbance of the standards and samples were measured at $595 \mathrm{~nm}$. The absorbance value of the standards were plotted and the coefficent was derived which was used to calculate the concentration of the sample.

\subsubsection{SDS PAGE and Western blot analysis}

Sodium dodecyl sulfate polyacrylamide gel electrophoresis (SDS-PAGE) was performed to separate the proteins based on their molecular weight. We used a $10 \%$ gel to detect proteins of size larger than $30 \mathrm{kDa}$ and a $15 \%$ gel to visualize proteins smaller than $30 \mathrm{kDa}$. To generate the polyacrylamide gels, the plates were assembled together in the cassette. Approximately $4.5 \mathrm{ml}$ resolving gel solution was poured in the spacer within two plates and was overlayed with stacking gel solution. 10 well comb was placed and the gel was allowed to solidify for 30-45 minutes. Further the comb was carefully removed, gel was placed in the electrophoresis tank and the tank was filled with running buffer. Equal amount of proteins were incubated with SDS and heated up at 95 
${ }^{\circ} \mathrm{C}$ for 5 minutes. These samples were loaded into the wells of the gel and the electrophoresis was performed at 120 volts for approximately 1 hour. Once the proteins were separated by polyacrylamide gel electrophoresis (PAGE), the proteins on the gel were transferred onto a nitrocellulose membrane using a TurboBlot system (fixed: $25 \mathrm{~V}$ and $1.0 \mathrm{~A}$, time: 22 minutes for $10 \%$ gels and 10 minutes for $15 \%$ gels). Further, the membrane was briefly stained with Ponceau $S$ solution to visualize the proteins bands thereby to confirm the blotting. The membrane was blocked using $5 \%$ milk in TBST and incubated with primary antibodies overnight at $4{ }^{\circ} \mathrm{C}$. Membranes were then incubated with respective secondary antibodies for 1 hour at room temperature. Bands were visualized in Intas ECL Chemocam Imager using chemiluminescence.

\subsection{Next generation sequencing:}

\subsubsection{ChIP seq library preparation and analysis}

For ChIP-seq, ChIP was performed as described above and with antibodies against EZH2, H3K27me3 and H3K4me3. IgG was used as a control. Subsequently, DNA samples were sonicated in a Biorupter® Pico to obtain approximately $300 \mathrm{bp}$ fragments which were used for library preparation with the MicroPlex Library Preparation Kit as per manufacturer's protocol. Sequencing was performed using the HiSeq 2000 Illumina platform of the NIG. Subsequently, the ChIP-seq files were analyzed in the public server (usegalaxy.org). The quality of the raw files were assessed by running FASTQ quality check (FASTQC). After ensuring the quality of the raw data, the sequence reads were aligned to the mouse reference genome (mm9) using Bowtie2 (version 0.4) with default parameters (Langmead and Salzberg 2012) and the aligned reads were saved as a BAM file. This file was used for peak calling utilizing the Model based Analysis of ChIP-seq (MACS2 version 2.1.0.20151222) tool (Zhang et al. 2008) on UseGalaxy (usegalaxy.org) server. The cut off value for peak detection was set to 0.05 . BigWig files were generated using BamCoverage (version 2.2.3) from deeptools (Ramírez et al. 2016) and were visualized using integrative genomics viewer (IGV version 2.5.3) (Robinson et al. 2011). Further, BigWig and bed files were used to generate aggregate profiles and distribution of ChIP regions on chromosome 46 


\section{METHODS}

using CEAS tool (Shin et al. 2009) on Galaxy cistrome. Differential binding analysis was performed to identify differentially occupied regions in shRNA EZH2- compared to shRNA control NKC cells using the Bioconductor $R$ package Diffbind (Ross-Innes et al. 2012) run on $R$ version 3.6.1 according to the instruction manual. Furthermore, Genomic Regions Enrichment of Annotations Tool (GREAT) analysis (Mclean et al. 2010) was used to identify associated genes with regions identified by Diffbind analysis. The default association rule, basal plus extension (5 kb upstream, $1 \mathrm{~kb}$ downstream plus distal $1 \mathrm{Mb}$ ), was set for GREAT analysis. Further, principle component analysis (PCA) for the H3K27me3 and H3K4me3 profiles was plotted in R. Heatmaps and average profiles for occupancy were generated using the computeMatrix and plotHeatmap tools on the UseGalaxy server and peak center was set as the reference point mode.

For analyses in PANC-1 cells, input and EZH2 ChIP-seq sequence reads were aligned against the human reference genome (hg38) and BigWig files were generated as described above. The input peaks were subtracted from the EZH2 binding peaks and visualized in IGV.

\subsubsection{RNA seq library preparation and analysis}

ShRNA control and shRNA EZH2 NKC cells were cultured in triplicates and later harvested in TRIzol. RNA was isolated by phenol-chloroform method followed by confirming the purity and integrity on $1 \%$ agarose gel. $500 \mathrm{ng}$ of total RNA was used to prepare libraries using True seq RNA library preparation kit as per the manufacturer's instructions. cDNA library concentrations and fragment sizes were controlled by Qubit and bioanalyzer respectively prior to sequencing in the NGS Integrative Genomics Core Unit (NIG) of the UMG. The FastQ files from the RNA sequencing were analyzed in the public server usegalaxy.org. The reads were aligned to the murine transcriptome mm9 using TopHat2 (version 2.1.0) (D. Kim et al. 2013). Further, differential gene regulation and Fragment Per Kilobase Million (FPKM) values were obtained by Cuffdiff (version 2.2.1) and Cuffnorm (version 2.2.1.1) respectively (Trapnell et al. 2013). The read counts obtained by HTSeq (version 0.9.1) (Anders, Pyl, and Huber 2015) were used to plot Principal Component Analysis (PCA) in R. Genes with FPKM of $<0.2$ were excluded from the analysis to reduce 
background signals. Gene ontology was performed using PANTHER GO Ontology database (Binomial test with Bonferroni correction) and pathways with FDR $<0.05$ were considered as significant pathways. GSEA was performed using standard parameters (Signal2Noise metric for gene ranking).

\subsection{H\&E staining and immunohistochemistry}

Upon sacrificing the mice, pancreas and liver tissue were collected in parafolmaldehyde, dehydrated in increasing concentrations of ethanol and further embedded in paraffin to make tissue blocks. Paraffin blocks were sectioned into $4 \mu \mathrm{m}$ sections and fixed on glass slides. Hematoxylin \& Eosin (H\&E) stainings were performed to visualize the tissue morphology. To this end the slides were placed in roticlear to remove the paraffin (de-waxing). Further, the tissue sections were hydrated by placing the slides in decreasing concentrations of ethanol (99\%, $96 \%, 80 \%, 70 \%, 50 \%)$. The slides were placed in hematoxylin solution, which stains the nuclei blue, for approximately 5 minutes and then rinsed with tap water for 7 minutes. Further, the slides were transferred to the eosin in $0.2 \%$ acetic acid solution, which consists of a dye that stains the cytoplasm, for approximately 10 seconds. The sections were further dehydrated by placing in increasing concentrations of ethanol $(70 \%, 80$ $\%, 96 \%, 99 \%$ ) and finally in roticlear. The slides were mounted using a cover slip and with a mounting solution and further viewed under the microscope.

For immunohistochemistry $(\mathrm{IHC})$, the sections were de-waxed by placing the slides in roticlear and further hydrated by placing in decreasing concentrations of ethanol. Then, the slides were covered with TE/citrate buffer and boiled for 510 minutes in the microwave to expose the antigens and then cooled down by placing it on the ice. Further the sections were incubated in $3 \%$ hydrogen peroxide solution for 10 minutes to block the endogenous peroxidase activity. The slides were placed in the cadenxa slide holding system and then blocked with $10 \%$ BSA solution prepares in PBST (containing $1 \%$ Tween or $1 \%$ TritonX). The slides were then incubated overnight with the primary antibody. The following day, slides were washed twice with PBST and incubated with the secondary antibody for 1 hour, followed by incubating with $A B$ complex for another hour (performed utilizing the Peroxidase Rabbit/Mouse IgG Vectastain 48 


\section{METHODS}

ABC kits). The slides were then washed with PBST and stained with DAB solution. The reaction was stopped by placing the slides in water and further the slides were counterstained in hematoxylin for 5 minutes. Slides were placed under tap water for 7 minutes for the nuclei stain to develop into blue colour. Finally, sections were dehydrated and mounted as explained for H\&E stainings. The slides were viewed under the microscope.

\subsection{Tissue Microarray Analysis (TMA) in human PDAC samples}

EZH2 and GATA6 stainings in TMA were provided by the Institute of Pathology, UMG, Göttingen. TMAs were prepared from 54 resected PDAC patients with three to four cores per patient. TMAs were evaluated for nuclear EZH2 and GATA6 expression and were scored in accordance to the Immune Reactive Sore (IRS). Each tissue was given a score for the percentage of positively stained cells $(A)$ and intensity of staining (B). Multiplying the scores of two variables $\left(A^{*} B\right)$ gave a IRS ranging from 0 to 12 . Tissues with IRS $\leq 3$ were considered as tissues with null or mild staining and the tissues with IRS $>3$ were considered as moderate or strongly stained.

\subsection{Statistical analysis}

Statistical analysis was performed by Graphpad Prism (version 6.0). Data are represented as mean \pm standard deviation (SD). Fisher's exact test was used to analyze the significance of in vivo data. Log-Rank test was used for the survival analysis. Two-tailed unpaired student's $t$-test, one-way and two-way ANOVA were used calculate the statistical significance of in vitro data (described in each figure legend). $p$ value of $<0.05$ was considered as statistically significant. $p$ - values $<0.05,<0.01$ and $<0.001$ are depicted as *, ${ }^{* *}$ and ${ }^{* * *}$, respectively. 



\section{RESULTS}

\subsection{EZH2 in tumor progression}

\subsubsection{EZH2 is overexpressed in dedifferentiated human PDAC}

In order to elucidate the role of EZH2 in PDAC, we initially examined EZH2 expression in human PDAC samples and adjacent normal pancreatic tissue. To this end we conducted EZH2 IHC in a Tissue Microarray (TMA) of resected PDAC samples from 54 patients. Consistent with previous reports (Ougolkov, Bilim, and Billadeau 2008), EZH2 was almost absent in the normal pancreas whereas its expression increased in the epithelial part of PDAC tissues (Figure $5 A)$.

Next, we used the immunoreactive score (IRS) to correlate EZH2 expression with the tumor grading information of the respective donor patient as provided by the Institute of Pathology. The IRS scoring system considers the percentage of positively stained cells and the intensity of the staining (Fedchenko and Reifenrath 2014). A significant number of patients with low EZH2 levels possessed low grade pancreatic tumors. Overall, there was a significant association between high EZH2 levels with high-grade tumors (Figure 5B). Together, these analyses suggest overexpression of EZH2 in PDAC vs normal pancreatic tissue and implicate an association of EZH2 expression with PDAC dedifferentiation. 


\section{RESULTS}

(A)

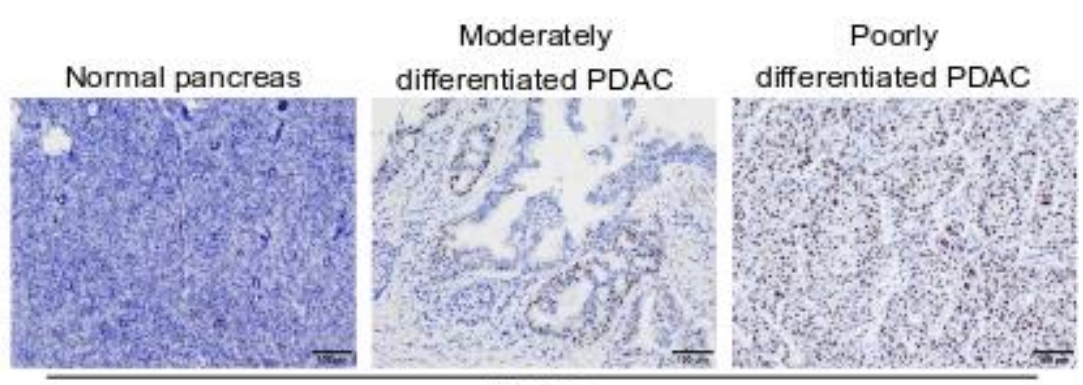

EZH2

(B)

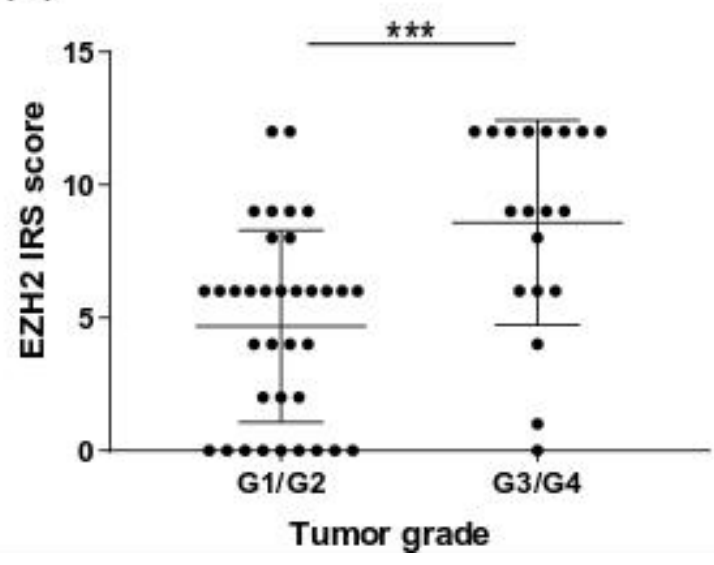

Figure 5: EZH2 expression correlates with high grade PDAC.

(A) Representative immunohistochemical analysis of EZH2 expression in healthy pancreatic tissue, moderately and poorly differentiated PDAC from a TMA performed in human PDAC patients ( $\mathrm{n}=54$ patients). Magnification 100X, Scale bar: $100 \mu \mathrm{m}$. (B) Correlation of EZH2 expression (IRS score) and tumor grading in the same TMA. Each dot represents a patient. Values represent mean \pm SD. Significance was determined by two-tailed unpaired student's $t$-test.

\subsubsection{EZH2 drives PDAC progression in transgenic mice}

Given the observations in human PDAC tissues, we sought to dissect the functional implications of EZH2 in vivo using transgenic mouse models. We utilized the caNfatc1;Kras ${ }^{G 12 D}$ (NKC) mice which have been previously described (Baumgart et al. 2014). Briefly, this transgenic mouse model has the gatekeeper Kras ${ }^{G 12 D}$ mutation and constitutive expression of HA-tagged NFATc1 in the pancreas. NFATc1 is a well-studied inflammatory transcription factor and in combination with Kras ${ }^{G 12 D}$ gives rise to PDAC with a penetrance of nearly $100 \%$. Importantly, PDAC that develops in the NKC model very well 


\section{RESULTS}

mimics human PDAC with frequent occurrence of liver metastasis, ascites and bile obstruction (Baumgart et al. 2014).

To investigate the impact of EZH2 expression in this mouse model, caNfatc1;Kras ${ }^{G 12 D}$ mice were crossed with Ezh2t/fl mice to obtain Ezh2 $2^{f / f f ;}$;caNfatc1;Kras ${ }^{G 12 D}$ littermates. Surprisingly, homozygous Ezh2 $2^{f / f f} ;$ caNfatc1;Kras ${ }^{G 12 D}$ animals died 5-10 days postnatally. Necropsy of mice showed signs of liver inflammation, severe pancreatic atrophy or complete loss of the pancreatic organ (Figure 6), indicating that in the context of constitutively active NFATc1, EZH2 is vital for the development of a morphologically and functionally intact pancreas.

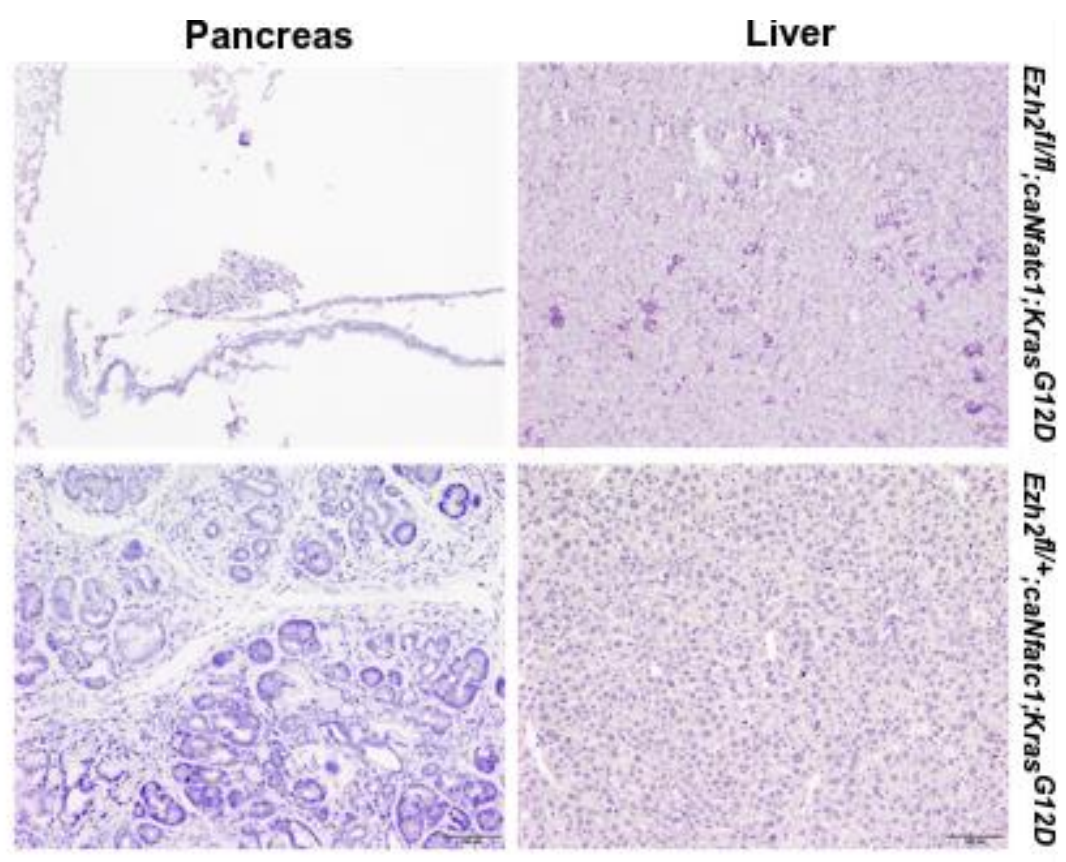

Figure 6: Complete loss of EZH2 hampers pancreatic development in mice.

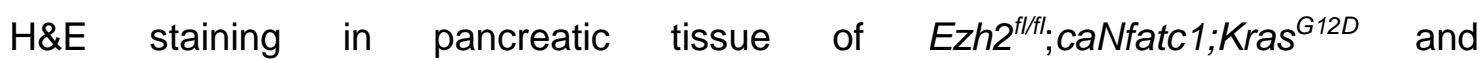
Ezh2 $2^{f /+} ;$ caNfatc1; Kras ${ }^{G 12 D}$ mice displaying severe pancreatic atrophy and liver inflammation in animals with homozygous Ezh2 depletion. Magnification 100X, Scale bar $100 \mu \mathrm{m}$. 


\section{RESULTS}

As the homozygous Ezh2 knockout mice could not be used for the study, we interbred Ezh2 ${ }^{f / f t} ;$ caNfatc1;Kras ${ }^{G 12 D}$ mice with Ezh2 ${ }^{+l_{+}}$(wildtype) animals to obtain Ezh2f//;caNfatc1;Kras ${ }^{G 12 D}$ mice with heterozygous EZH2 expression. Both caNfatc1;Kras ${ }^{G 12 D}$ and Ezh2f//+;caNfatc1;Kras ${ }^{G 12 D}$ (ENKC) mice gave rise to all steps of pancreatic carcinogenesis. ADM and PanIN lesions were observed in caNfatc1; Kras ${ }^{G 12 D}$ mice as soon as 2 weeks after birth. While most of the 8 week old mice displayed the full PanIN PDAC precursor lesions, nearly all 7 month old animals suffered from PDAC. The pancreas of 8 week old Ezh2fl/+;CaNfatc1;Kras ${ }^{G 12 D}$ animals displayed ADMs and PanIN precursor lesions and few of these mice developed PDAC.

The ADM, PanIN lesions and PDAC of caNfatc1;Kras ${ }^{G 12 D}$ and Ezh2fl/+;caNfatc1;KrasG12D mice were subjected to various immunohistochemical analyses. As this mouse model has overexpression of HA-tagged NFATc1, tissues were stained for HA-tag thereby validating the expression of NFATc1 and to distinguish epithelial- from non-epithelial tissue parts. Consistent with the observations in human pancreatic cancer tissues, EZH2 was nearly absent in acinar cells but showed increased expression and activity (as illustrated by H3K27me3 staining) in PDAC precursor lesions and established tumors. Interestingly, PDAC developing in Ezh2 ${ }^{f / /+} ;$ caNfatc1;Kras ${ }^{G 12 D}$ mice were overall more differentiated than the tumors in caNfatc1; Kras ${ }^{G 12 D}$ mice (Figure 7), suggesting that EZH2 promotes dedifferentiation in these mice. 


\section{RESULTS}

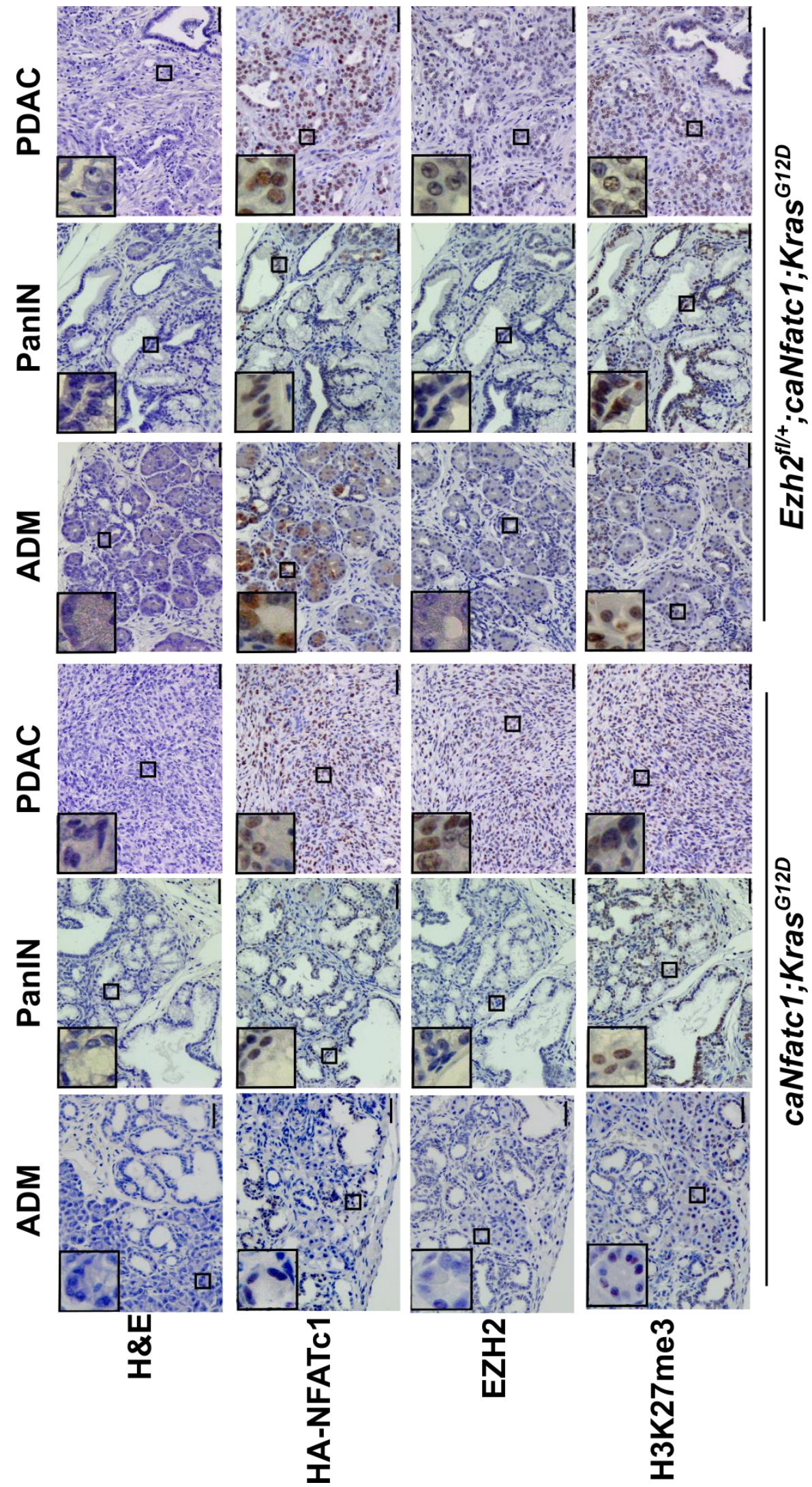

Figure 7: EZH2 drives PDAC development and dedifferentiation.

Representative images of $\mathrm{H} \& \mathrm{E}$ and other immunohistochemical stainings (HANFATc1, EZH2, H3K27me3) in ADM, PanIN lesions and PDAC of caNfatc1;Kras ${ }^{\mathrm{G} 12 D}$ (NKC) and Ezh2 ${ }^{t /+;}$ caNfatc1;Kras ${ }^{G 12 D}$ (ENKC) mice. Magnification 200X, Scale bar 50 $\mu \mathrm{m}$. 


\section{RESULTS}

Mice were followed till the end point criteria were reached and then sacrificed. The end point was defined as mice experiencing weight loss of more than $20 \%$ or showing signs of pain. The pancreas tissue of the mice was stained with $H \& E$ and was evaluated by a pathologist to determine which tissue contains PDAC. Remarkably, caNfatc1;Kras ${ }^{G 12 D}$ mice developed PDAC with almost 100

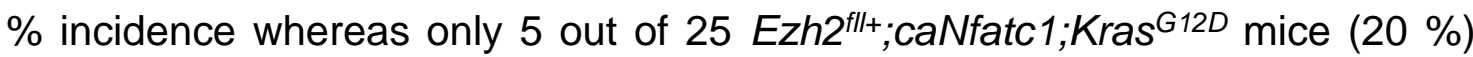
developed PDAC (Figure 8A). Furthermore, Kaplan Meier plot was plotted to compare the survival between the two groups of mice. Surprisingly, and despite the EZH2-status dependent differences in PDAC development,

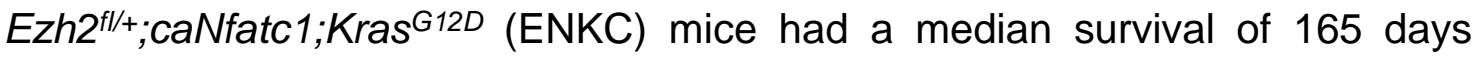
which was comparable to that of caNfatc1;Kras ${ }^{G 12 D}$ mice, which displayed a median survival of 145 days (Figure 8B). Ezh2fl/; $;$ caNfatc1;Kras ${ }^{G 12 D}$ mice displayed initial signs of pancreatic insufficiency and atrophy at an advanced age, potentially explaining the insignificant differences seen in the survival of these mice.
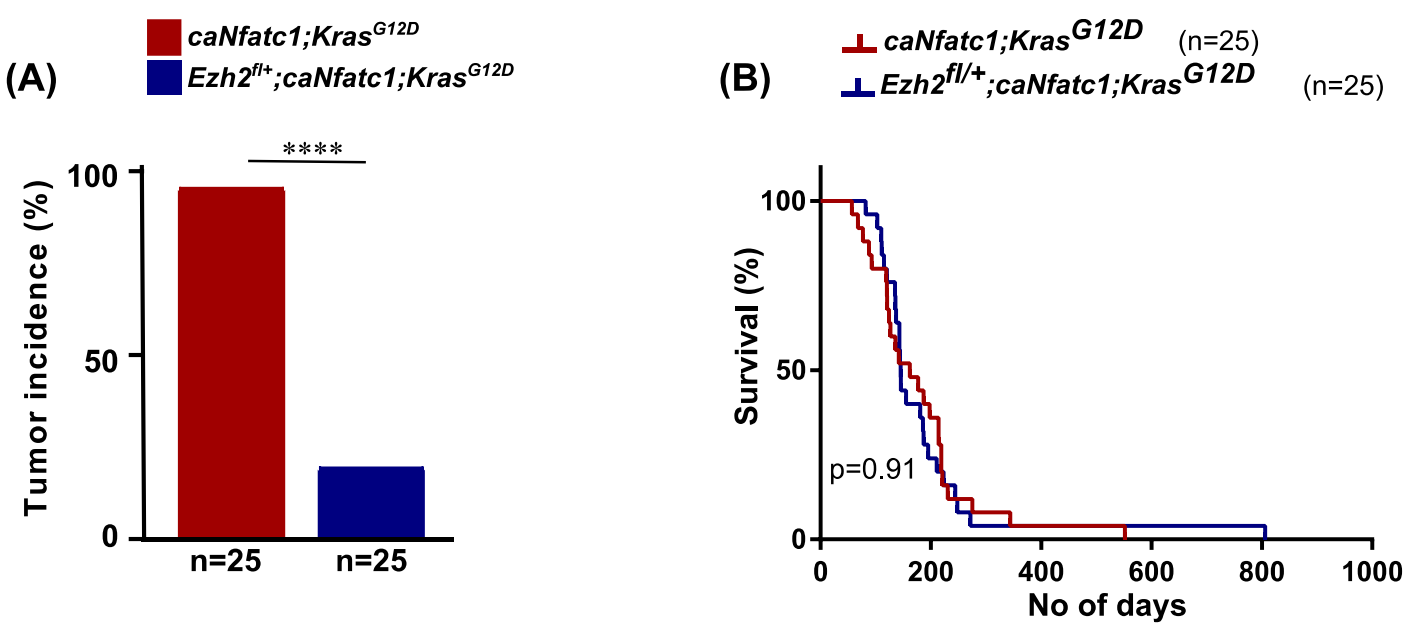

Figure 8: EZH2 correlates with increased PDAC incidence in transgenic mice

(A) Bar graph showing PDAC incidence in survival mice of indicated genotypes. 25 mice per genotype were enrolled into the study. Significance was determined by twotailed Fisher's exact test (B) Kaplan-Meier plot displaying survival of Ezh2 $^{f / /+} ;$ caNfatc1;Kras ${ }^{G 12 D}$ (ENKC) mice (median survival 162 days) compared to that of caNfatc1; Kras ${ }^{G 12 D}$ (NKC) mice (median survival 145 days). Significance was determined by Log-rank (Mantel-Cox) test. 


\section{RESULTS}

\subsubsection{EZH2 drives liver metastasis in transgenic mice}

Tumor dedifferentiation is associated with invasion and metastasis. The liver is the most common site for distant metastasis in pancreatic cancer patients (Yachida and Lacobuzio-Donahue 2009). Therefore, tumor-bearing mice from both genotypes were evaluated for liver metastasis. Three liver sections from each tumor-bearing mouse from both groups were stained for HA-NFATc1 for better visualization of PDAC cells in the liver. Mice that carried at least one HApositive lesion in the liver which was surrounded by healthy liver tissue were considered positive for metastasis. While $33 \%$ of tumor-bearing caNfatc1;Kras ${ }^{G 12 D}$ mice displayed liver metastasis, only $20 \%$ of Ezh2 $2^{f /+} ;$ caNfatc1;Kras ${ }^{G 12 D}$ mice developed metastasis (Figure 9). This suggests a possible role of EZH2 in promoting metastasis.

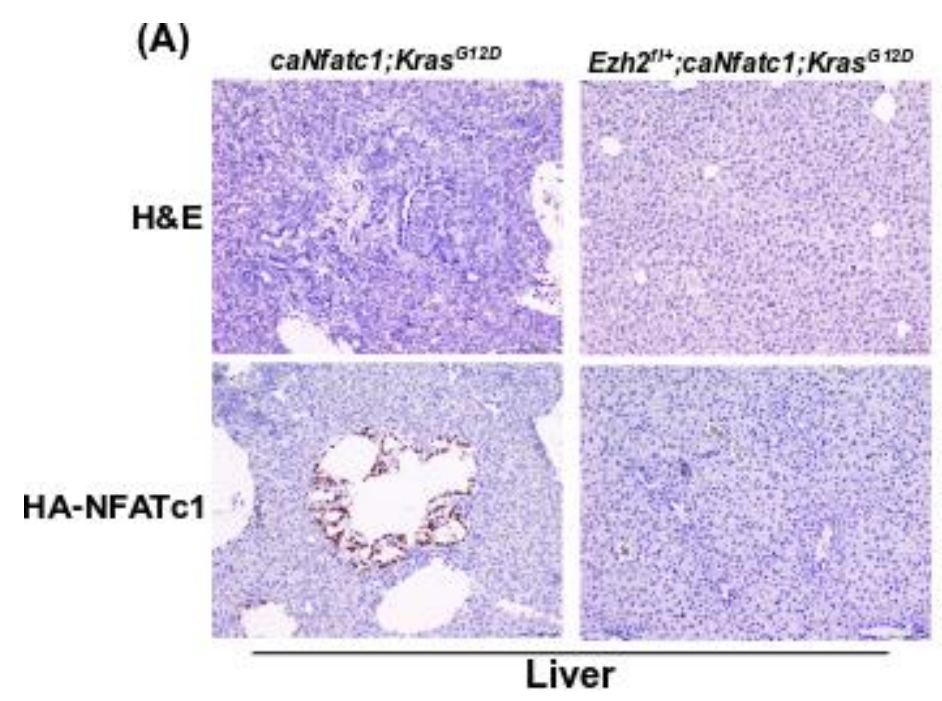

(B)

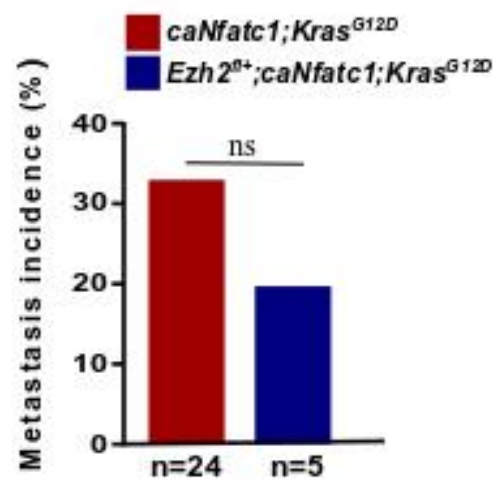

Figure 9: EZH2 deficiency reduces metastatic incidence.

(A) Representative images of H\&E and HA-NFATc1 staining in liver sections of caNfatc1; Kras ${ }^{G 12 D}$ (NKC) and Ezh2 ${ }^{\text {f// }} ;$ caNfatc1;Kras ${ }^{G 12 D}$ (ENKC) mice. Magnification 100X, Scale bar $100 \mu \mathrm{m}$. (B) Bar graph showing percentage of tumor bearing mice from indicated genotypes carrying liver metastasis. Significance was determined by two-tailed Fisher's exact test. 
Together, these results in human PDAC samples and transgenic mouse models suggest a critical involvement of $\mathrm{EZH} 2$ in driving tumor progression and dedifferentiation towards a highly aggressive PDAC phenotype.

\subsection{Functional implications of EZH2 in PDAC cells}

To delineate the functional implications of EZH2 in PDAC maintenance, we isolated pancreatic tumors from the EZH2-expressing caNfatc1;Kras ${ }^{G 12 D}$ mice and further harvested primary PDAC cells (NKC cells) from the tumor (Baumgart et al. 2014). NKC cells were subjected to shRNA mediated knockdown of EZH2 to generate stable EZH2 knockdown NKC cells (Figure 10A). The western blot confirms the reduction in EZH2 expression and subsequent decrease in H3K27me3 levels upon EZH2 knockdown (Figure 10B). The shRNA control and shRNA EZH2 NKC cells were utilized to perform various functional assays. 


\section{RESULTS}

(A)

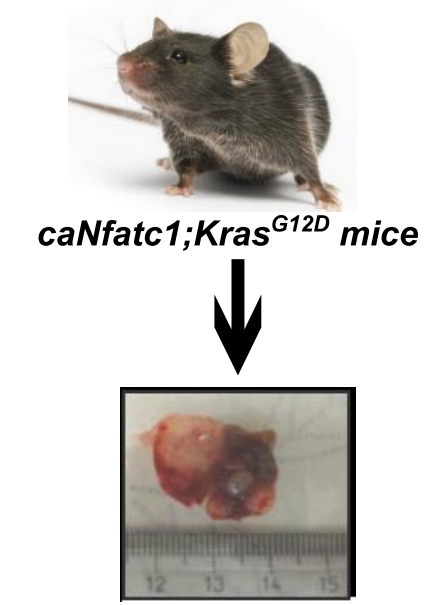

PDAC tumor

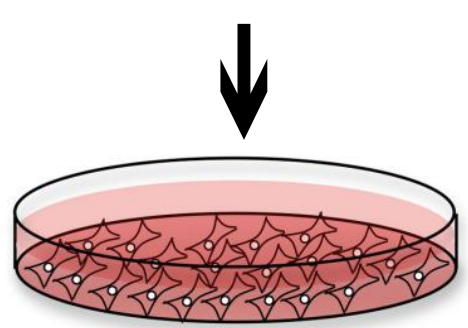

Primary PDAC cells

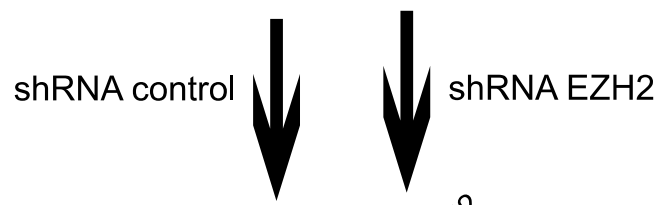

(B)

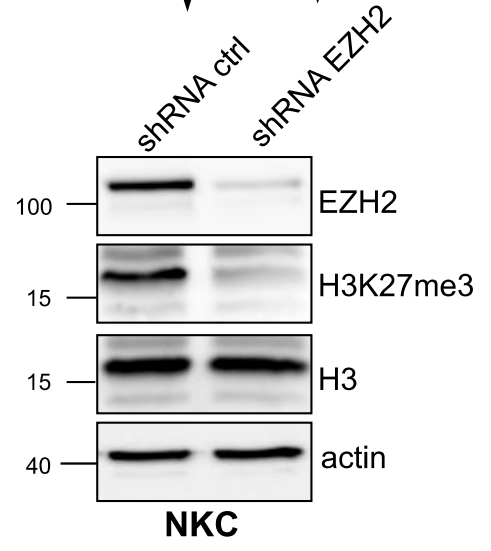

Figure 10: Generation of shRNA-mediated stable knockdown of EZH2 in NKC cells.

(A) Schematic displaying the isolation of primary PDAC cells (NKC cells) from caNfatc1;Kras ${ }^{G 12 D}$ (NKC) mice and further generation of shRNA-mediated stable knockdown of EZH2 in these cells. (B) Western blot depicting reduced EZH2 and H3K27me3 expression in NKC cells upon stable shRNA-mediated EZH2 depletion. H3 and actin serve as loading controls. 


\subsubsection{EZH2 depletion reduces proliferation in NKC cells}

Uncontrolled cell proliferation is an important property of cancer cells (Hanahan and Weinberg 2011) and previous studies have investigated the involvement of EZH2 in mediating cellular proliferation (Ougolkov, Bilim, and Billadeau 2008). Therefore, we decided to analyze the consequences of EZH2 depletion in NKC cells on tumor cell proliferation. Cell counting assay is a very simple method to evaluate cell growth kinetics. 2500 cells were seeded on day 0 and the number of cells was counted subsequently on day 3 and day 6 . On the $6^{\text {th }}$ day, there was a significantly higher number of shRNA control cells than shRNA EZH2 cells (Figure 11A), suggesting that cells grow slower upon depletion of EZH2. Bromodeoxyuridine (BrdU) assay is a widely preferred, efficient method to detect the proliferation of cells. BrdU is a synthetic analog of thymidine and when added on cells gets incorporated into the DNA of replicating cells. The shRNA EZH2 cells displayed lower levels of BrdU incorporation, providing evidence that PDAC cell proliferation reduces significantly upon EZH2 depletion (Figure 11 B).

(A)

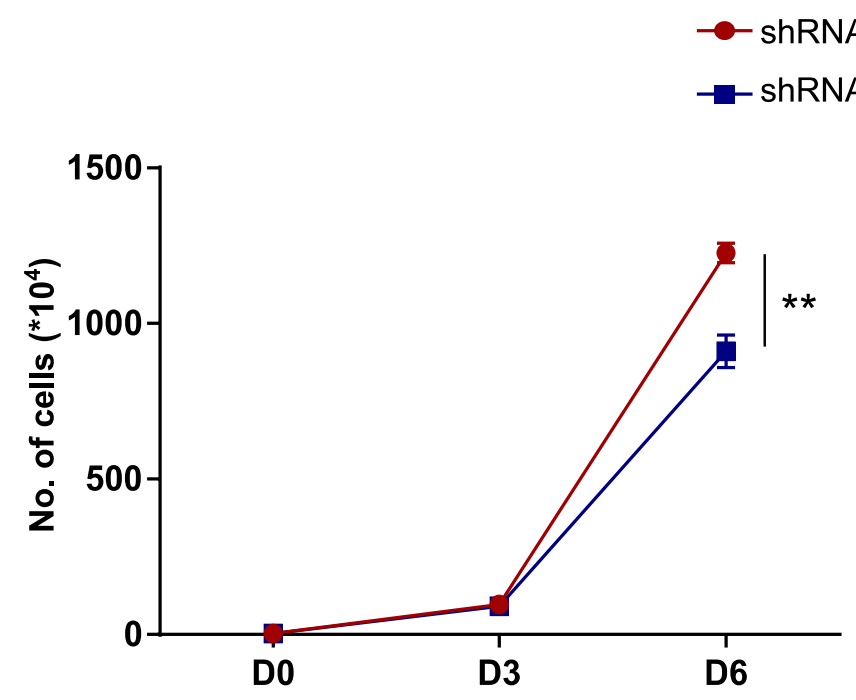

(B)

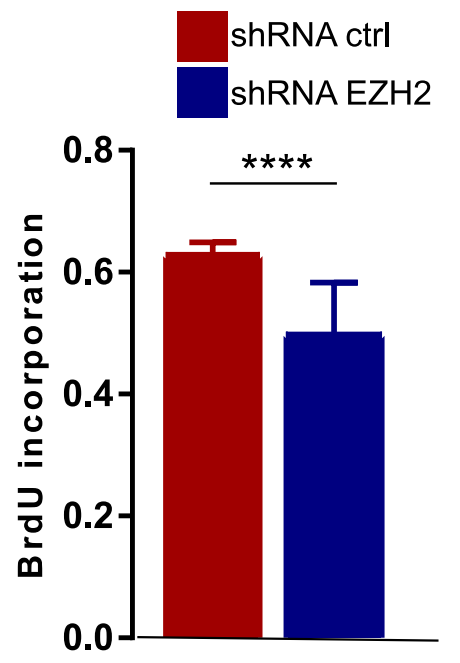

Figure 11: EZH2 depletion reduces cell proliferation in NKC cells.

(A) Graph from cell counting assay depicting the number of cells counted on days 0, 3 and 6 in the indicated cells. (B) Bar graph from BrdU cell proliferation assay showing $\mathrm{BrdU}$ incorporation in NKC cells in the presence and absence of EZH2. Values represent mean \pm SD from 3 independent experiments. Significance was determined by a two-tailed unpaired student's t-test. 


\subsubsection{EZH2 depletion reduces stemness properties in NKC cells}

Another important property of cancer cells is anchorage independent growth (Hanahan and Weinberg 2011) which can be assessed in vitro by colony formation (also known as soft agar assay) assay. This assay evaluates the ability of high proliferating tumor cells to form colonies on the soft agar plate (Borowicz et al. 2014). Our study showed that EZH2 depleted NKC cells have reduced capacity to form colonies on the soft agar compared to the control cells (Figure 12A) confirming that EZH2 deficiency diminishes anchorageindependent growth in NKC cells.

Stemness appears to be a fundamental hallmark of malignancy and tumor cell plasticity (Lathia and Liu 2017). Sphere formation assay is a general in vitro approach to analyze the stemness feature of the cells based on their ability to form spheres when allowed to grow in suspension in a serum-free sphere medium (H. Wang, Paczulla, and Lengerke 2015). In our study, shRNA EZH2 cells formed an overall less number of spheres and relatively smaller spheres compared to the shRNA control cells (Figure 12B), confirming that in the absence of EZH2, NKC cells reduce their stemness potential.

(A)
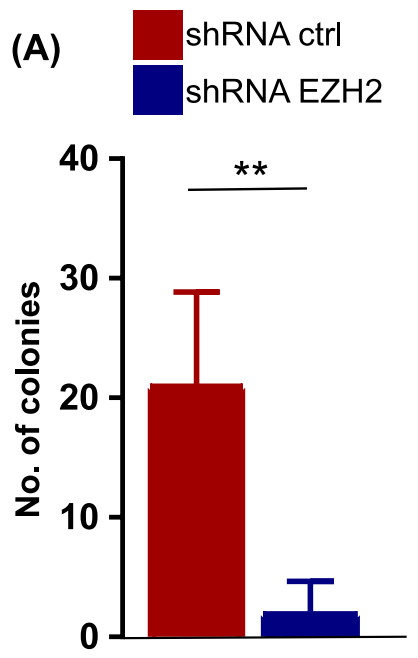

(B)

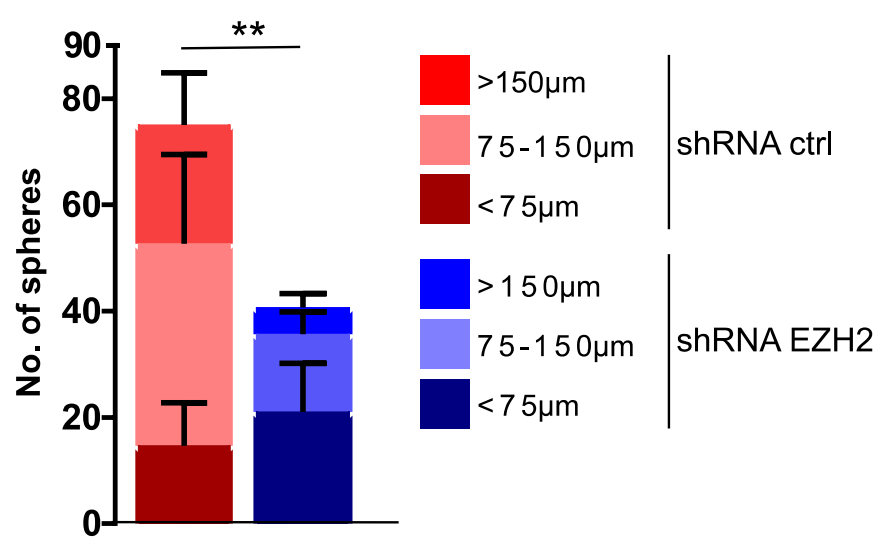

Figure 12: EZH2 deficiency reduces anchorage independent growth and stemness in NKC cells

(A) Bar graph displaying the number of colonies formed in the soft agar in NKC cells in the presence and absence of EZH2. Values represent mean \pm SD from 3 independent experiments. Significance was determined by two-tailed unpaired student's $t$-test. (B) Bar graph illustrating total number and size of spheres in the same cells. Values represent mean \pm SD. Significance was determined by two-way ANOVA. 


\section{RESULTS}

\subsubsection{EZH2 depletion reduces the invasive potential of NKC cells}

Cell invasion is the ability of cancer cells to penetrate through the extracellular matrix which is basically the primary step in the process of metastasis (Zeeshan and Mutahir 2017). Our in vivo data suggests a reduced metastatic potential upon heterozygous Ezh2 depletion (Figure 9). Based on these findings, we performed a Boyden chamber assay which is a commonly used in vitro method to assess tumor cell invasion. The Boyden chamber based invasion assay displayed that upon knockdown of $\mathrm{EZH} 2$, there is a reduction in the capacity of NKC cells to invade (Figure 13).

(A)

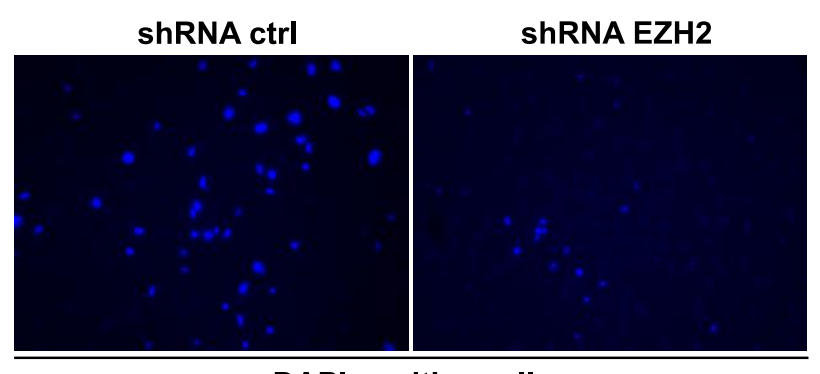

DAPI positive cells $\begin{array}{ll}\text { (B) } & \text { shRNA ctrl } \\ & \text { shRNA EZH2 }\end{array}$

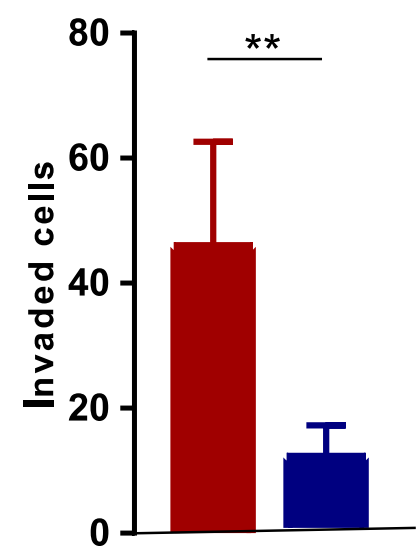

Figure 13: EZH2 knockdown reduces invasive capacity of NKC cells.

(A) Representative image of DAPI stained nuclei demonstrating invaded NKC cells from indicated conditions. (B) Bar graph displaying quantification of invaded cells from Boyden chamber assay shown in A. Values represent mean \pm SD from 3 independent experiments. Significance was determined by two-tailed unpaired student's $t$-test.

Taken together, all the above-mentioned functional assays in PDAC cells indicate that loss of EZH2 significantly diminishes proliferation, anchorage independent growth, stemness and invasive capacity, indicating that EZH2 depletion strongly reduces cellular plasticity and the tumor promoting potential of PDAC cells. 


\section{RESULTS}

\subsection{Mechanistic role of EZH2 in PDAC}

In order to understand the mechanism through which EZH2 mediates regulation of pancreatic plasticity and dedifferentiation, we set up next-generation sequencing approaches in shRNA control and shRNA EZH2 NKC cells. RNAseq was performed to determine differential gene regulation by EZH2 and Chromatin Immunoprecipitation followed by parallel deep sequencing (ChIPseq) identifies the genes directly bound by the methyltransferase (Figure 14). The intersection of information from both assays enables us to identify EZH2dependent gene signatures involved in PDAC progression.

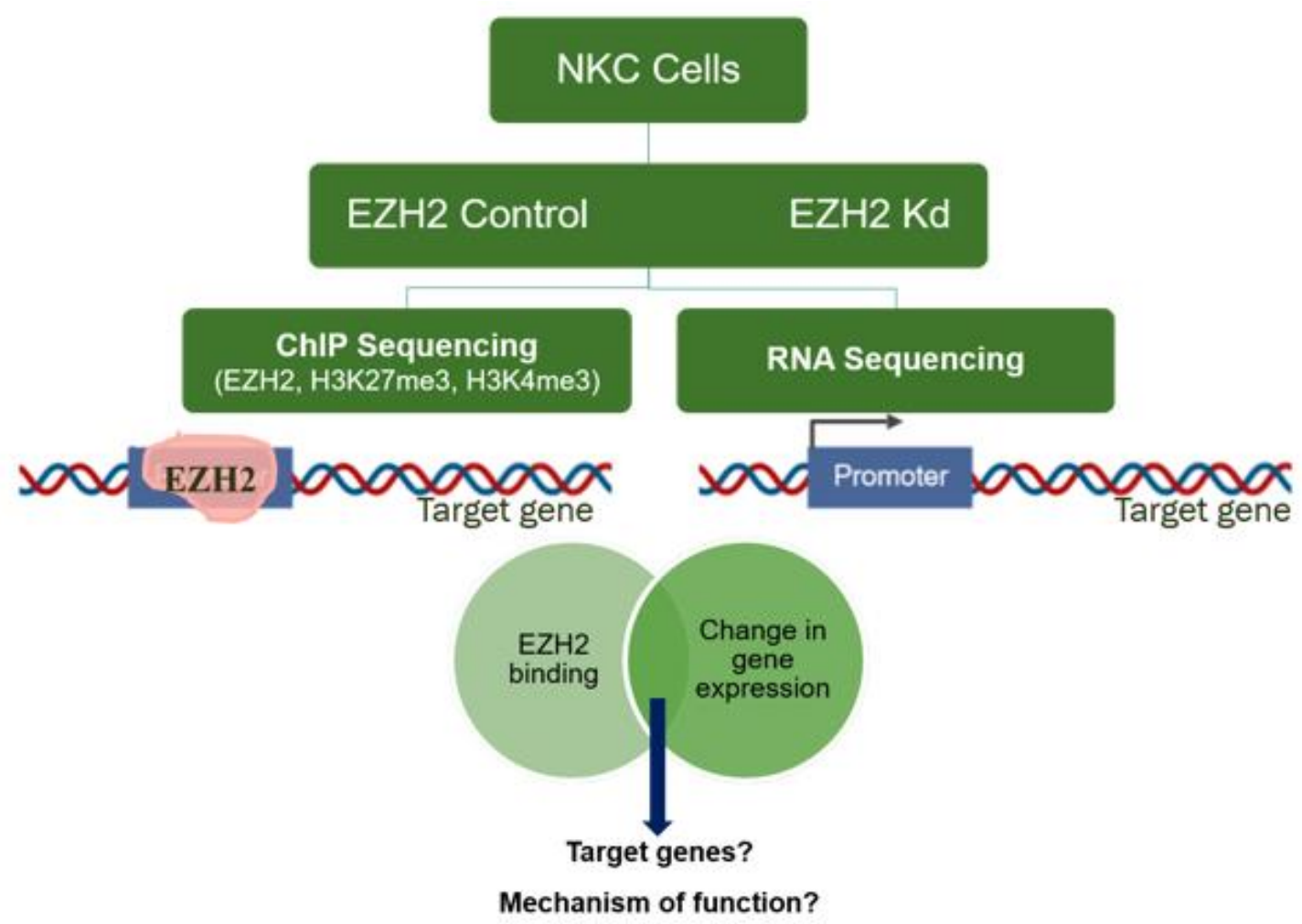

Figure 14: Schematic of the sequencing analysis.

Ovelapping the genes derived from high-throughput ChIP- and RNA- sequencing analysis performed in shRNA control vs shRNA EZH2 NKC cells identify EZH2 direct target genes. 


\section{RESULTS}

\subsubsection{EZH2 regulates genes mediating differentiation and tumor progression in PDAC}

ShRNA control and shRNA EZH2 NKC cells were seeded in triplicates and were subjected to high throughput RNA-seq. Before proceeding with the analysis, we verified the quality of the reads and performed Principal component analysis (PCA) through which we confirmed the similarity between the triplicates of each condition and the differences between the two conditions (Figure 15A). For further analyses, genes with a Fragments Per Kilobase of transcript per Million mapped reads (FPKM) threshold less than 0.2 were eliminated to reduce false positive expressions, which eliminated approximately $50 \%$ of the genes and kept 13,467 genes for downstream analysis.

In order to investigate the pathways affected by EZH2 depletion, we performed Gene set enrichment analysis (GSEA) in control and EZH2 knockdown samples. GSEA is a tool that determines if a predefined gene set coding for a particular pathway is significantly enriched in the given sample (Subramanian et al. 2005). We used the default curated gene sets and examined significantly enriched pathways. While gene signatures related to tumor cell proliferation, migration and metastasis were enriched in shRNA control cells, pathways related to differentiation and apoptosis were enriched upon EZH2 depletion (Figure 15B). In summary, GSEA results strengthened our hypothesis that $\mathrm{EZH} 2$ controls dedifferentiation and metastasis in PDAC. 
(A)

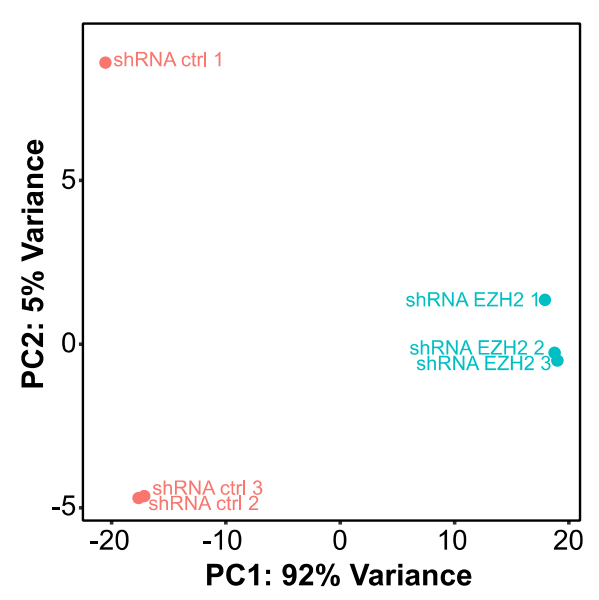

(B)

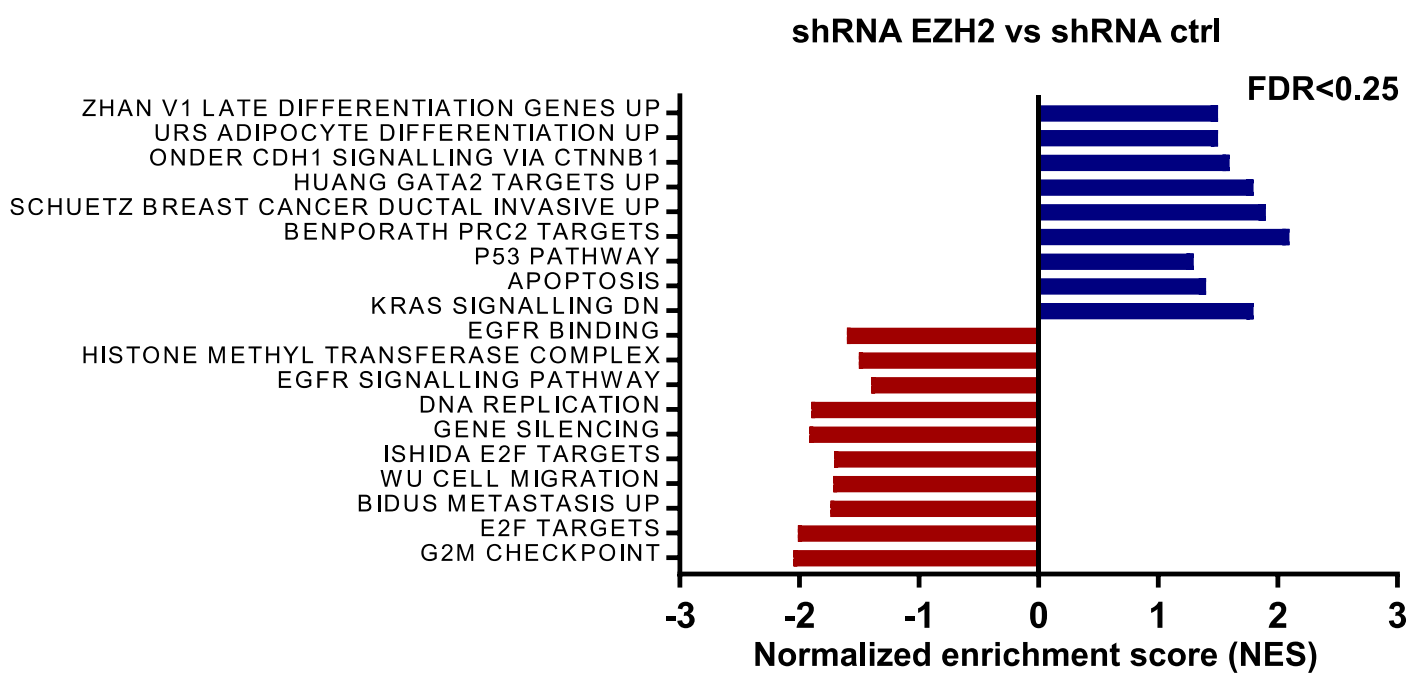

Figure 15: EZH2 deficiency leads to enrichment of pathways related to PDAC progression.

(A) PCA plots from RNA-seq analyses showing the triplicates of shRNA control and shRNA EZH2 clusters. (B) Gene set enrichment analysis (GSEA) performed from RNA-seq analysis displaying pathways positively (blue) and negatively (red) enriched in shRNA EZH2 cells. Significance was determined based on normalized enrichment score (NES) and false discovery rate (FDR) q value.

\subsubsection{EZH2 depletion leads to enrichment of favorable prognosis and differentiation gene sets}

To investigate if EZH2 has any prognostic relevance, we utilized the transcriptomics data from 176 pancreatic cancer patients available in The cancer genome atlas (TCGA). Based on their analyses, 669 genes were 


\section{RESULTS}

classified as unfavorable prognosis genes and 857 genes were identified as favorable prognosis genes in PDAC (Cancer Genome Atlas Research Network et al. 2013). High expression of favorable genes at diagnosis indicated better overall survival whereas increased expression of unfavorable genes indicated lower survival of PDAC patients. We performed GSEA on our genome-wide expression data using these prognostic gene sets. There was a positive enrichment of genes associated with favorable prognosis in the context of $\mathrm{EZH} 2$ deficiency, supporting that depleting $\mathrm{EZH} 2$ enables a better prognosis (Figure 16A).

(A)

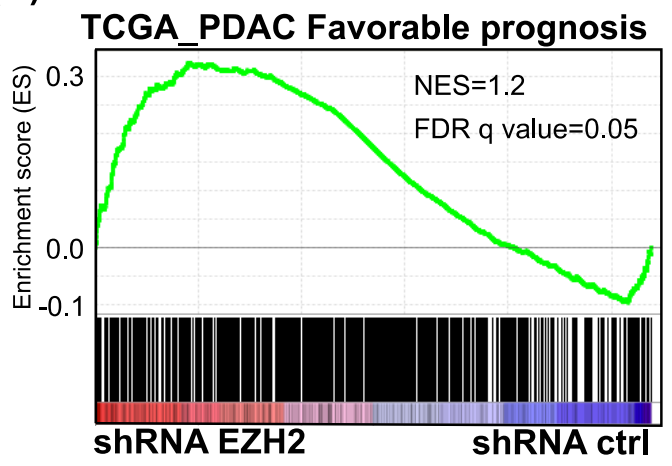

(B)

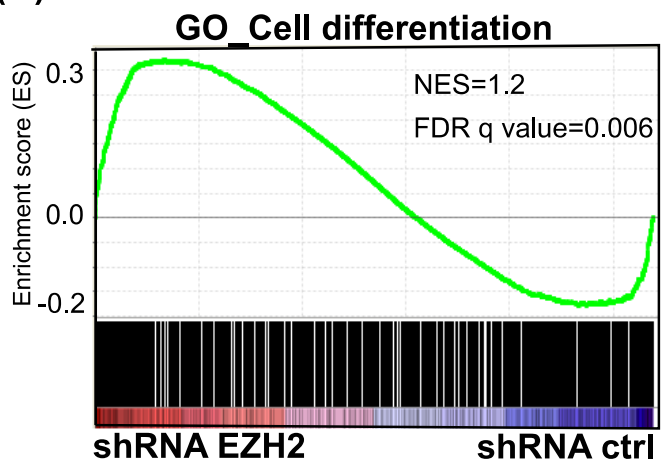

(C)

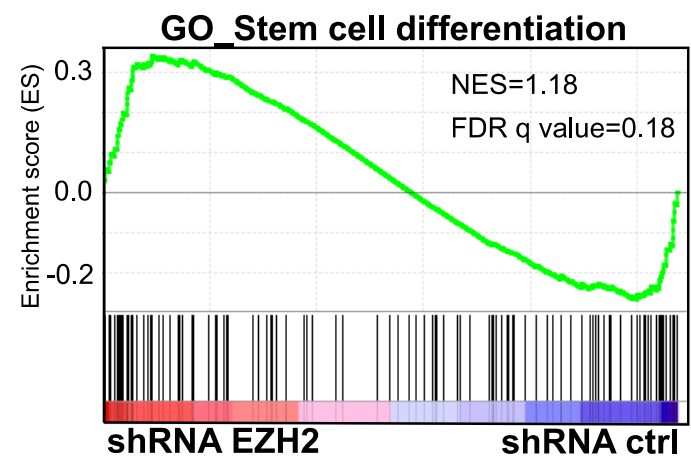

Figure 16: EZH2 depletion mediates better prognosis and cell differentiation programs.

GSEA performed from RNA-seq analysis illustrating enrichment of (A) PDAC favorable prognosis gene set (B) cell differentiation gene set and (C) stem cell differentiation gene set upon EZH2 depletion. Significance was determined based on NES and FDR q value. 
Our findings accomplished in in vivo models and functional assays link EZH2 to PDAC dedifferentiation. In order to explore if $\mathrm{EZH} 2$ regulates gene signatures related to differentiation programs, we performed GSEA using cell- and stem cell- differentiation gene sets. In accordance with our findings in transgenic mice and PDAC cells, we observed an induction of gene signatures associated with cellular differentiation upon EZH2 depletion (Figure 16 B\&C). These observations argue that EZH2 regulates PDAC plasticity by repressing programs required to maintain a differentiated state.

\subsubsection{Classical subtype PDAC genes are positively enriched upon EZH2 depletion}

So far, our data suggest that EZH2 activity is associated with an aggressive PDAC phenotype, dedifferentiation and an unfavorable prognosis. As these features also characterize certain PDAC subtypes (Collisson et al. 2011; Moffitt et al. 2015), we asked whether EZH2 controls gene signatures associated with a particular molecular PDAC subtype. Two extreme molecular PDAC subtypes have remained consistent in all the studies - the classical and basal-like subtype (P. Bailey et al. 2016; Collisson et al. 2011; Maurer et al. 2019; Moffitt et al. 2015; Puleo et al. 2018).

In one of the latest works with regard to PDAC subtypes performed by Puleo et al., they considered all previous subtyping studies and defined two important PDAC subtypes in the epithelial compartment of the tumor - pure classical and basal-like subtypes (Puleo et al. 2018). While the basal-like subtype exhibits higher tumor grade and poor survival, the pure classical subtype is associated with a more differentiated phenotype and exhibits a better prognosis. To evaluate if $\mathrm{EZH} 2$ is involved in the regulation of gene signatures defining pure classical vs basal-like subtypes, we intersected our RNA-seq data with Puleo et al. defined gene sets. Interestingly, we found a positive enrichment of pure classical gene signatures and negative enrichment of basal-like gene signatures in shRNA EZH2 samples (Figure 17 A-D). 


\section{RESULTS}

(A)

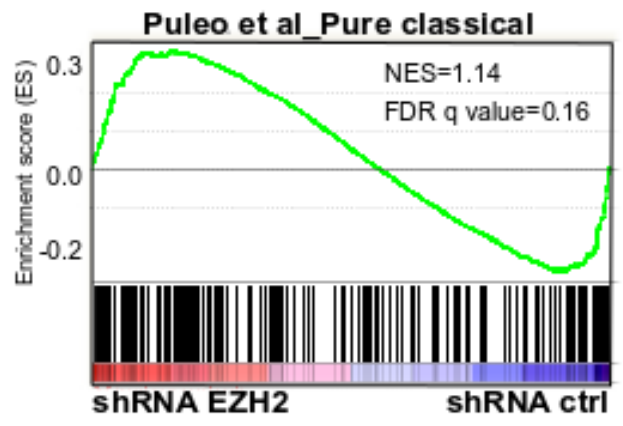

(B)
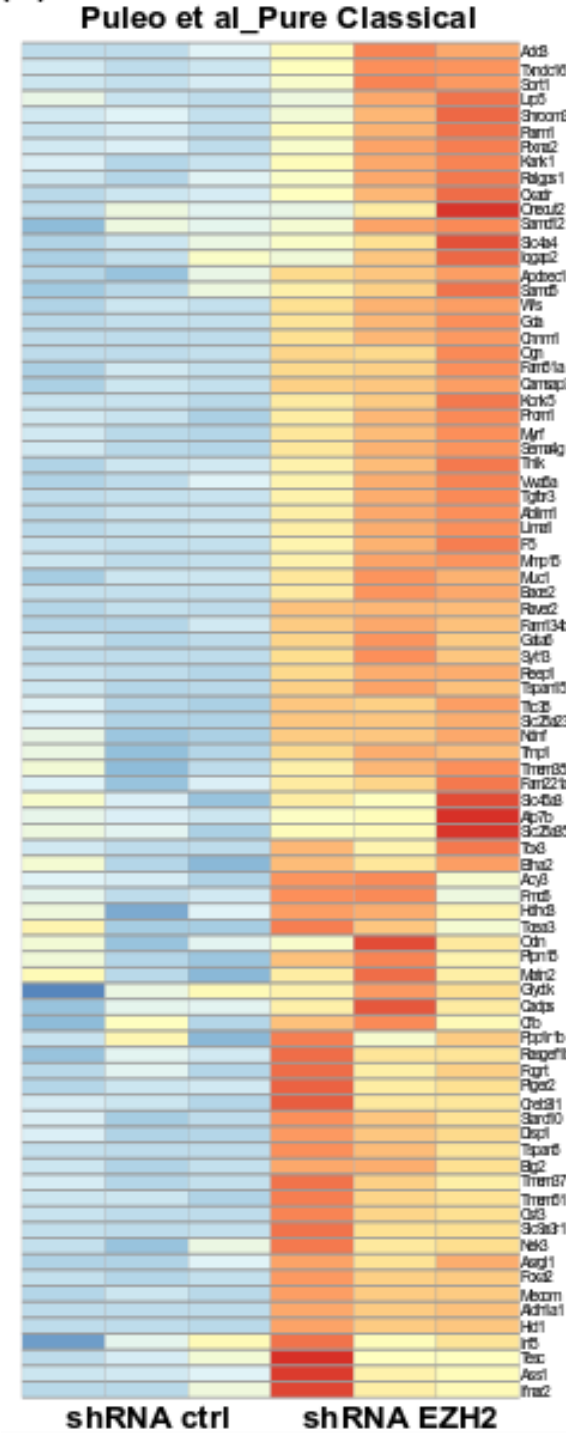

(C)

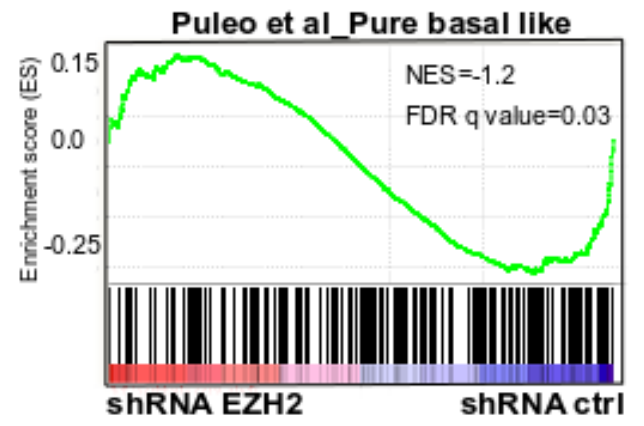

(D)
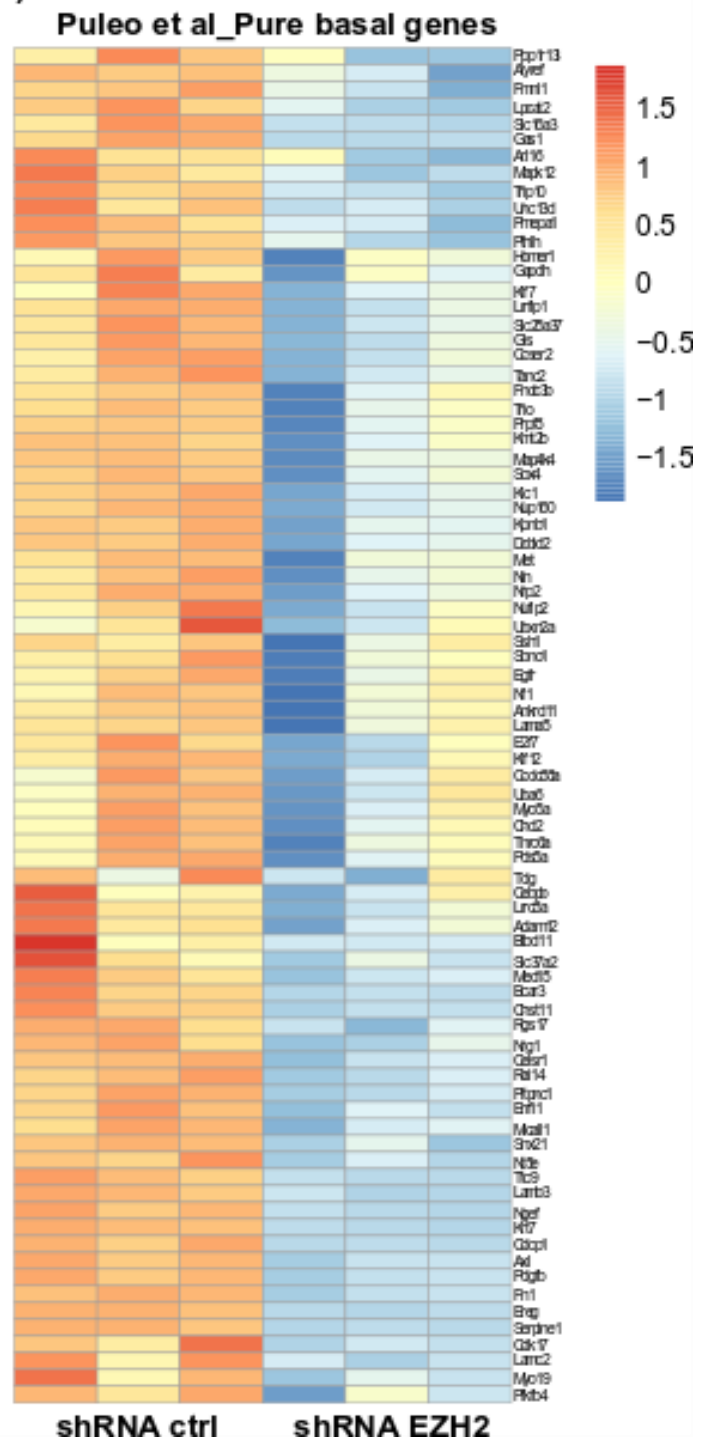

Figure 17: EZH2 knockdown leads to positive enrichment of classical PDAC- and negative enrichment of basal-like PDAC subtype gene signatures.

Analysis of RNA-seq (A) GSEA plot and (B) Heatmap illustrating positive enrichment of Pure classical gene signatures (Puleo et al. 2018) upon EZH2 knockdown. (C) GSEA plot and (D) Heatmap illustrating negative enrichment of basal-like gene signatures (Puleo et al. 2018) upon EZH2 knockdown. Significance was determined based on NES and FDR q value. 
Importantly, molecular PDAC subtypes not only display distinct histological features and prognostic implications, they also exhibit diverse metabolic profiles. Indeed, Daemen et al. have identified two extreme metabolic profiles which strongly associate with the classical and basal-like subtypes (Daemen et al. 2015). While the lipogenic subtype that consists of genes associated with lipogenesis is strongly associated with classical PDAC, the glycolytic subtype which comprises genes involved in glycolysis correlates with basal PDAC gene signatures (Daemen et al. 2015). In line with the aforementioned enrichment of classical gene signatures in shEZH2 cells, GSEA using these metabolic gene sets revealed that lipogenic gene signatures are enriched in the context of EZH2 deficiency (Figure 18). This data further strengthens the observation that EZH2 depletion is associated with a less aggressive classical PDAC subtype.

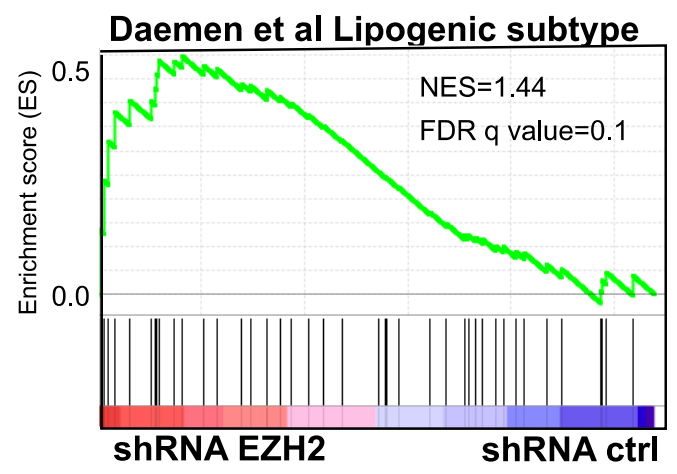

Figure 18: EZH2 knockdown leads to enrichment of lipogenic subtype gene signatures.

Analysis of RNA-seq: GSEA plot depicting enrichment of lipogenic gene signatures in shRNA EZH2 condition. Significance was determined based on NES and FDR q value.

\subsubsection{EZH2 binds largely on TSS/promoter sites of its target genes}

The transcriptomics data supported the hypothesis that EZH2 regulates differentiation-associated gene signatures and pathways. To further elucidate the mechanistic background of oncogenic EZH2 activity in PDAC and to identify target genes directly bound by EZH2, we performed genome-wide binding analysis by ChIP-seq. To this end, shRNA control and shRNA EZH2 NKC cells 


\section{RESULTS}

were seeded in triplicates and subjected to ChIP-seq. ChIP-seq with an antibody recognizing EZH2 was performed in shRNA control cells to identify the genes occupied by EZH2. Firstly, we analyzed the location on the genome where EZH2 majorly binds. We assessed the enrichment of EZH2 binding at different genomic regions like promoter, introns, exon etc. using Cis-regulatory Element Annotation System (CEAS). CEAS is a tool that enables the visualization of relative ChIP enrichment at important genomic locations compared to the whole genome (Shin et al. 2009). The CEAS analysis of EZH2 ChIP-seq revealed that most of the EZH2 binding regions were confined to the TSS/Promoter of the genes $(17.1 \%)$ compared to whole-genome $(1.1 \%)$ (Figure 19A). Additionally, GREAT analysis also validated the genome-wide EZH2 occupancy predominantly near the TSS of target genes (Figure 19B). 
(A)

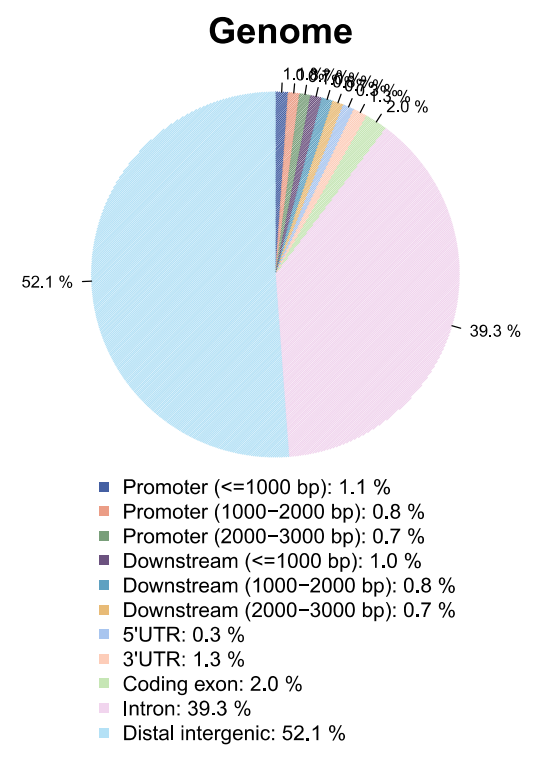

EZH2 ChIP

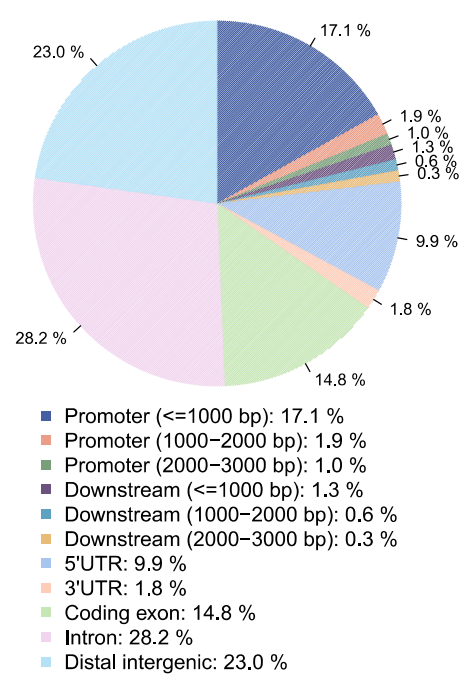

(B)

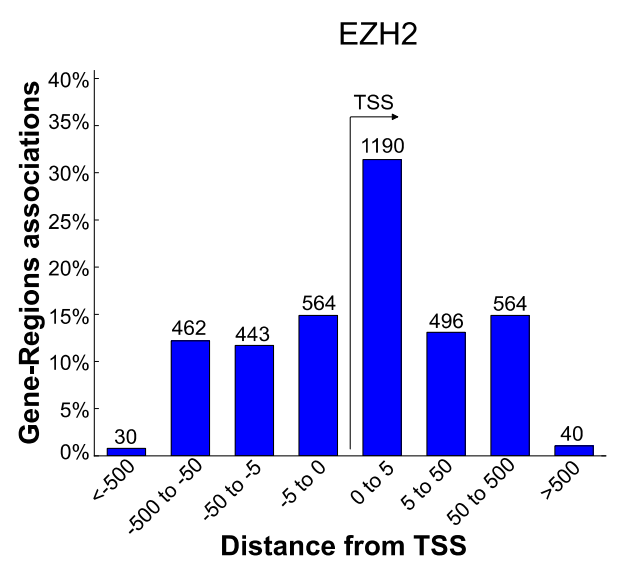

Figure 19: EZH2 largely occupies the promoter region of its target genes.

(A) Cis-regulatory Element Annotation System (CEAS) analysis of EZH2 ChIP-seq showing relative enrichment of EZH2 on promoters and gene bodies compared to the whole genome. (B) Genomic Regions Enrichment of Annotations Tool (GREAT) analysis of ChIP-seq data revealing that EZH2 largely binds around the TSS of the gene.

\subsubsection{EZH2-deficiency fosters a shift in histone modifications at EZH2 target genes}

To study EZH2-dependent histone modifications, EZH2 ChIP-seq was accompanied by ChIP-seq analysis for H3K27me3 and H3K4me3 in the presence and absence of EZH2. Principal component analysis (PCA) in H3K27me3 and H3K4me3 ChIP-seq confirmed the similarity between the 


\section{RESULTS}

triplicates of each condition and the differences between the two conditions (Figure 20).
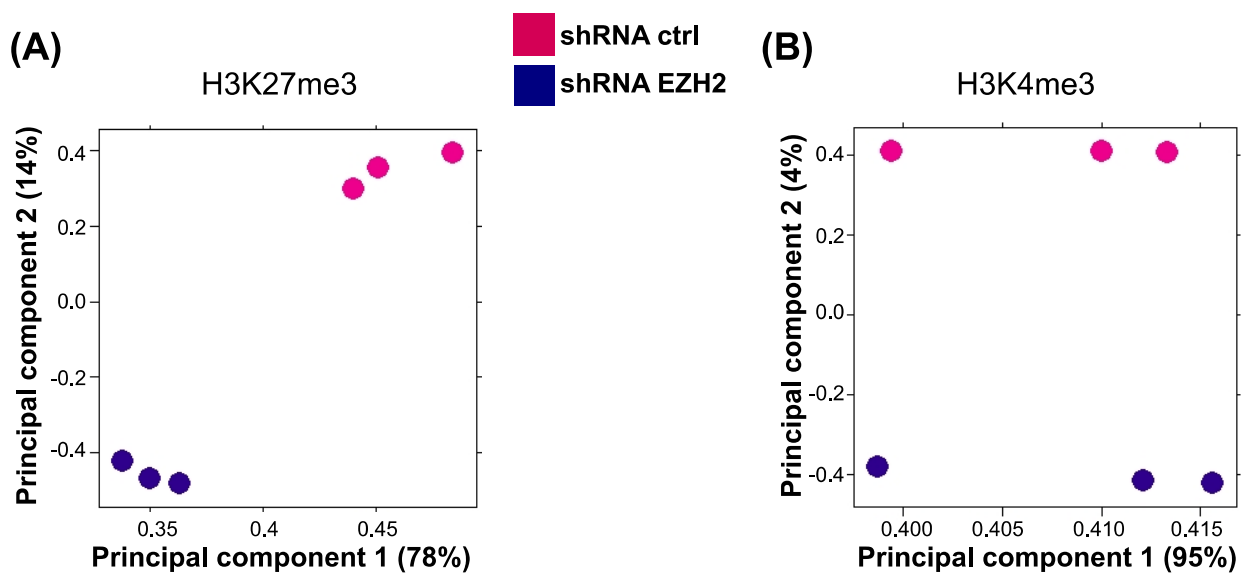

Figure 20: PCA plots of histone marks from ChIP sequencing analysis

PCA plots from ChIP-seq analysis of (A) H3K27me3 and (B) H3K4me3 illustrating distinct clusters of shRNA control and shRNA EZH2 triplicates.

Subsequently, we developed heatmaps to study the general trends in genomewide $\mathrm{EZH} 2$ binding and subsequent enrichment of histone modifications. Figure $21 \mathrm{~A}$ displays the heatmap profile of $\mathrm{EZH} 2$ binding on the shRNA control genome. Consistent with its function as a transcriptional repressor, EZH2bound regions were enriched for the repressive H3K27me3 mark, which was decreased in EZH2 knockdown cells (Figure 21B). In contrast, H3K4me3, which is a transcriptionally active mark (Hemming et al. 2016; P. Wang et al. 2018), displayed increased enrichment in these EZH2 depleted regions (Figure $21 \mathrm{C})$. 
(A)

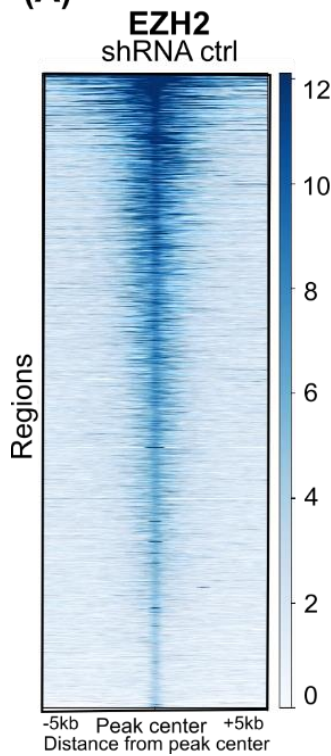

(B)

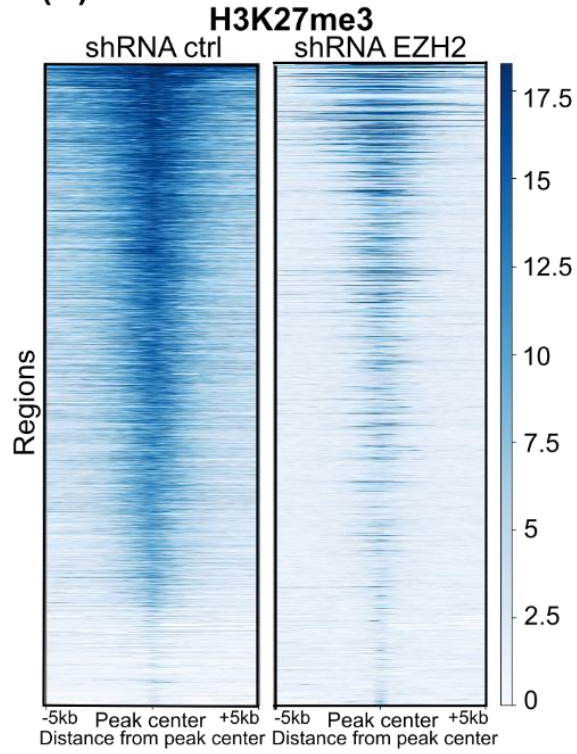

(C)

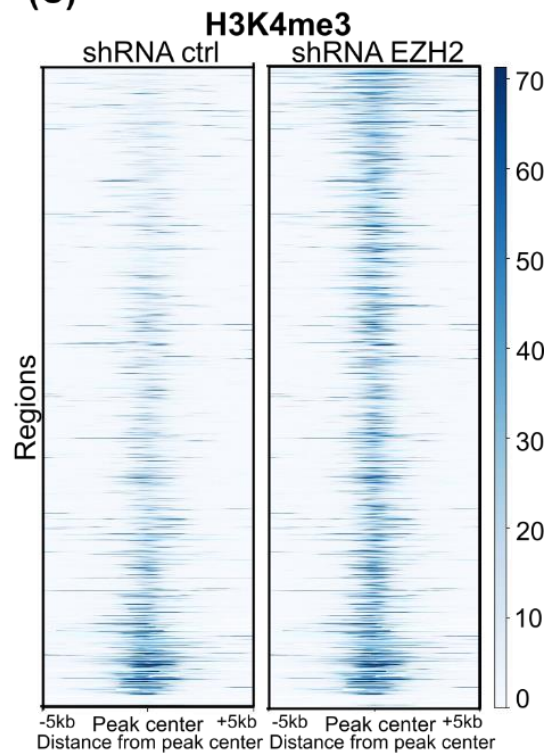

Figure 21: Loss of EZH2 binding leads to decrease in $\mathrm{H} 3 \mathrm{~K} 27 \mathrm{me} 3$ and increase in H3K4me3 enrichment.

Heatmaps from ChIP-seq data in NKC cells depicting (A) EZH2 binding on the genome. (B) H3K27me3 occupancy and (C) H3K4me3 occupancy on EZH2 bound regions in indicated conditions.

\subsubsection{EZH2 targets differentiation-associated genes}

In order to identify direct EZH2 targets genes, we combined the output of our ChIP- and RNA-seq data. With the ChIP-seq data, we performed differential binding (Diffbind) analysis to identify differentially occupied regions in shRNA EZH2 compared to shRNA control NKC cells. Furthermore, we performed Genomic Regions Enrichment of Annotations Tool (GREAT) analysis to identify genes associated with regions identified by Diffbind analysis.

To obtain direct EZH2 binding targets, we focused on genes that i) showed EZH2 binding on shRNA control samples, ii) significantly lost H3K27me3 enrichment ( $\log 2 \mathrm{FC}>2, \mathrm{q}<0.1$ ) and iii) gained H3K4me3 occupancy ( $\log 2 \mathrm{FC}$ $>0.5, q<0.1)$ upon EZH2 knockdown. This strategy led to the identification of 965 target genes (Figure 22A) which are bound and potentially repressed by the histone methyltransferase. We additionally performed CRISPR/Cas9 mediated knockout of EZH2 in NKC cells (shown in appendix, figure 35) and further subjected CRISPR/Cas9 control vs CRISPR/Cas9 EZH2 NKC cells to RNA-seq to identify the genes regulated by EZH2. Subsequently, 965 target 
genes identified by ChIP-seq were intersected with genes that were found to be upregulated upon shRNA- $(\log 2 \mathrm{FC}>0.5, \mathrm{q}<0.1)$ and CRISPR/Cas9 $(\log 2 \mathrm{FC}>$ $0.5, \mathrm{q}<0.1)$ mediated depletion of EZH2 in the RNA-seq. We identified 47 final direct target genes (Figure 22B), whose TSS region is bound by EZH2 and meanwhile expression is increased in the context of EZH2 depletion. Consistent with our observations in phenotypic and functional data, Gene ontology (GO) analysis of these 47 genes revealed pathways related to development and differentiation (Figure 22C).

(A)

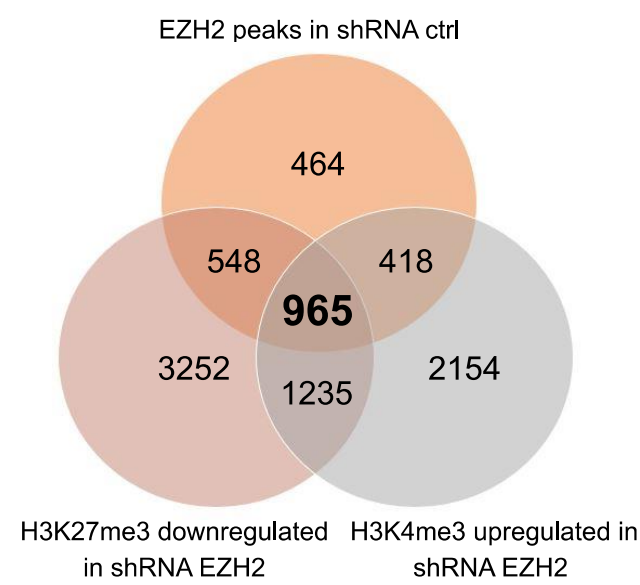

(B) $\begin{array}{cc}\text { direct } \mathrm{EZH} 2 \text { targets } & \begin{array}{c}\text { Genes upregulated in } \\ \mathrm{n}=965\end{array} \\ \text { shRNA EZH2 }\end{array}$

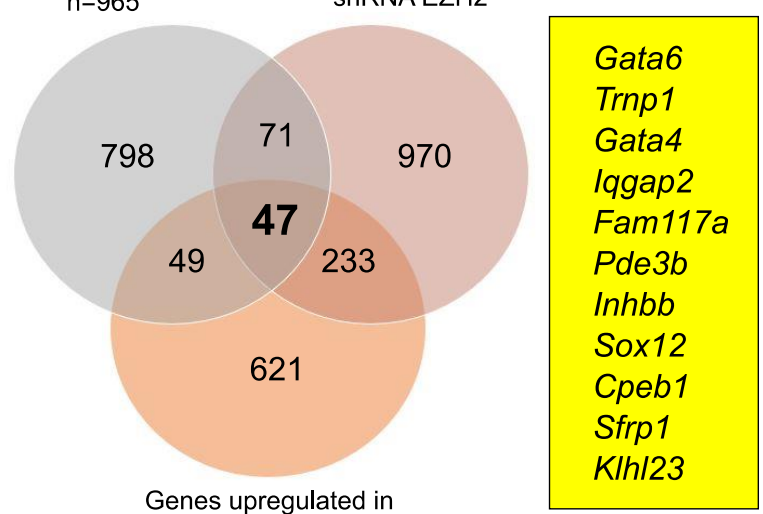

CRISPR/Cas9 EZH2

(C)

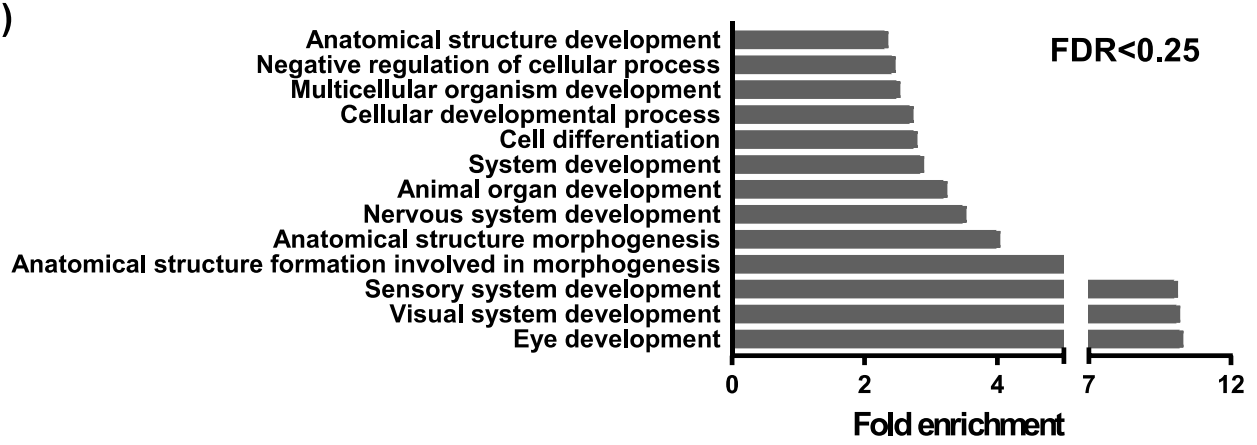

Figure 22: Direct target genes of EZH2 mediate developmental and cell differentiation related programs.

(A) Venn diagram showing the intersection of genes fulfilling the following criteria: bound by EZH2, decreased H3K27me3 enrichment in shRNA EZH2 samples (log2FC $>2, \mathrm{q}<0.1$ ) and increased H3K4me3 enrichment in shRNA EZH2 samples (log2FC > $0.5, \mathrm{q}<0.1$ ) as identified by ChIP-seq. (B) Venn diagram showing intersection of direct EZH2 target genes from $A$ with genes upregulated upon shRNA- $(\log 2 F C>0.5, q<$ $0.1)$ and CRISPR/Cas9- $(\log 2 \mathrm{FC}>0.5, \mathrm{q}<0.1)$ mediated EZH2 depletion. Box indicates an excerpt of the final 47 target genes. (C) GO gene enrichment analysis depicts pathways associated with $47 \mathrm{EZH} 2$ target genes. $(\mathrm{FDR}<0.25)$ 
Out of the 47 direct EZH2 targets we selected a set of eleven genes with potential implication in PDAC biology. The ChIP-seq based binding profiles were viewed using an integrated genome viewer (IGV), which is a widely used visualization tool for NGS data (Robinson et al. 2011; Thorvaldsdóttir, Robinson, and Mesirov 2013). The IGV profile of 11 selected EZH2 target genes depicted in figure 23 displays the $\mathrm{EZH} 2$ binding and associated changes in occupancy of histone marks on TSS of the genes in the presence and absence of EZH2.

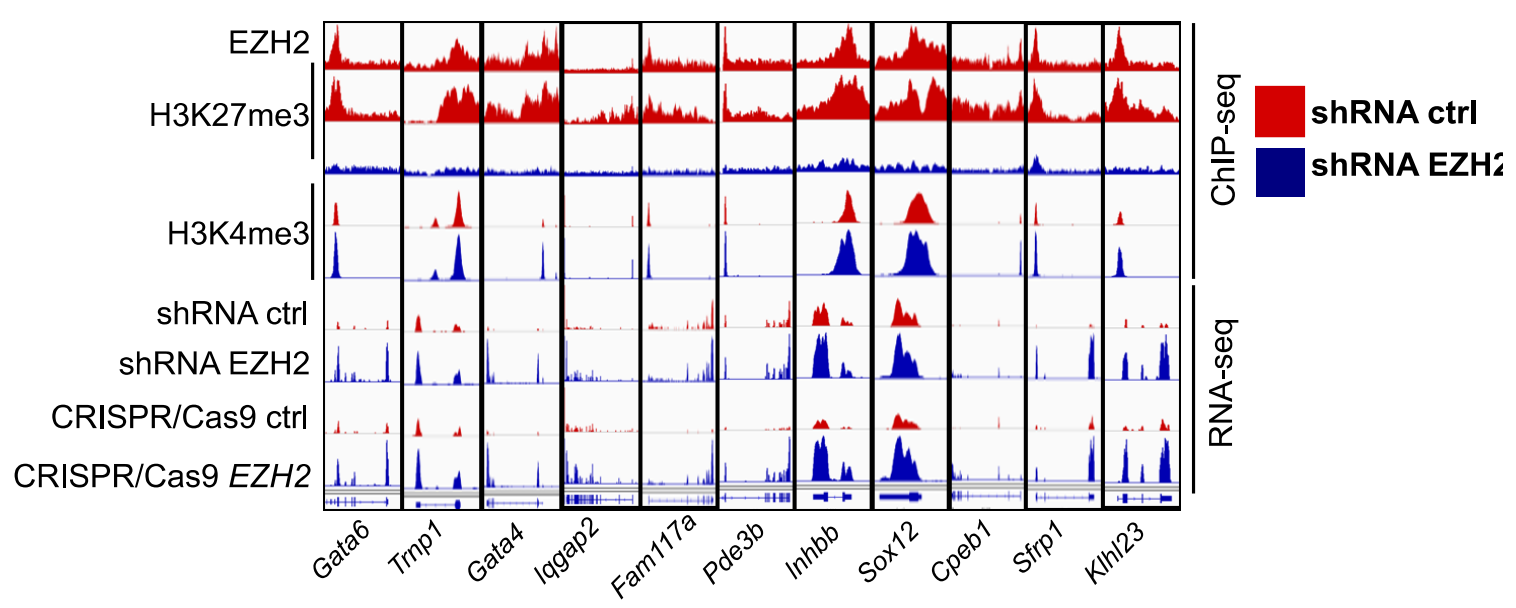

Figure 23: IGV profile of selected $11 \mathrm{EZH2}$ direct target genes.

11 genes with potential implications in PDAC were selected from the 47 direct EZH2 target genes. The figure depicts the Integrated genome viewer (IGV) profiles of the finally selected $11 \mathrm{EZH} 2$ target genes.

\subsubsection{EZH2 target gene expression validation}

The selected EZH2 target genes were further validated in independent experiments. We performed expression analysis of these target genes in shRNA control vs shRNA EZH2 NKC cells, CRISPR/Cas9 control vs CRISPR/Cas9 EZH2 NKC cells as well as upon treatment with the EZH2 inhibitor Tazemetostat (EPZ6438). These analyses revealed that the expression of all eleven genes increases significantly upon genetic or pharmacological inhibition of the histone methyltransferase, although to different magnitudes in the different conditions (Figure 24). The western blot confirming successful reduction of H3K27me3 levels upon Tazemetostat (EPZ6438) treatment is shown later in Figure 27F. 


\section{RESULTS}

(A)

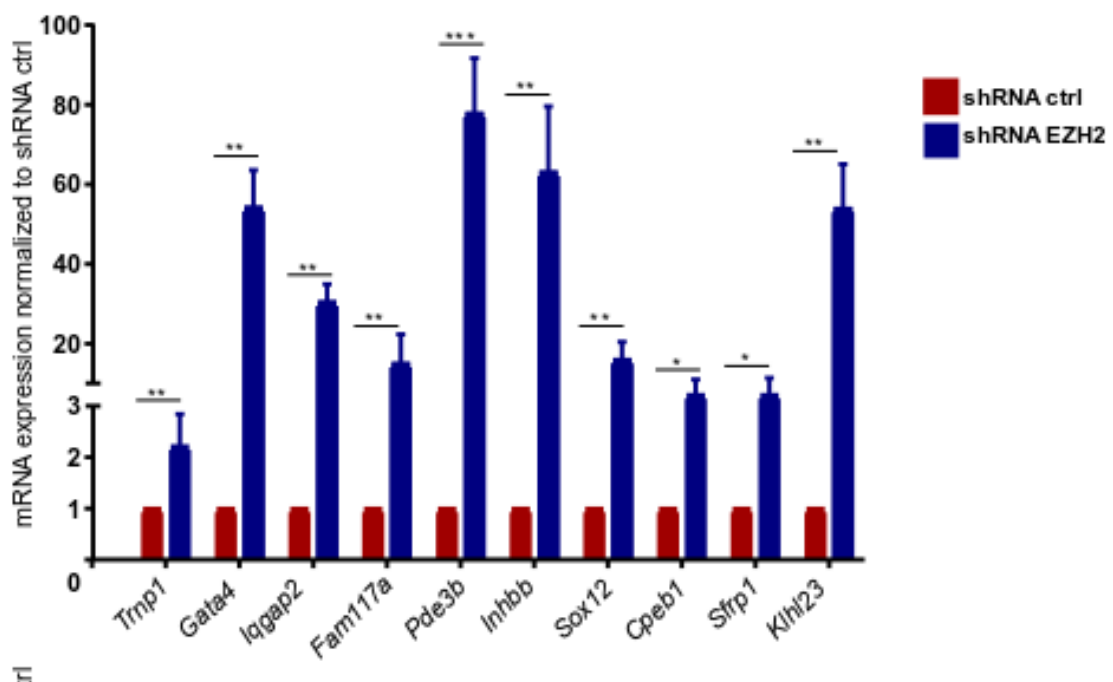

(B)

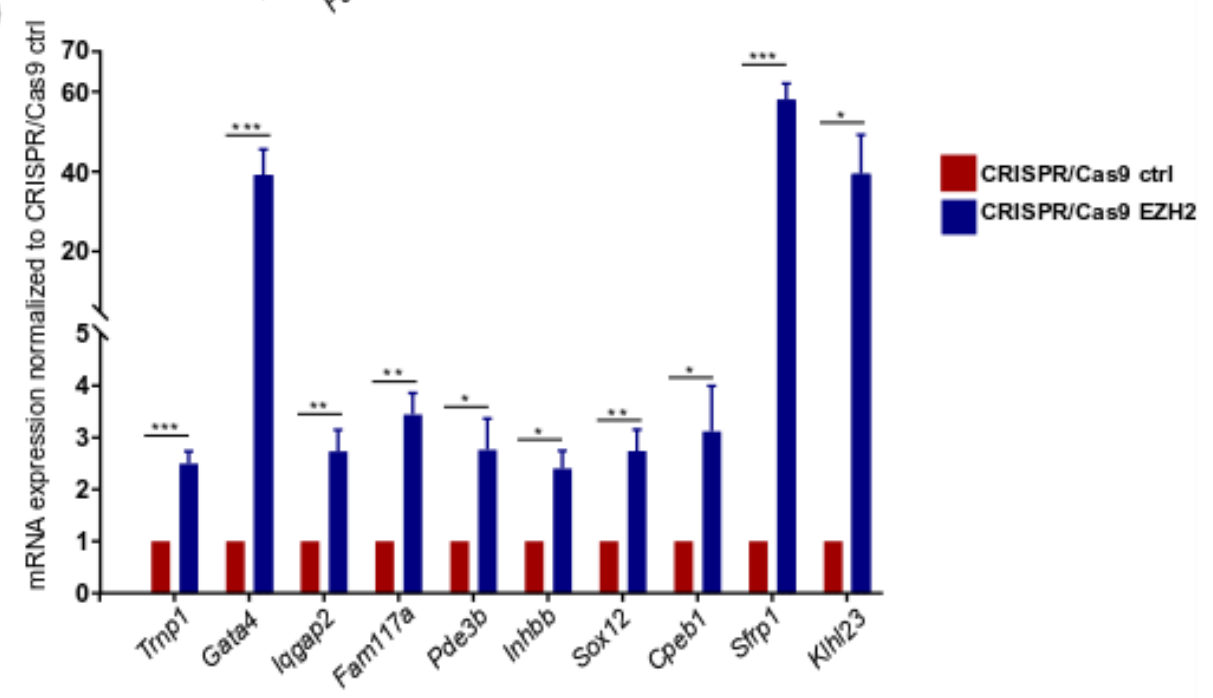

(C)

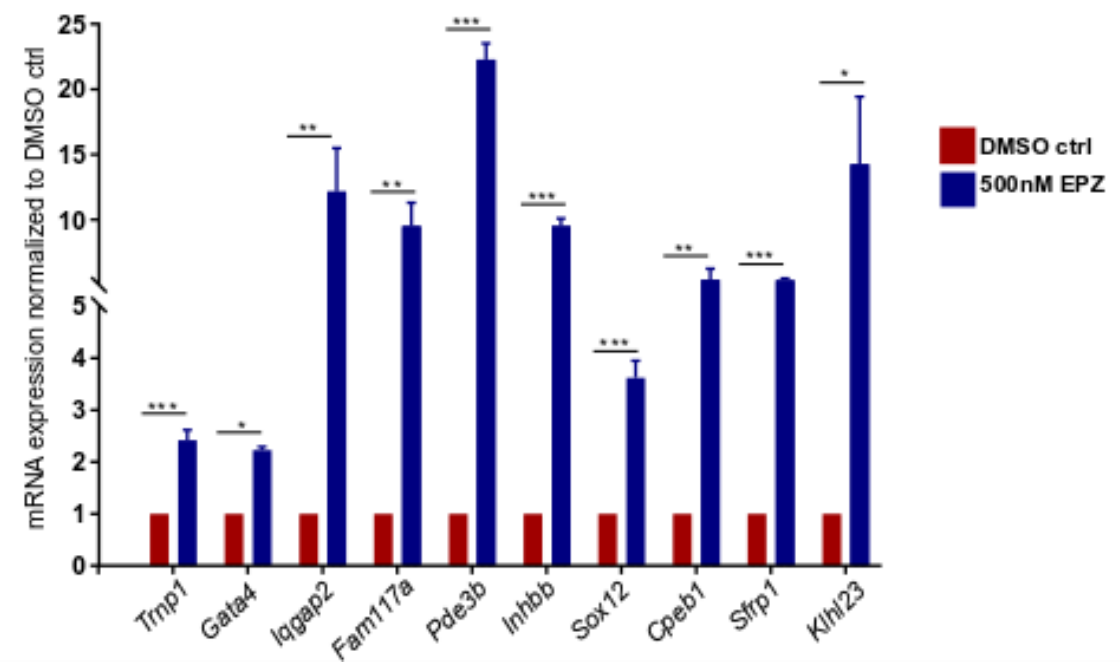

Figure 24: Expression of direct EZH2 target genes increases upon EZH2 depletion.

qPCR validating the expression of the selected $11 \mathrm{EZH} 2$ target genes in (A) shRNA control vs shRNA EZH2 NKC cells (B) CRISPR/Cas9 control vs CRISPR/Cas9 EZH2 NKC cells (C) DMSO treated vs 500nM Tazemetostat (EPZ6438) (for 72 hours) treated NKC cells. Two-tailed student's $t$ test was used to determine the significance. 
Furthermore, we designed specific primers covering the TSS/promoter of the the selected target genes where a peak for EZH2 was detected. Also, for the negative control, we designed primers across the intragenic region of the gene where EZH2 occupancy was not observed. The individual ChIP qPCR analysis validated EZH2 occupancy as well as EZH2-dependent H3K27me3 and H3K4me3 occupancies specifically on the TSS/promoter of the target genes.
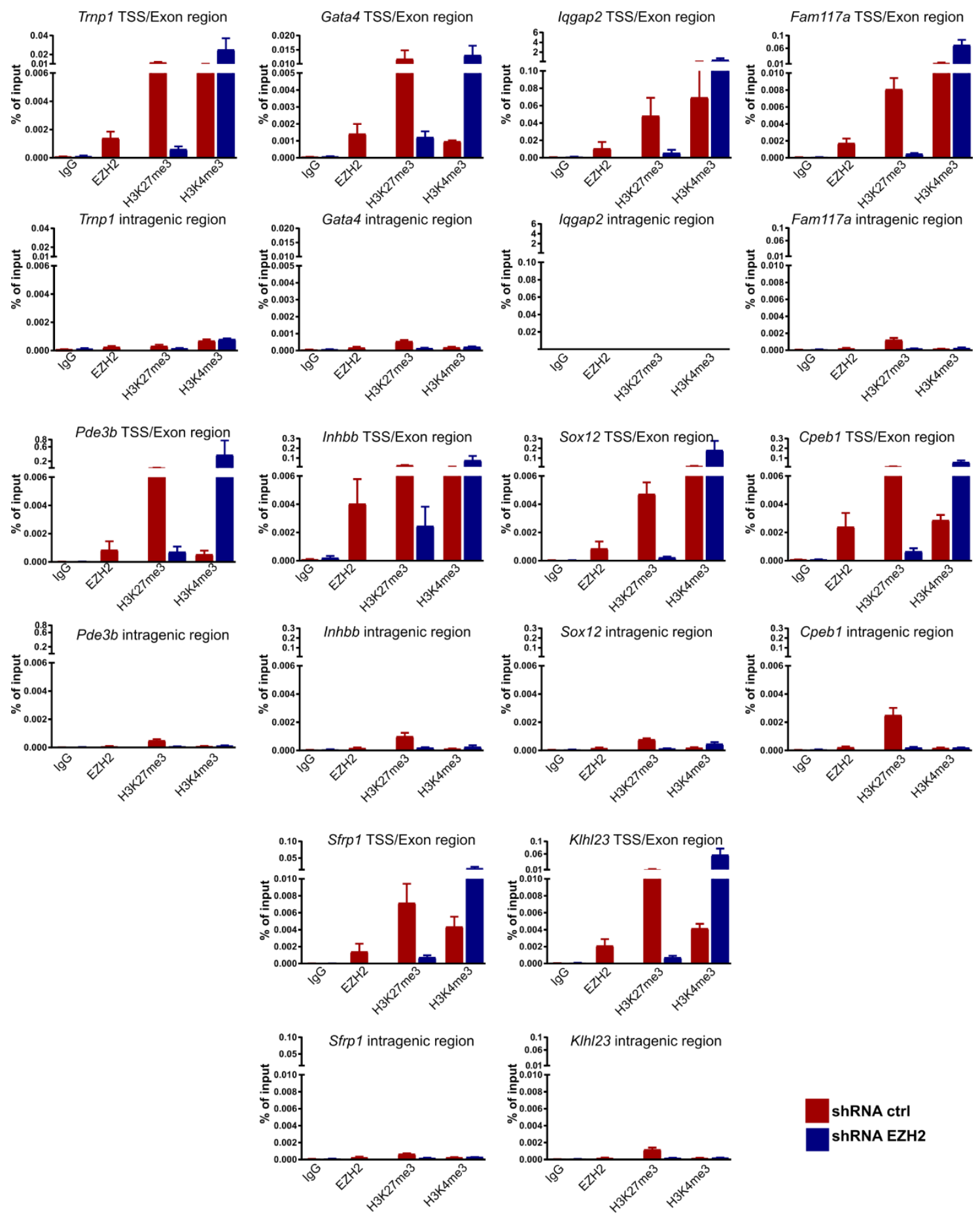

ShRNA ctrl

shRNA EZH2 
Figure 25: EZH2 binds to TSS of its target genes and mediates promoter specific histone modifications.

qRT-PCR following EZH2- and histone modification ChIP analysis utilizing primers embracing the TSS/promoter region and intragenic region (negative control) of selected genes for validation as a direct EZH2 target gene.

\subsection{EZH2-GATA6 axis in PDAC}

\subsubsection{EZH2 targets epithelial transcription factor Gata6}

One of the well-regulated and interesting EZH2 target genes was the transcription factor GATA6. GATA6 represents a critical regulator of pancreatic development and endodermal lineage differentiation (C. Y. Chia et al. 2019; Lorberbaum and Sussel 2017; Tiyaboonchai et al. 2017). Its expression in various cancers including pancreatic cancer is linked to blocking pancreatic carcinogenesis and PDAC progression. GATA6 loss is associated with reduced differentiation and increased metastasis in PDAC (Martinelli et al. 2017). Further evidence states that GATA6 activates transcriptional programs related to epithelial differentiation (Hermann et al. 2014; Martinelli et al. 2016). Moreover, all the PDAC subtype studies from this decade portray GATA6 as the central regulator of molecular subtype identity. GATA6 proves to be a surrogate marker with high and low GATA6 expression implying classical and basal-like PDAC subtypes, respectively (Aung et al. 2019; P. Bailey et al. 2016; Collisson et al. 2011; O'Kane et al. 2020).

Interestingly, in our ChIP-seq we detected a prominent EZH2 peak around the TSS/promoter of the Gata6 gene. Additionally, we observed a decrease in H3K27me3 enrichment and increase in H3K4me3 occupancy in this region upon the depletion of EZH2. This was in line with our observations in RNA-seq data, where we detected a significantly enhanced upregulation of Gata6 expression upon EZH2 knockdown (Figure 26A). To further validate that EZH2 indeed binds specifically to the promoter of Gata6, we performed individual ChIP experiments. Consistent with our observations in IGV from ChIP-seq, qRT-PCR performed in ChIP samples revealed a significant enrichment of EZH2 binding and its activity (H3K27me3) around the promoter of Gata6. In the context of EZH2 deficiency, binding of EZH2 and $\mathrm{H} 3 \mathrm{~K} 27 \mathrm{me} 3$ reduced while 
H3K4me3 and H3K27ac (active transcription marks) increased (Figure 26B). Moreover, the intragenic regions showed no difference in the enrichment of these marks (Figure 26B) verifying that all the binding and modifications occur specifically around the promoter/TSS of Gata6.

(A)

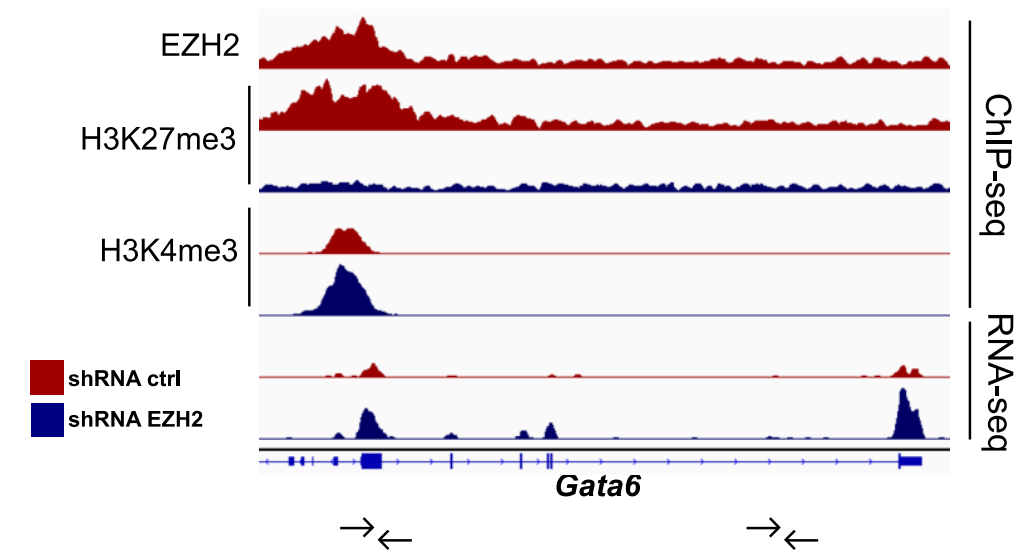

(B)

Gata6 TSS region

Gata6 intragenic region
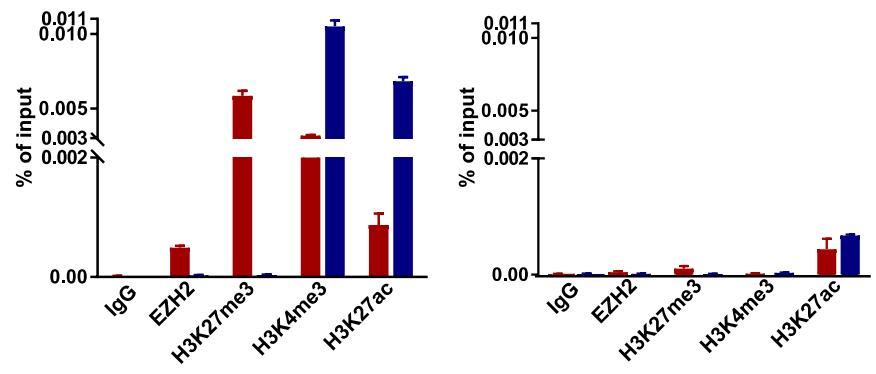

Figure 26: EZH2 binds to TSS of Gata6 and mediates TSS specific histone modifications.

(A) Integrative Genomics Viewer (IGV) profile from ChIP- and RNA-seq at the Gata6 gene locus in NKC cells showing EZH2 binding and enrichment of H3K27me3 and H3K4me3 at the Gata6 gene in indicated conditions. Arrows indicate location of primers designed for individual ChIP validation studies shown in B. (B) qRT-PCR following EZH2- and histone modification ChIP analysis utilizing primers embracing the TSS/promoter region and intragenic region (negative control) of Gata6 for validation as an $\mathrm{EZH} 2$ target gene. 


\subsubsection{EZH2 depletion significantly increases Gata6 expression in murine PDAC cells}

We further aimed to scrutinize if EZH2 dependent Gata6 regulation could be validated in vitro in PDAC cell lines. Therefore, we investigated the EZH2Gata6 axis in various cell lines utilizing different genetic as well as pharmacological approaches to manipulate EZH2 expression and activity. Gata6 expression was upregulated both at the RNA and protein level upon shRNA- and CRISPR/Cas9-mediated EZH2 depletion (Fgure 27 A-D). Importantly, re-expression of EZH2 in EZH2 deficient NKC cells rescued Gata6 upregulation (Figure $27 \mathrm{C} \& \mathrm{D}$ ). Moreover, Gata6 transcription was induced when NKC cells were treated with Tazemetostat (EPZ6438) (Figure $27 \mathrm{E}$ ), which specifically inhibits the histone methyltransferase activity of EZH2 (Italiano et al. 2018; McGrath and Trojer 2015). The western blot shows the reduction in H3K27me3 levels upon treating NKC cells with Tazemetostat (EPZ6438), confirming that the inhibitor indeed reduced the enzymatic activity of EZH2 (Figure $27 \mathrm{~F}$ ). This suggests that blockade of EZH2 activity is sufficient to re-install Gata6 expression. Furthermore, we analyzed Gata6 expression in primary PDAC cells derived from caNFATC1;Kras ${ }^{G 12 D}$ (NKC) and $E Z H 2^{\text {fll }}$;caNFATc1;KrasG12D (ENKC) mice. As expected, there was an upregulation of Gata6 mRNA and protein levels in ENKC cells compared to NKC cells (Figure $27 \mathrm{G \& H}$ ), further confirming Gata6 as a transcriptional EZH2 target. To understand if the EZH2-Gata6 axis is restricted to PDAC models with constitutive NFATc1 expression or also functions similarly in other PDAC models, we used KPC PDAC cells. KPC cells are derived from the $K_{\text {ras }}{ }^{G 12 D} ; T_{P 53}{ }^{R 172 H /+}$ (KPC) mice. We subjected KPC cells to CRISPR/Cas9mediated knockout of Ezh2 and analyzed Gata6 expression in these cells. There was an upregulation of Gata6 mRNA and protein expression upon EZH2 deletion (Figure $27 \mathrm{I} \& \mathrm{~J}$ ), thereby confirming that $\mathrm{EZH} 2$-dependent repression of Gata6 transcription is also seen in murine PDAC cells with endogenous NFATc1 expression. 

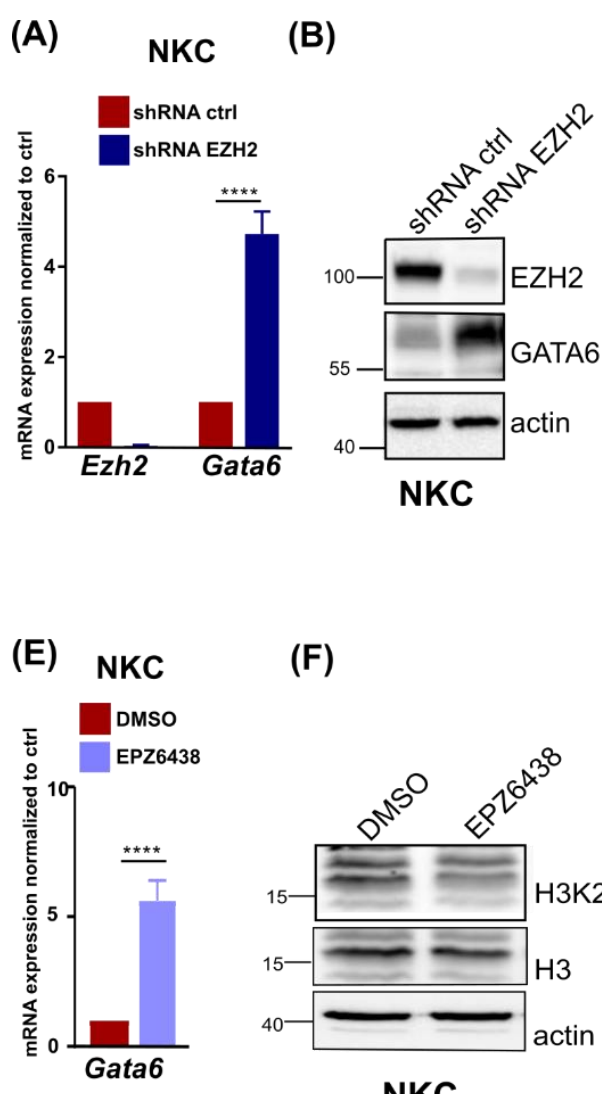

NKC
(C)

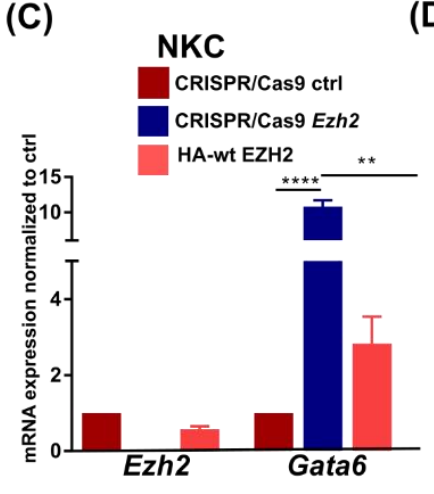

(D)

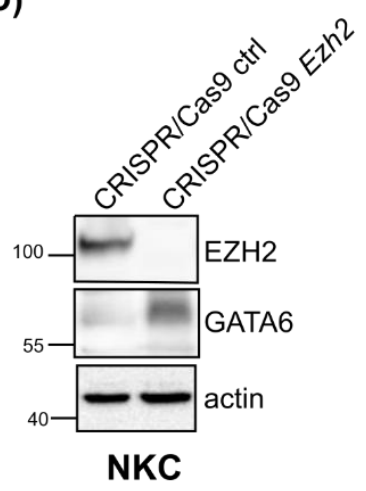

(G)

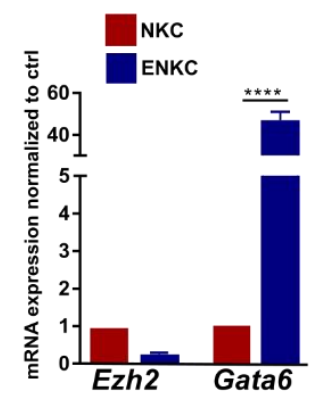

(H)

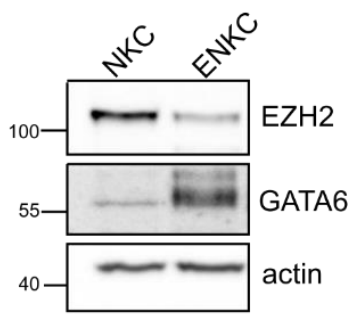

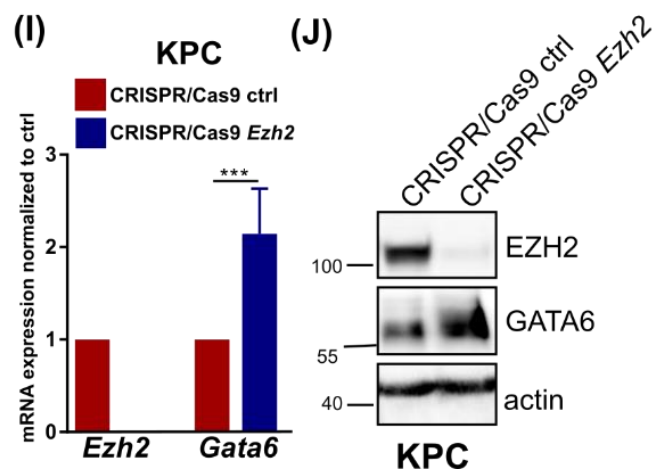

Figure 27: EZH2 deficiency leads to increased GATA6 expression in murine PDAC cells

(A) qPCR and (B) western blot analyses depicting increased GATA6 expression in NKC cells upon shRNA-mediated EZH2 knockdown. (C) qPCR showing decrease in Gata6 expression upon re-expression of EZH2 in CRISPR/Cas9-mediated EZH2 knockout NKC cells. (D) Western blot analyses depicting increased GATA6 expression in NKC cells upon CRISPR/Cas9 mediated EZH2 knockout cells. (E) qPCR illustrating reduction in Gata6 expression upon treatment of NKC cells with EZH2 inhibitor Tazemetostat (EPZ6438). (F) Expression analysis of H3K27me3 levels to confirm successful treatment with Tazemetostat (EPZ6438) (500 nM for 72 hours). H3 is the loading control. (G) qPCR and $(\mathbf{H})$ western Blot analyses depicting increased GATA6 expression in primary PDAC cells derived from $E Z H 2^{f l l} ;$;caNFATc1;Kras ${ }^{G 12 D}$ (ENKC) in comparison to PDAC cells derived from caNFATc1;Kras ${ }^{G 12 D}$ (NKC) mice. (I) qPCR and (J) Western Blot analyses depicting increased GATA6 expression in KPC cells upon CRISPR/Cas9 mediated EZH2 knockout. Two-way ANOVA was used to check the significance of the $q P C R$ results. $p<0.05$ was considered significant. 


\section{RESULTS}

\subsubsection{EZH2 represses GATA6 expression in human PDAC}

Our next question was to understand whether EZH2-dependent GATA6 repression also occurs in human PDAC. First, we took advantage of publicly available EZH2 ChIP-seq data accomplished in PANC-1 (Tzatsos et al. 2013). PANC-1 is a human pancreatic cancer epithelial cell line which is categorized as a basal-like subtype PDAC cell line (Diaferia et al. 2016). The ChIP-seq raw data was retrieved and analyzed in usegalaxy server using the default parameters. The EZH2 enrichment profile was visualized on IGV which revealed a significant enrichment of EZH2 on the GATA6 TSS/promoter (Figure 28A), confirming that EZH2 binds on the GATA6 gene in human PDAC as well. To validate whether $\mathrm{EZH} 2$ also blocks GATA6 transcription, we subjected PANC-1 cells to CRISPR/Cas9 mediated EZH2 deletion and tested for GATA6 expression in CRISPR/Cas9 control vs CRISPR/Cas9 EZH2 KO PANC-1 cells. As predicted, EZH2 deletion significantly increased GATA6 mRNA and protein expression (Figure 28B\&C), yet again supporting that the EZH2-GATA6 axis also persists in human PDAC.

(A)

PANC-1

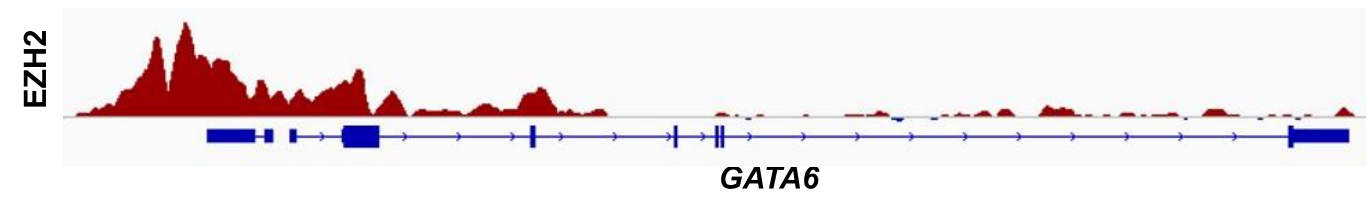

(B)

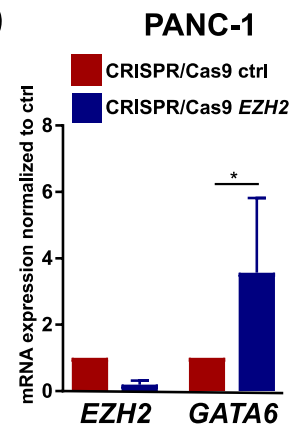

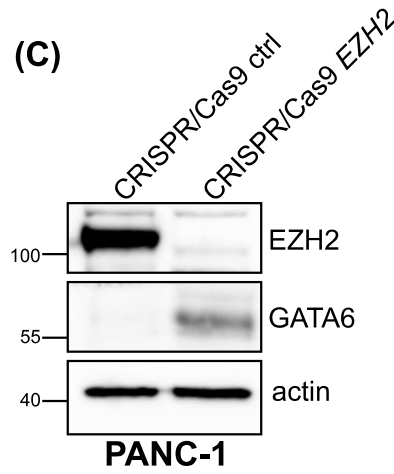

(C)

PANC-1

Figure 28: EZH2 regulates GATA6 expression in human PDAC cell line PANC-1.

(A) IGV profile demonstrating EZH2 occupancy on the GATA6 TSS in PANC1 cells as defined by ChIP-seq analysis (Tzatsos et al. 2013). (B) qPCR and (C) western blot analyses depicting increased GATA6 expression in PANC-1 cells upon CRISPR/Cas9mediated EZH2 knockout. Two-way ANOVA was used to check the significance of the qPCR results. 
We further aimed to validate the EZH2-GATA6-axis in Patient-derived xenograft (PDX) models. PDX models are currently largely used as preclinical model systems (Li 2015; C. Xu et al. 2019). We took advantage of PDAC PDX models established in our department which have been utilized to generate primary PDX derived PDAC cells. We utilized cells (GCDX5) generated from one of the PDX tumors (PDX5) to explore EZH2-mediated Gata6 repression. When we exposed these cells to siRNA-mediated EZH2 knockdown, we observed a significant upregulation of GATA6 mRNA and protein. Moreover, the pharmacological blockade of EZH2 activity by treating GCDX5 cells with EZH2 inhibitor also increased GATA6 transcription. These results further strengthen the finding that EZH2 dependent GATA6 regulation persists in human PDAC.

(A)

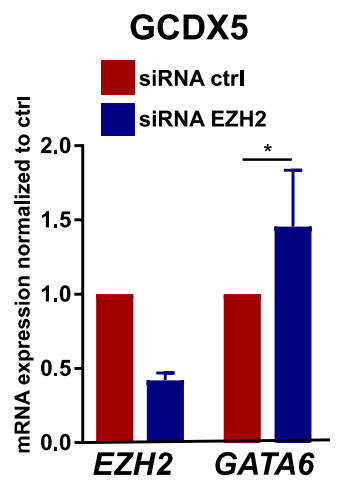

(C)

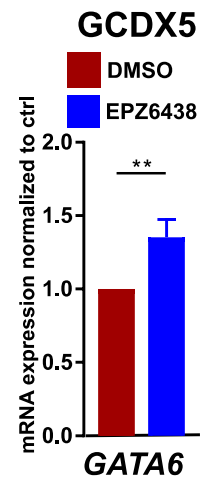

(B)

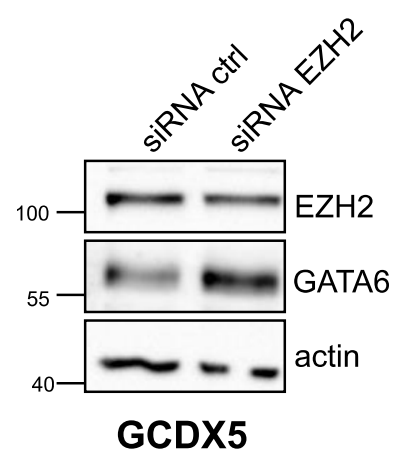

(D)

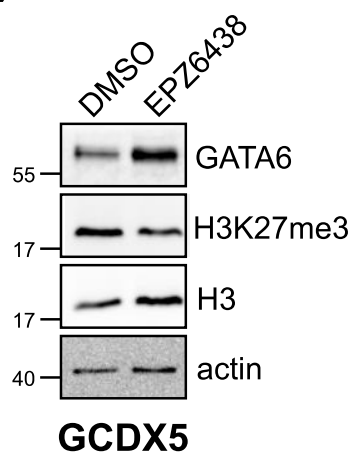

Figure 29: EZH2 regulates GAT6A expression in cells derived from PDX models.

(A) qPCR and (B) western blot analyses depicting increased GATA6 expression in GCDX5 cells upon siRNA-mediated knockdown of EZH2. (C) GPCR and (D) western Blot analyses depicting increased GATA6 expression in GCDX5 cells upon and Tazemetostat (EPZ6438) treatment ( $1 \mu \mathrm{M}$ for 72 hours). Two-way ANOVA was used to check the significance of the GPCR results. 


\section{RESULTS}

\subsubsection{The EZH2-GATA6 axis exists in human PDAC specimens}

To further investigate the significance of the EZH2-GATA6 axis in human PDAC patient specimens, we performed immunohistochemical staining analyses of GATA6 in the PDAC TMA mentioned in Figure 5. We applied the immunoreactive score (IRS) to quantify GATA6 expression in each sample. Tissues with IRS $\leq 3$ were considered as tissues with low GATA6 levels and tissues with IRS > 3 were considered as high GATA6 expressing tissues. IRS of GATA6 was further correlated with the IRS of EZH2. Remarkably, GATA6 expression was reduced in a significant number of EZH2-high tumors (Figure 30), indicating that EZH2-dependent GATA6 repression is evident in a subgroup of human PDAC specimens. Taken together, these data suggest that EZH2-GATA6 axis also exists in human PDAC.

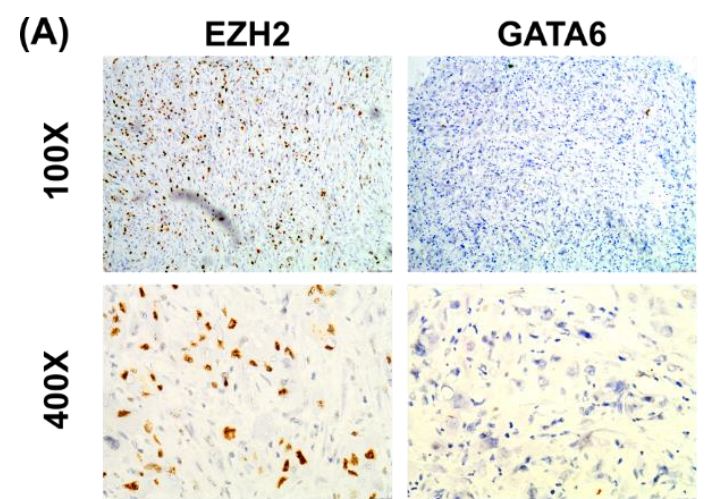

Patient 1

\section{EZH2}

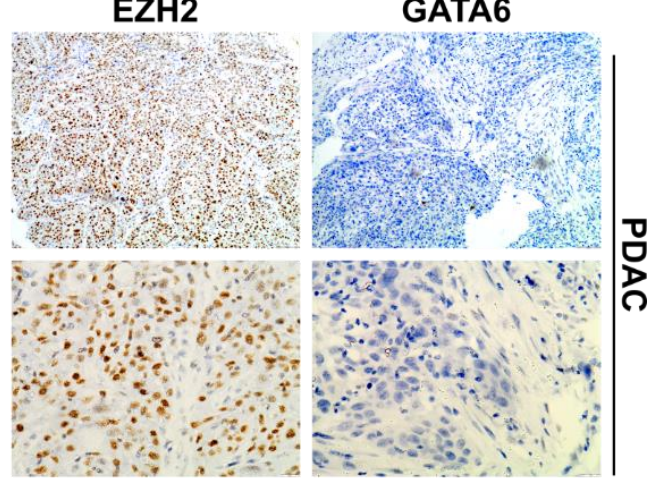

Patient 2

(B)

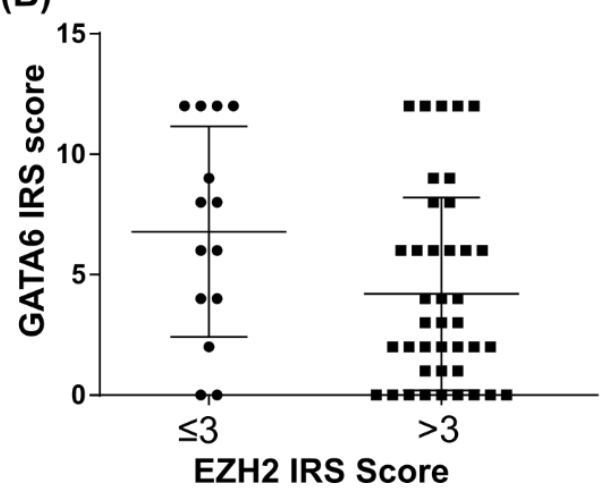

Figure 30: EZH2 dependent GATA6 regulation is evident in the subpopulation of PDAC patients.

(A) Representative images from two PDAC patients showing EZH2 and GATA6 expression in human PDAC tissue. Magnification -100X and 400X; Scale bar - $100 \mu \mathrm{m}$ and $10 \mu \mathrm{m}$. (B) Graph depicting GATA6 expression in patients expressing low (IRS $\leq$ 3 ) and high (IRS > 3) EZH2 expression. Each dot represents a patient from the TMA described in Figure 5 . Values represent mean \pm SD. Significance was determined by two-tailed unpaired student's $t$-test. 


\subsubsection{GATA6 targets are enriched upon EZH2 depletion}

To explore whether EZH2 also interferes with transcription programs regulated by GATA6, we utilized publicly available GATA6 ChIP- and RNA- seq data in PDAC (Martinelli et al. 2016). From the GATA6 ChIP-seq data, we set a threshold of FDR $<1$ to the output from Diffbind and analyzed those GATA6 binding regions using the GREAT tool to obtain the genes associated with these regions. We thereby identified 7065 GATA6 target genes. Subsequently, we intersected those direct GATA6 target genes with genes for which RNA-seq analysis revealed a significant $(q \leq 0.05)$ downregulation upon knockout of Gata6 (172 genes). Hence, we discovered 58 significant genes that are boundand activated by GATA6 (Figure 31A). Subsequently, we performed GSEA with these GATA6 targets on our transcriptome data. Interestingly, GATA6 targets were positively enriched upon EZH2 depletion (Figure 31B), suggesting that EZH2 controls GATA6-dependent transcriptional programs in PDAC.

(A)

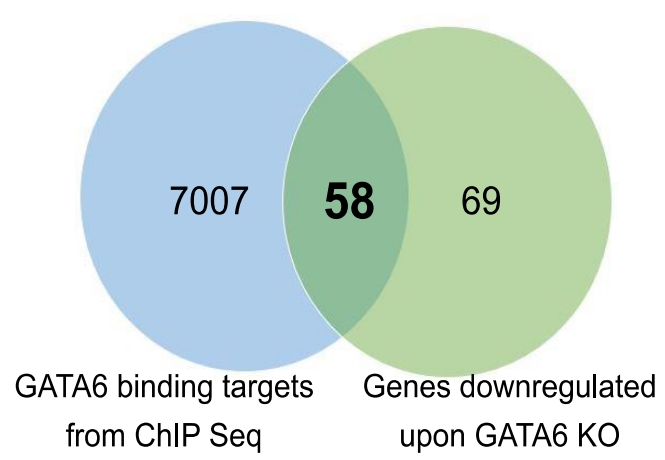

(B)

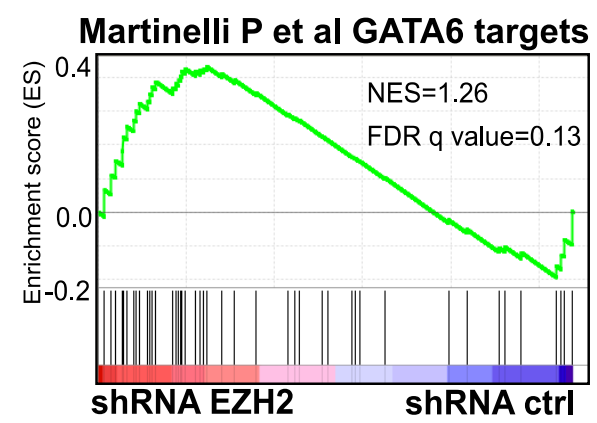

Figure 31: Direct downstream targets of GATA6 are enriched upon EZH2 depletion.

(A) Venn diagram showing direct Gata6 target genes which are transcriptionally activated by the transcription factor as identified by Martinelli et al (Martinelli et al. 2016). (B) GSEA plot showing enrichment of Gata6 targets identified in A upon EZH2 depletion. Significance was determined based on NES and FDR q value. 


\section{RESULTS}

\subsubsection{Gata6 knockdown can rescue the effects of EZH2 depletion on invasion}

In order to explore the functional significance of GATA6 in curbing EZH2 mediated PDAC progression, we depleted Gata6 in shRNA EZH2 NKC cells using siRNA strategies. Figure 32A depicts the confirmation of knockdown of Gata6. Subsequently, we performed an invasion assay to investigate whether Gata6 knockdown can rescue the invasive capacity of EZH2-deficient PDAC cells. The number of invading cells increased significantly upon Gata6 knockdown in EZH2-depleted cells (Figure 32B), arguing that Gata6 depletion partially restores the invasive potential of shRNA EZH2 NKC cells. These results suggest that Gata6 repression is essential for EZH2-driven PDAC cells invasion.

To further understand the role of GATA6 in impeding the EZH2-dependent repression of gene signatures associated with the classical PDAC subtype, we examined the expression of few classical genes in the presence and absence of EZH2 and upon GATA6 depletion. The classical genes were selected from the previously described Pure-classical gene set defined by Puleo and colleagues (Puleo et al. 2018). Consistent with the observed enrichment of pure classical and basal-like gene signatures in shRNA EZH2 and shRNA control NKC cells, respectively, pure classical genes were upregulated upon sole EZH2 depletion. However, GATA6 depletion partially rescued increased classical target gene expression observed upon EZH2 deficiency (Figure 32C) suggesting that GATA6 is required for $\mathrm{EZH} 2$ mediated abrogation of classical gene expression signatures.

Taken together, these data demonstrate that GATA6 expression is essential for the abrogation of invasive potential of features of PDAC and acquisition of a classical subtype state upon EZH2 depletion. 


\section{RESULTS}

(A)

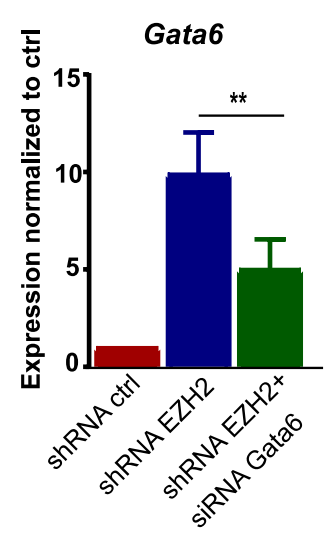

(B)

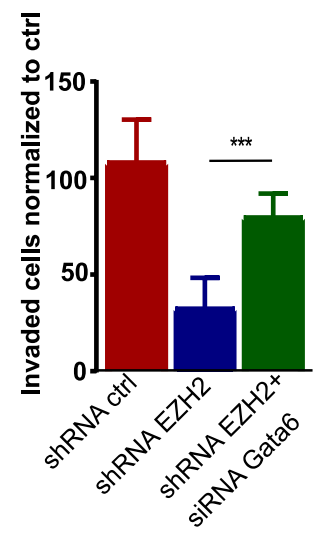

(C)

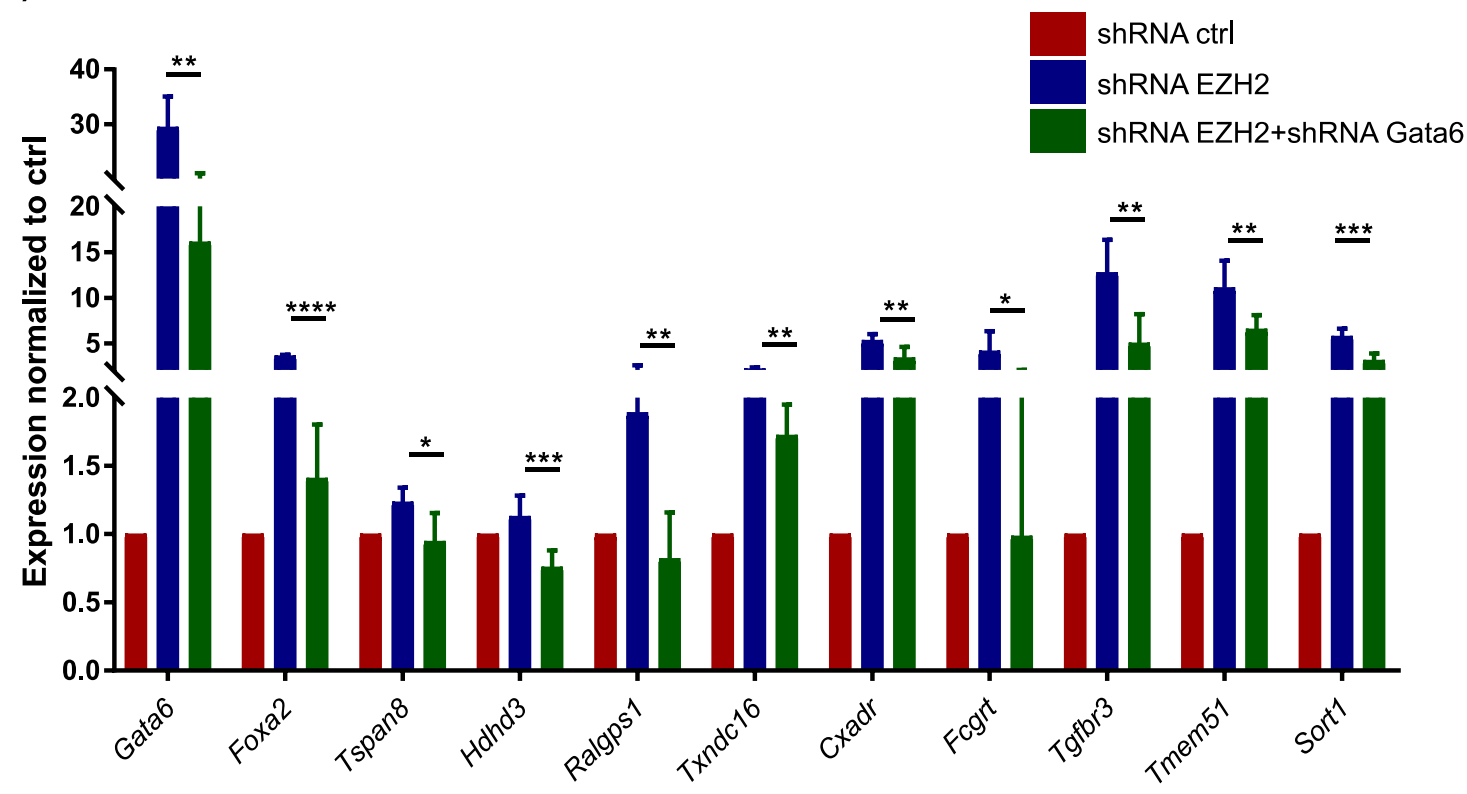

Figure 32: GATA6 knockdown partially rescues the invasion effects mediated by EZH2 deficiency.

(A) qPCR demonstrating successful knockdown of Gata6 in shRNA EZH2 NKC cells.

(B) Boyden chamber assay to determine NKC cell invasion in the presence and absence of EZH2 and upon Gata6 depletion. (C) qPCR performed in NKC cells to evaluate a selection of target genes associated with the pure classical PDAC subtypes as defined by Puleo et al (Puleo et al. 2018). Two-tailed unpaired student's t-test was used to test the statistical significance.

In summary, our findings link transcriptional GATA6 repression to EZH2dependent tumor progression in PDAC and suggest that pharmacological interference with EZH2 activity in a subset of PDAC patients can re-install GATA6 expression in favor of a less aggressive tumor phenotype (Patil $S$ et al. in revision). 



\section{DISCUSSION}

\subsection{Polycomb group proteins in the regulation of differentiation processes}

Polycomb group ( $P c G$ ) proteins regulate several processes during development including cell fate determination, lineage specification, stem cell regulation and maintaining homeostasis (Bracken et al. 2006; Ringrose and Paro 2004; Schuettengruber and Cavalli 2009; Surface, Thornton, and Boyer 2010). In the course of embryonic development, they control transcriptional programs by restricting the expression of specific genes. They particularly repress genes linked to differentiation and maintain the repressed state of the genes in an adult differentiated cell (Sparmann and Van Lohuizen 2006). Along with their critical role in development and differentiation, several studies show a significant connection between polycomb proteins and cancer (Hormaeche and Licht 2007; Richly, Aloia, and Di Croce 2011; Sauvageau and Sauvageau 2010; Valk-Lingbeek, Bruggeman, and Van Lohuizen 2004). PcG proteins carry out their functions by forming multiprotein complexes like PRC1 and PRC2. $\mathrm{EZH} 2$ is one of the most important members of the PRC2 complex and is found to be vital in ensuring proper development of various organs including the pancreas (Chou et al. 2015; San et al. 2016). This is very well reflected in our study where we see that pancreas specific homozygous EZH2 knockout mice are lethal and show various developmental abnormalities of the pancreas.

\subsection{EZH2 in pancreas development and cancer progression}

Since the past few years, EZH2 has gained a lot of attention regarding its deregulation in various cancer entities. For instance, EZH2 overexpression is seen in breast and prostate cancer, linking its abundant expression to poor prognosis and advanced tumor stages (Bachmann et al. 2006; Kleer et al. 2003). Similarly, in pancreatic cancer, consistent with previous studies (N. M. Chen et al. 2017; Ougolkov, Bilim, and Billadeau 2008), our findings also show increased EZH2 levels in dedifferentiated high-grade human PDAC.

EZH2 overexpression in cancer has been associated with increased proliferation (Ougolkov, Bilim, and Billadeau 2008), stemness (Y. H. Chen, Hung, and Li 2012), migration (Smits et al. 2010), invasion (Han et al. 2016) 


\section{DISCUSSION}

and metastasis (Rao et al. 2010; Richter et al. 2009). In line with these reports, we made similar observations upon performing functional assays in NKC PDAC cells in vitro. Upon EZH2 depletion, cells exhibited reduced proliferation, anchorage-independent growth and invasive capabilities confirming that $\mathrm{EZH} 2$ deficient cells reduce their tumor-promoting activities. One of the reasons for the failure to sufficiently eradicate cancer cells has been credited to the stemness property of cancer cells which lead to therapy resistance and tumor relapse (Nangia-Makker, Hogan, and Raz 2018; Shlush et al. 2017). Our sphere-formation assay in NKC PDAC cells depicts that upon loss of EZH2, NKC cells lose their stemness property. Accordingly, the RNA-seq analysis suggests that EZH2 regulates transcriptional programs that mediate stemness in PDAC cells. These findings argue that EZH2 depletion can create a shift towards reduced stemness features which might reduce the likelihood of PDAC relapses.

We utilized the caNfatc1; Kras ${ }^{G 12 D}$ transgenic mouse models with wildtype Ezh2 expression or upon heterozygous Ezh2 expression to investigate the implications of $\mathrm{EZH} 2$ depletion in PDAC in vivo. Although homozygous mice would aid better in characterizing the role of $\mathrm{EZH} 2$, the heterozygous mice already displayed differences regarding changes in cellular differentiation status and metastasis. Despite the EZH2-status dependent differences in tumor biology in these models, we did not observe significant differences between the survival of caNfatc1;Kras ${ }^{G 12 D}$ and $E Z H 2^{f / /+}$ caNfatc1;Kras ${ }^{G 12 D}$ mice. Given that $\mathrm{EZH} 2$ is critical for proper development of the pancreas and based on our observation that $\mathrm{EZH} 2$ heterozygous mice of advanced age displayed initial signs of pancreatic insufficiency and atrophy, the comparable median survival between the groups can be best explained by developmental issues. We speculate that the utilization of an inducible mouse model (Guerra and Barbacid 2013; Martinelli et al. 2016) which facilitates EZH2 depletion after the completion of pancreatic development would favor a better judgment on the impact of EZH2 activity on tumor progression and mice survival. However, our GEMM harboring EZH2 heterozygous deficiency already displays reduced tumor incidence and metastasis. Hence, despite the limitations of our PDAC model, it reflects the human situation to a significant extent. 
caNfatc1;Kras ${ }^{G 12 D}$ mouse model harbor the oncogenic Kras ${ }^{G 12 D}$ mutation and constitutive expression of the inflammatory transcription factor NFATc1. Considering the implication of inflammation in boosting PDAC development (Baer et al. 2014; Yadav and Lowenfels 2013; Ye et al. 2019; Young et al. 2019), this well-characterized transgenic mouse serves as a good model system to study PDAC carcinogenesis. Additionally, these mice undergo all steps of pancreatic carcinogenesis, thus highly mimicking human PDAC development (Baumgart et al. 2014). Besides, Cre-mediated expression of HAtagged NFATc1 in this model occurs constitutively under the control of the Rosa26 promoter, thus prohibiting the previously described alterations (N. M. Chen et al. 2017) of NFATc1 expression upon interference with EZH2 activity. Furthermore, EZH2 has been identified as a transcriptional NFATc1 target gene (Baumgart et al. 2014) which means NKC mouse model expresses high levels of $\mathrm{EZH} 2$, thus characterizing this model as a practicable tool to study the tumor biological consequences and the mechanistic basis of EZH2 overexpression in PDAC. However, in the context of constitutive NFATc1 activation, homozygous Ezh2 depleted mice are lethal which hinders us from examining homozygous EZH2 loss in these mice. Interestingly, in another GEMM which is also based on Kras ${ }^{G 12 D}$ but shows endogenous NFATc1 expression (EZH2 $2^{f / f t} ; K r a s^{G 12 D}$ (KEC)), this was not to be observed and the mice were viable even upon homozygous Ezh2 depletion (N. M. Chen et al. 2017; Mallen-St. Clair et al. 2012). Hence, constitutive NFATc1 activation seems to potentiate the functional implications of Ezh2 deficiency in PDAC development. However, the EZH2GATA6 axis was also confirmed in systems with endogenous NFATc1 expression and in the context of additional genetic drivers of PDAC (e.g. gain of function mutations of TP53).

Although the functional implications of EZH2 in cancer are published in various reports (Y. H. Chen, Hung, and Li 2012; Han et al. 2016; Ougolkov, Bilim, and Billadeau 2008; Rao et al. 2010; Richter et al. 2009; Smits et al. 2010), very few studies exist that decode the specific function of this histone methyltransferase in PDAC. Consistently, very little is known about the mechanistic functions and direct targets of EZH2, especially in pancreatic cancer. Our study describes ChIP- and RNA-seq analysis which provides insights into the mechanistic background of EZH2-dependent gene regulation in PDAC and links EZH2 
activity to suppression of transcriptional programs fostering differentiation and a better outcome of PDAC patients.

\subsection{Role of epigenetic modulations in mediating cancer cell plasticity}

Cancer cells have a high level of cellular and molecular plasticity which is predominantly regulated at the level of gene transcription (Flavahan, Gaskell, and Bernstein 2017). Their ability to interconvert between different states allows them to grow and spread across different sites (Roe et al. 2017). EMT and stemness represent crucial factors for cancer cell plasticity which have a major impact on metastasis and therapeutic resistance, respectively (Polyak and Weinberg 2009). Many epigenetic modulators regulate transcription programs that drive this transition. For instance, epigenetic alterations initiated by transcription factors like SNAI and ZEB1 modulate the chromatin, consequently controlling the transcription of genes involved in EMT (Nieto et al. 2016). In a study in small cell lung cancer (SCLC), amplification of the transcription factor NFIB offered genome-wide chromatin accessibility subsequently promoting gene expression controlling metastasis programs (Denny et al. 2016). Recent evidence suggests that reprogramming of the enhancer landscape mediates metastatic potential in pancreatic cancer cells (Roe et al. 2017). Few studies indicate the role of $\mathrm{EZH} 2$ in interacting with EMT associated factors. For instance, EZH2 promotes Snail-mediated recruitment of RING1A/B to the CDH1 promoter (encoding for E-Cadherin) to repress its expression, thereby promoting cell migration in PANC-1 cells (Chen et al. 2014). Furthermore, few studies also suggest that $\mathrm{EZH} 2$ binds directly to the $\mathrm{CDH} 1$ promoter to repress its expression, thus promoting cell migration and invasion (Han et al. 2016; Qazi et al. 2012). However, in our study, we do not observe any major differences in the expression of EMT-related gene signatures upon loss of $E Z H 2$. Interestingly we still discovered a potential link between EZH2 expression and metastasis in vivo. However, there are a few reports suggesting EMT-independent mechanisms driving PDAC metastasis in GEMMs. For instance, Zheng et al demonstrate that loss of Twist1 and Snai1 does not influence metastases frequency (Zheng et al. 2015) and further fluorescencebased lineage tracing experiments confirmed non-EMT associated metastasis in mouse models of PDAC (Y. Chen et al. 2018). Considering these studies 
arguing that the EMT process is dispensable for metastasis in PDAC, we contemplate a possibility that metastasis could have been influenced by processes other than EMT in our mouse models. It is also possible that the implication of EZH2 on EMT was prominent in mice but not as well captured in our in vitro systems. To better understand the implication of EZH2 on EMT in vivo, the tumors from the wildtype and EZH2 depleted mice could be stained with antibodies recognizing EMT markers.

There are few studies in breast cancer investigating the differences in epigenomic patterns between primary and metastatic sites. Priedigkeit and colleagues showed in their study that $20 \%$ of patients with ERBB2-HER2 negative tumors at the primary tumor site showed ERBB2/HER2 positive brain metastasis (Priedigkeit et al. 2017). In a recent report by Cai et al., they studied the transcriptional differences between cells from tumors in primary and metastasized sites (brain and lung) of breast cancer utilizing high throughput sequencing approaches (ChIP-seq, RNA-seq, ATAC-seq, and HiChIP). They claim that very few of those differences are driven by genetic mutations, while the majority of these are promoted by epigenetic alterations indicating the relevance of epigenomic reprogramming in fostering cellular plasticity of cancer (Cai et al. 2020). Similarly, pancreatic cancer, which is a highly heterogeneous tumor, also displays a high degree of cellular plasticity which is largely mediated by differences in epigenetic landscapes. Lomberk et al. performed a comprehensive analysis of epigenomic landscapes underlying PDAC subtypes in patient-derived tumor xenografts (PDTXs) combining ChIP-seq for various histone marks, RNA-seq and DNA methylation approaches where they associate the epigenomic pattern with PDAC heterogeneity. Moreover, they identify MET as a crucial transcription factor expressed in basal-like PDAC subtypes and also show that siRNA mediated depletion of MET in PDTXderived cell lines mediates a shift from basal to classical PDAC subtype, highlighting the influence of epigenetics in PDAC plasticity (G. Lomberk et al. 2018). Considering the implications of $\mathrm{EZH} 2$ in promoting metastasis and stemness in our studies, manipulating EZH2 expression to tackle PDAC plasticity could be considered as a therapeutic strategy. 


\section{DISCUSSION}

\subsection{EZH2 in PDAC subtype identity}

In the last decade, various molecular subtypes of PDAC have been described. Although just a few genes overlap between different classification systems and despite discrepancies in the definition of molecular PDAC subtypes, two subtype classes were consistently represented throughout these studies and were found to have prognostic relevance - basal-like (also called squamous or quasi-mesenchymal (QM)) and classical subtypes (P. Bailey et al. 2016; Collisson et al. 2011; Moffitt et al. 2015; Puleo et al. 2018). While the basal subtype is correlated with a higher tumor grade and poor survival, the classical subtype is relatively well-differentiated and exhibits a better prognosis ( $P$. Bailey et al. 2016; Collisson et al. 2011).

Poorly differentiated

Low survival

\section{Well differentiated}

High survival

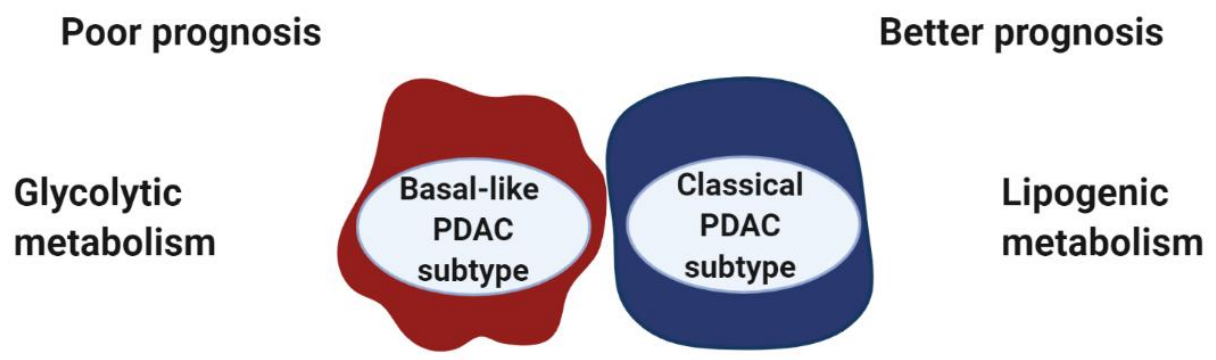

\begin{abstract}
Increased expression of mesenchymal genes
\end{abstract}

\author{
Increased expression of \\ epithelial genes
} Chemotherapy: Worse
response
Chemotherapy: Better response

Figure 33: Schematic depicting two main PDAC subtypes and its features.

Two PDAC subtypes represented in all subtyping studies combined are basal-like and classical PDACs. There is a clear distinction between the features of these two subtypes regarding differentiation status, survival, prognosis, metabolism, gene signatures and response to chemotherapy. 
Molecularly, basal tumors show increased TP63 expression (Mueller et al. 2018) and express genes related to EMT (Martens et al. 2019; Nicolle et al. 2017). In contrast, classical tumors are characterized by expression of pancreatic developmental transcription factors and epithelial genes (P. Bailey et al. 2016). Dissimilar with classical subtypes, basal subtypes lose expression of master endodermal transcription factors that are required to maintain endodermal differentiation (Adamo et al. 2017). Additionally, basal subtypes regularly carry mutations in genes encoding chromatin regulatory proteins like KDM6A (Andricovich et al. 2018). Reports also suggest a correlation of PDAC subtypes with metabolism. While basal tumors are enriched for genes regulating glycolytic pathways, classical tumors show enrichment for lipogenic pathways (Daemen et al. 2015; Martens et al. 2019; Nicolle et al. 2017). Evidence also suggests that these subtypes differ in their responses to chemotherapy where basal tumors show an overall worse chemotherapeutic response compared to classical tumors (Aung et al. 2019; P. Bailey et al. 2016; Collisson et al. 2011; O'Kane et al. 2020). Together, there exists evidence for a classical/basal-like subtype system in PDAC which embodies prognostic and predictive relevance. Hence, molecular-subtype-driven stratification in PDAC treatment might represent a promising strategy to tackle PDAC.

Differential gene expression analysis performed in our study revealed that EZH2 loss led to the enrichment of transcriptional programs fostering a favorable prognosis and promoting differentiation as well as a classical PDAC subtype state. Additionally, basal-like gene signatures were negatively enriched upon EZH2 depletion. These observations point out to a possibility of the existence of the EZH2-high subgroup in the basal-like PDAC subtype. However, we did not observe differences in EZH2 expression per se between classical and basal-like PDAC subtype states in the published datasets (P. Bailey et al. 2016; Diaferia et al. 2016; Puleo et al. 2018). This confirms that the expression of $\mathrm{EZH} 2$ alone cannot be used as a predictive marker to determine the subtype. To understand if manipulating EZH2 truly converts PDAC tumors from basal to classical PDA tumors, EZH2 could be depleted in basal tumors and further the $\mathrm{EZH} 2$ proficient and deficient basal tumors can be analyzed for classical/basal PDAC features by staining for classical markers (E-Cadherin, GATA6, HNF1A) and basal markers (KRT5, KRT14, TP63). However, we 


\section{DISCUSSION}

speculate that EZH2 functions by repressing the classical genes rather than modulating the basal genes.

\subsection{GATA6 as a marker of the classical PDAC subtype}

The transcription factor that was consistently mentioned in all PDAC molecular subtyping studies was GATA6. The classical PDAC subtype defined by both Moffit and Collison expressed elevated levels of GATA6 (Collisson et al. 2011; Moffitt et al. 2015). Furthermore, Bailey et al. reported that GATA6 was epigenetically repressed in the squamous subtype (P. Bailey et al. 2016). The aforementioned subtyping studies and various other reports (G. Lomberk et al. 2018; Puleo et al. 2018; Seino et al. 2018; The Cancer Genome Atlas Research Network and Raphael 2017) characterize the expression and activity of GATA6 as the crucial driver of PDAC subtype identity.

GATA6 belongs to the family of GATA transcription factors that contains two highly conserved zinc-finger DNA-binding domains that recognize an $(\mathrm{A} / \mathrm{T}) \mathrm{GATA}(\mathrm{A} / \mathrm{G})$ consensus nucleotide sequence. There are six GATA members: while GATA 1-3 are predominantly expressed in the hematopoietic lineage, GATA 4-6 are mainly present in the endodermal lineage (RodríguezGarcía, Sola-Landa, and Barreiro 2017; Tiyaboonchai et al. 2017). GATA6 drives endodermal lineage differentiation by transcriptionally activating lineage defining transcription factors (Fisher et al. 2017). Consistently, Gata6 maintains epithelial differentiation and suppresses mutant Kras-driven tumorigenesis in the mouse pancreas by activating differentiation-related transcription programs (Martinelli et al. 2016).

In pancreatic cancer models, GATA6 blocks dedifferentiation and abrogates EMT programs. Moreover, dedifferentiated basal-like PDAC tumors with low GATA6 expression associate with poor prognosis and low response to 5fluorouracil (5-FU) based adjuvant therapy (Martinelli et al. 2017). In addition to these studies, results from the COMPASS trial identified GATA6 expression as a robust surrogate biomarker for differentiating classical and basal PDAC subtypes utilizing RNA sequencing and RNA in situ hybridization (ISH) techniques (Aung et al. 2019). Besides, in the same COMPASS trial samples, Kane et al. validated GATA6 as the prime classifier of the classical PDAC 
subtype, while its expression is low or almost absent in basal-like tumors (O'Kane et al. 2020).

Utilizing high throughput sequencing approaches, our findings identified Gata6 as a key target of EZH2-dependent transcriptional repression in PDAC. Notably, GATA6 depletion in EZH2 deficient cells re-installed the invasive potential of PDAC cells and also decreased the expression of classical PDAC subtype genes, implying that EZH2-mediated GATA6 repression is crucial for the tumor-promoting activities of the histone methyltransferase. However, to what extend GATA6 expression impacts on EZH2-driven PDAC biology remains to be elucidated, for instance by utilizing in vivo models with and without Gata6 expression in the absence of EZH2.

\subsection{PDAC subtype switching}

PDAC subtypes are controlled at the level of transcription (P. Bailey et al. 2016; Collisson et al. 2011; Moffitt et al. 2015; Puleo et al. 2018). Considering that PDAC exhibits a high degree of plasticity and given that epigenetic alterations are reversible, PDAC subtypes are potentially interconvertible. Importantly, classical PDAC tumors exhibit a better prognosis and higher chemotherapeutic susceptibility, thus underinning that subtype switching towards the classical PDAC subtype constitutes an attractive strategy in PDAC treatment. Subtype switching has for instance been reported for basal-like PDAC subtypes harboring mutations in the histone demethylase encoding gene KDM6A. Loss of KDM6A fosters aberrant rewiring of super-enhancers regulating oncogenes such as $\triangle N p 63, R U N X$, and MYC, thereby promoting dedifferentiation to the squamous-like subtype and metastatic PDAC. Furthermore, the study confirms in vivo that KDM6A-deficient PDAC is more susceptible to BET inhibitors which reverse basal differentiation and restrain PDAC growth. Overall, this study reveals reprogramming of super-enhancers as a crucial mechanism for subtype switching in pancreatic cancer (Andricovich et al. 2018). Besides the chromatin regulatory proteins that control subtype-defining transcription programs, subtype switching is also controlled by pioneer transcription factors. For instance, Adams and colleagues provide detailed evidence stating Gliomaassociated oncogene homology 2 (GLI2) transcription factor as the critical regulator of PDAC subtype switching. GLI2 mediates hedgehog signaling and 


\section{DISCUSSION}

was found to be highly expressed in basal-like pancreatic cancer cell lines and patient tissues. GLI2 activation could sufficiently switch PDAC cells from classical to basal-like subtype harboring enhanced EMT potential. Additionally, depletion of this transcription factor could suppress basal transcription programs, suggesting interfering with GLI2 activity might provide a promising strategy to push PDAC cells towards a less aggressive, classical subtype (Adams et al. 2019). These studies emphasize the significant degree of plasticity modulated by the chromatin factors and transcription factors in PDAC subtypes.

\subsubsection{Strategies to induce GATA6 expression for subtype switching}

Switching from one subtype to another involves transcriptional reprogramming of endodermal developmental and differentiation programs. Considering the involvement of GATA6 in PDAC development, progression and differentiation (Hermann et al. 2014; Martinelli et al. 2016, 2017) and its implications in the classical PDAC subtype (Adams et al. 2019; Aung et al. 2019; P. Bailey et al. 2016; Collisson et al. 2011; Moffitt et al. 2015; O’Kane et al. 2020; Puleo et al. 2018), upregulation of GATA6 expression is an attractive strategy to promote and maintain classical subtype identity. Consistently, the induction of GATA6 was observed in tumors upon switching from basal to classical subtypes in lung and skin cancers (Biehs et al. 2018). Furthermore, the downregulation of GATA6 mediated commitment to squamous subtype in lung cancer models (Cheung et al. 2013). Consistently, similar observations are made in PDAC subtype inter-conversion. For instance, while GATA6 expression declined in GLI2-induced basal PDAC cells, loss of GLI2 resulted in increased GATA6 expression and acquisition of classical gene signatures (Adams et al. 2019). Similarly, genetic depletion of the hepatocyte growth factor receptor MET in basal PDAC subtypes resulted in a shift towards the classical phenotype which involved upregulation of GATA6-dependent transcription programs (G. Lomberk et al. 2018). These studies emphasize the role of the transcriptional regulator GATA6 in subtype switching in pancreatic cancer. However, the strategies to re-induce GATA6 expression are not well understood.

First insights on the dynamic regulation of GATA6 expression came from reports focusing on developmental processes. These studies suggest that the 98 
regulation of GATA6 expression occurs at the chromatin level (Fisher et al. 2017; Smith, Singh, and Dalton 2010; Suzuki et al. 2015), for instance by transcriptional repression by PcG proteins. Gata6 expression is found to be tightly regulated by polycomb proteins during cardiac development (Kang et al. 2010). Furthermore, genome-wide shRNA screening in embryonic stem cells identified BCOR, an associated member of the PRC1 complex member RING1B as an essential repressor of the GATA6 gene (Cooper and Brockdorff 2013). Further emphasizing the impact of the PcG family of chromatin regulatory proteins in GATA6 regulation, the PRC2 core member EED maintains neural stem cell differentiation by repressing GATA6 transcription (Sun et al. 2018). Finally, loss of the histone methyltransferase EZH2 in mouse embryos resulted in the differentiation of germ layers due to the de-repression of Hoxb1, Hand1, and Gata6 (Huang et al, 2014).

However, the mechanisms controlling the expression of GATA6 in cancer remain mainly elusive. Our study explored EZH2 dependent Gata6 regulation in vitro in different PDAC murine (NKC, KPC) and human (PANC-1) cell lines upon transient knockdown and stable knockout of EZH2 as well as upon pharmacological EZH2 inhibition. Additionally, EZH2-dependent GATA6 regulation was recognized in PDX specimens at both $\mathrm{mRNA}$ and protein levels. Our analysis in human samples also revealed a subgroup of PDAC patients with EZH2 high/GATA6 low tumors, and these patients might be potentially exhibiting EZH2 dependent GATA6 regulation. Combining all the data, our findings identify a novel mechanism of GATA6 regulation in PDAC. EZH2 regulates Gata6 at the transcriptional level by binding on the TSS/promoter of the Gata6 gene and depositing the H3K27me3 repressive mark. Upon loss of $\mathrm{EZH} 2$, there is a reversal of this regulation which leads to increased Gata6 transcription and enrichment of GATA6 downstream targets. However, the mechanism behind the recruitment of EZH2 to the Gata6 locus is yet to be discovered. We have observed before that NFATc1 regulates EZH2 expression in PDAC (Baumgart et al. 2014) and biochemically interacts with the methyltransferase (unpublished data of the Hessmann group). Hence, NFATc1 might recruit EZH2 to the Gata6 promoter. Furthermore, one of the previous report suggest KDM2B-mediated EZH2 recruitment to the Gata6 locus (Tzatsos et al. 2013). Although the exact mechanism targeting EZH2 to the GATA6 gene 


\section{DISCUSSION}

remains to be elucidated, our data suggest EZH2 inhibition as a promising strategy to re-install GATA6 expression in a subgroup of PDAC.

\subsection{Pharmacological inhibition of EZH2 in PDAC}

$\mathrm{EZH} 2$ is a very attractive target in cancer therapy due to its oncogenic potential in various cancer types (Raman et al. 2005; Rao et al. 2010; Völkel et al. 2015; Zingg et al. 2015). Consequently, EZH2 inhibition was evaluated as a therapeutic strategy in many cancer entities (Huang et al. 2015a; McCabe et al. 2012; Miele et al. 2017; Tan et al. 2007). Besides, the data on the safety and efficacy of $\mathrm{EZH} 2$ inhibitors is already available from clinical trials in hematological malignancies and selected solid tumors (e.g. NCT04179864, NCT03010982). Consequently, pharmacological interference with EZH2 activity represents a therapeutic option with strong translational potential in PDAC. However, as described before in chapter 1.4.2, epigenetic mechanisms in general, and EZH2 activity in particular, underlay a strong context-dependency. Consequently, we proposed that the efficacy of EZH2 inhibition as a therapeutic strategy in PDAC might be restricted to a subgroup of PDAC which encompasses those molecular prerequisites required for beneficial EZH2 inhibition.

Given that our data suggest transcriptional repression of GATA6 as an essential mechanism underlying oncogenic EZH2 activity, we propose that the therapeutic efficacy of EZH2 inhibition is restricted to a subgroup of PDAC patients characterized by high EZH2 activity and low GATA6 expression. In this particular context, the EZH2 blockade might sufficiently re-install GATA6 expression with a subsequent shift towards gene signatures essential for maintaining classical subtype identity. Based on our analysis in human PDAC tissue the subgroup of EZH2high/GATA6low PDAC subtypes represents approximately $40 \%$ of PDAC patients. While pharmacological interference with the EZH2-GATA6 axis might be beneficial in this subset of PDAC, EZH2 inhibition might eventually prove less efficient in tumors which are either characterized by low EZH2 expression and activity and/or already express high levels of GATA6. Indeed, EZH2-independent mechanisms controlling GATA6 expression have been reported previously. For instance, some cancer entities including PDAC can be characterized by amplification of the GATA6 gene (N. 100 
Y. Chia et al. 2015; Lin et al. 2012) which detangles the transcription factor from upstream transcriptional regulation. The chromosome location $18 q 11$ is found to be prone to encounter copy number variations, particularly amplification, and the most important target gene of the copy number increase in this location indeed is GATA6 (Fu et al. 2008; Murphy et al. 2016). Integrated genetic and transcriptional analysis revealed GATA6 amplification in (4/42) 9.5 $\%$ of PDAC samples (Fu et al. 2008) and in $19 \%$ of xenograft specimens (Kwei et al. 2008). GATA6 amplification correlated with an increase in mRNA levels and further translated into increased protein expression (Fu et al. 2008). A recent study by Yue et al observed GATA6 copy number gains in $16 \%$ of PDAC cases, but they also noted that there was a large population of classical PDAC tumors with high GATA6 expression without amplification of the gene (Chan-Seng-Yue et al. 2020). Mechanistically, upon GATA6 amplification, GATA6 positively activates Wnt signaling by negatively regulating the Wnt signalling pathway antagonist DKK1. Furthermore, PDAC patients harboring a GATA6 gain displayed high overall survival (Zhong et al. 2011). However, with regard to GATA6 amplification in PDAC, EZH2 inhibition of those PDAC subtypes might not result in a further increase in GATA6 expression, thus limiting its therapeutic potential. Further studies, for instance in vivo EZH2 inhibition in GATA6 low and GATA6 high PDAC models are required to fully address this hypothesis.

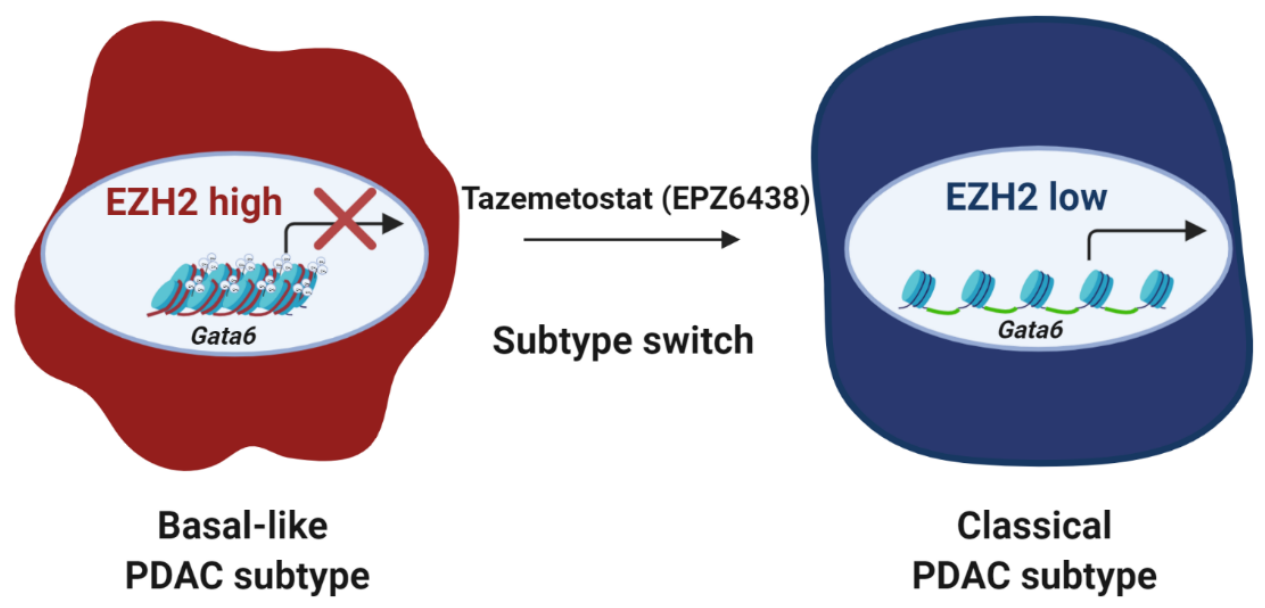

Figure 34: Proposed model depicting subtype switching in PDAC mediated by inhibiting EZH2.

A population of basal-like PDAC exhibits high EZH2 and low GATA6 expression. Upon treating these PDAC with EZH2 inhibitors, e.g. Tazematostat (EPZ6438), EZH2 activity 


\section{DISCUSSION}

is abolished which in turn de-represses GATA6 transcription and mediates switching to a less aggressive classical PDAC subtype (Adapted and modified from Adams et al. 2019).

Taken together, EZH2 inhibition might prove as an advantage in the treatment of a subset of basal-like PDAC subtype patients exhibiting high EZH2 and low GATA6 expression. Therefore, it is important to apply molecular stratification approaches before advising EZH2 inhibition in PDAC therapy.

\subsection{Conclusion}

The key findings of this study are:

1. EZH2 has oncogenic potential in PDAC and mediates tumor progression, dedifferentiation, metastasis and PDAC plasticity.

2. Loss of EZH2 results in the enrichment of gene signatures favoring better prognosis, differentiation and a classical PDAC subtype state.

3. EZH2 transcriptionally represses GATA6, which is a crucial epithelial transcription factor.

4. Genetic or pharmacological inhibition of EZH2 can re-install GATA6 in PDAC cells, which further abrogates tumor-promoting functions of EZH2.

In summary, our findings link transcriptional GATA6 repression to EZH2dependent tumor progression in PDAC and suggest that pharmacological interference with EZH2 activity specifically in a defined subset of PDAC patients can re-install GATA6 expression in favor of a less aggressive and better therapy-responsive tumor phenotype. 


\section{BIBLIOGRAPHY}

Adamo, Patricia et al. 2017. "Profiling Tumour Heterogeneity through Circulating Tumour DNA in Patients with Pancreatic Cancer." Oncotarget 8(50): 87221-33.

Adams, Christina R. et al. 2019. "Transcriptional Control of Subtype Switching Ensures Adaptation and Growth of Pancreatic Cancer." eLife 8: 1-25.

Anders, Simon, Paul Theodor Pyl, and Wolfgang Huber. 2015. "HTSeq-A Python Framework to Work with High-Throughput Sequencing Data." Bioinformatics 31(2): 166-69.

Andricovich, Jaclyn et al. 2018. "Loss of KDM6A Activates Super-Enhancers to Induce Gender-Specific Squamous-like Pancreatic Cancer and Confers Sensitivity to BET Inhibitors." Cancer Cell 33(3): 512-526.

Aranda, Sergi, Gloria Mas, and Luciano Di Croce. 2015. "Regulation of Gene Transcription by Polycomb Proteins." Science Advances 1(11): 1-16.

Artal-Martinez de Narvajas, A. et al. 2013. "Epigenetic Regulation of Autophagy by the Methyltransferase G9a." Molecular and Cellular Biology 33(20): 3983-93.

Audia, James E., and Robert M. Campbell. 2016. "Histone Modifications and Cancer." Cold Spring Harbor Perspectives in Biology 8(4): 1-31.

Aung, Kyaw L et al. 2019. "HHS Public Access Cancer: Early Results from the COMPASS Trial." 24(6): 1344-54.

Bachmann, Ingeborg M. et al. 2006. "EZH2 Expression Is Associated with High Proliferation Rate and Aggressive Tumor Subgroups in Cutaneous Melanoma and Cancers of the Endometrium, Prostate, and Breast." Journal of Clinical Oncology 24(2): 268-73.

Baer, Romain et al. 2014. "Pancreatic Cell Plasticity and Cancer Initiation Induced by Oncogenic Kras Is Completely Dependent on Wild-Type PI 3-Kinase P110a." Genes and Development 28(23): 2621-35.

Bailey, Jennifer M. et al. 2015. "DCLK1 Marks a Morphologically Distinct Subpopulation of Cells with Stem Cell Properties in Pre-Invasive Pancreatic Cancer." 146(1): 245-56.

Bailey, Jennifer M., Kathleen E. DelGiorno, and Howard C. Crawford. 2014. "The Secret Origins and Surprising Fates of Pancreas Tumors." Carcinogenesis 35(7): $1436-40$.

Bailey, Peter et al. 2016. "Genomic Analyses Identify Molecular Subtypes of Pancreatic Cancer." Nature 531(7592): 47-52.

Barton, C. M. et al. 1991. "Abnormalities of the P53 Tumour Suppressor Gene in Human Pancreatic Cancer." British Journal of Cancer 64(6): 1076-81.

Bauer, Ashley J., and Kathleen A. Martin. 2017. "Coordinating Regulation of Gene Expression in Cardiovascular Disease: Interactions between Chromatin Modifiers and Transcription Factors." Frontiers in Cardiovascular Medicine 4:1-19. 


\section{BIBLIOGRAPHY}

Baumgart, Sandra et al. 2014. "Inflammation-Induced NFATc1-STAT3 Transcription Complex Promotes Pancreatic Cancer Initiation by KrasG12D." Cancer Discovery 4(6): 688-701.

Berrozpe, Georgina et al. 1994. "Comparative Analysis of Mutations in the P53 and Kras Genes in Pancreatic Cancer." International Journal of Cancer 58(2): 185-91.

Biankin, Andrew $V$ et al. 2012. "Pancreatic cancer genomes reveal aberrations in axon guidance pathway genes." Nature (7424): 399-405.

Biehs, Brian et al. 2018. "A Cell Identity Switch Allows Residual BCC to Survive Hedgehog Pathway Inhibition." Nature 562(7727): 429-33.

Bishop, Karen S., and Lynnette R. Ferguson. 2015. "The Interaction between Epigenetics, Nutrition and the Development of Cancer." Nutrients 7(2): 922-47.

Borowicz, Stanley et al. 2014. "The Soft Agar Colony Formation Assay." Journal of Visualized Experiments (92): 1-6.

Bracken, Adrian P. et al. 2003. "EZH2 Is Downstream of the PRB-E2F Pathway, Essential for Proliferation and Amplified in Cancer." EMBO Journal 22(20): 5323-35.

Bracken, Adrian P. et al. 2006. "Genome-Wide Mapping of Polycomb Target Genes Unravels Their Roles in Cell Fate Transitions." Genes and Development 20(9): 112336.

Bray, Freddie et al. 2018. "Global Cancer Statistics 2018: GLOBOCAN Estimates of Incidence and Mortality Worldwide for 36 Cancers in 185 Countries." CA: A Cancer Journal for Clinicians 68(6): 394-424.

Bremer, Sebastian C.B. et al. 2019. "Enhancer of Zeste Homolog 2 in Colorectal Cancer Development and Progression." Digestion.

Busnardo, A. C., L. J.A. Didio, R. T. Tidrick, and N. R. Thomford. 1983. "History of the Pancreas." American Journal of Surgery 146(5): 539-50.

Cai, Wesley L. et al. 2020. "Specific Chromatin Landscapes and Transcription Factors Couple Breast Cancer Subtype with Metastatic Relapse to Lung or Brain." BMC Medical Genomics 13(1): 1-18.

Caldas, C et al. 1994. "Frequent Somatic Mutations and Homozygous Deletions of the P16 (MTS1) Gene in Pancreatic Adenocarcinoma." Nature genetics 8(1): 27-32.

Cancer Genome Atlas Research Network et al. 2013. "The Cancer Genome Atlas PanCancer Analysis Project." Nature genetics 45(10): 1113-20.

Chan-Penebre, Elayne et al. 2017. "Selective Killing of SMARCA2- and SMARCA4Deficient Small Cell Carcinoma of the Ovary, Hypercalcemic Type Cells by Inhibition of EZH2: In Vitro and in Vivo Preclinical Models." Molecular Cancer Therapeutics 16(5): 850-60.

Chan-Seng-Yue, Michelle et al. 2020. "Transcription Phenotypes of Pancreatic Cancer Are Driven by Genomic Events during Tumor Evolution." Nature Genetics 52(2): 231240. 
Chen, Jian, Franklin H. Herlong, John R. Stroehlein, and Lopa Mishra. 2016. "Mutations of Chromatin Structure Regulating Genes in Human Malignancies." Current Protein \& Peptide Science 17(5): 411-37.

Chen, Jiangzhi et al. 2014. "Snail Recruits Ring1B to Mediate Transcriptional Repression and Cell Migration in Pancreatic Cancer Cells." Cancer Research 74(16): 4353-63.

Chen, Nai Ming et al. 2017. "Context-Dependent Epigenetic Regulation of Nuclear Factor of Activated T Cells 1 in Pancreatic Plasticity." Gastroenterology 152(6): 15071520.

Chen, Ya Huey, Mien Chie Hung, and Long Yuan Li. 2012. "EZH2: A Pivotal Regulator in Controlling Cell Differentiation." American Journal of Translational Research 4(4): 364-75.

Chen, Ya Tian et al. 2016. "The Novel EZH2 Inhibitor, GSK126, Suppresses Cell Migration and Angiogenesis via down-Regulating VEGF-A." Cancer Chemotherapy and Pharmacology 77(4): 757-65.

Chen, Yang et al. 2018. "Dual Reporter Genetic Mouse Models of Pancreatic Cancer Identify an Epithelial-to-mesenchymal Transition-independent Metastasis Program." EMBO Molecular Medicine 10(10): 1-13.

Cheung, William K C et al. 2013. "Control of Alveolar Differentiation by the Lineage Transcription Factors GATA6 and HOPX Inhibits Lung Adenocarcinoma Metastasis." Cancer cell 23(6): 725-38.

Chia, Crystal Y. et al. 2019. "GATA6 Cooperates with EOMES/SMAD2/3 to Deploy the Gene Regulatory Network Governing Human Definitive Endoderm and Pancreas Formation." Stem Cell Reports 12(1): 57-70.

Chia, Na Yu et al. 2015. "Regulatory Crosstalk between Lineage-Survival Oncogenes KLF5, GATA4 and GATA6 Cooperatively Promotes Gastric Cancer Development." Gut 64(5): 707-19.

Chou, Ruey Hwang, Lian Chiu, Yung Luen Yu, and Woei Cherng Shyu. 2015. "The Potential Roles of EZH2 in Regenerative Medicine." Cell Transplantation 24(3): 31317.

Collisson, Eric A et al. 2011. "Differing Responses to Therapy." Nat med 17(4): 500503.

Cooper, Sarah, and Neil Brockdorff. 2013. "Genome-Wide ShRNA Screening to Identify Factors Mediating Gata6 Repression in Mouse Embryonic Stem Cells." Development (Cambridge) 140(19): 4110-15.

Corradini, N. et al. 2007. "Drosophila Melanogaster as a Model for Studying ProteinEncoding Genes That Are Resident in Constitutive Heterochromatin." Heredity 98(1): 3-12.

Cui, Jing et al. 2015. "EHMT2 Inhibitor BIX-01294 Induces Apoptosis through PMAIP1USP9X-MCL1 Axis in Human Bladder Cancer Cells." Cancer Cell International 15(1): $1-9$. 


\section{BIBLIOGRAPHY}

Daemen, Anneleen et al. 2015. "Metabolite Profiling Stratifies Pancreatic Ductal Adenocarcinomas into Subtypes with Distinct Sensitivities to Metabolic Inhibitors." Proceedings of the National Academy of Sciences of the United States of America 112(32): E4410-17.

Dambacher, S., M. Hahn, and G. Schotta. 2010. "Epigenetic Regulation of Development by Histone Lysine Methylation." Heredity 105(1): 24-37.

Dawkins, Joshua B.N. et al. 2016. "Reduced Expression of Histone Methyltransferases KMT2C and KMT2D Correlates with Improved Outcome in Pancreatic Ductal Adenocarcinoma." Cancer Research 76(16): 4861-71.

Denny, Sarah K. et al. 2016. "Nfib Promotes Metastasis through a Widespread Increase in Chromatin Accessibility." Cell 166(2): 328-42.

Deschamps, Jacqueline et al. 1999. "Initiation, Establishment and Maintenance of Hox Gene Expression Patterns in the Mouse." International Journal of Developmental Biology 43(7): 635-50.

Diaferia, Giuseppe R et al. 2016. " Dissection of Transcriptional and Cis -regulatory Control of Differentiation in Human Pancreatic Cancer ." The EMBO Journal 35(6): 595-617.

Du, Yongxing et al. 2017. "Molecular Subtyping of Pancreatic Cancer: Translating Genomics and Transcriptomics into the Clinic." Journal of Cancer 8(4): 513-22.

Dumartin, L. et al. 2017. "ER Stress Protein AGR2 Precedes and Is Involved in the Regulation of Pancreatic Cancer Initiation." Oncogene 36(22): 3094-3103.

Ellis, Chad A., and Geoff Clark. 2000. "The Importance of Being K-Ras." Cellular Signalling 12(7): 425-34.

Fan, Tao et al. 2012. "EZH2-Dependent Suppression of a Cellular Senescence Phenotype in Melanoma Cells by Inhibition of P21/CDKN1A Expression." Molecular cancer research 9(4): 418-29.

Feber, Andrew et al. 2004. "Amplification and Overexpression of E2F3 in Human Bladder Cancer." Oncogene 23(8): 1627-30.

Fedchenko, Nickolay, and Janin Reifenrath. 2014. "Different Approaches for Interpretation and Reporting of Immunohistochemistry Analysis Results in the Bone Tissue - a Review." Diagnostic pathology 9: 221.

Fedorova, Elena, and Daniele Zink. 2008. "Nuclear Architecture and Gene Regulation." Biochimica et Biophysica Acta - Molecular Cell Research 1783(11): 217484.

Ferlay, J. et al. 2018. "Cancer Incidence and Mortality Patterns in Europe: Estimates for 40 Countries and 25 Major Cancers in 2018." European Journal of Cancer 103: 356-87.

Fisher, J. B., K. Pulakanti, S. Rao, and S. A. Duncan. 2017. "GATA6 Is Essential for Endoderm Formation from Human Pluripotent Stem Cells." Biology Open 6(7): 108495. 
Flavahan, William A., Elizabeth Gaskell, and Bradley E. Bernstein. 2017. "Epigenetic Plasticity and the Hallmarks of Cancer." Science 357(6348).

Fu, Baojin et al. 2008. "Frequent Genomic Copy Number Gain and Overexpression of GATA-6 in Pancreatic Carcinoma." Cancer Biology and Therapy 7(10): 1593-1601.

Fujii, S. et al. 2011. "MEK-ERK Pathway Regulates EZH2 Overexpression in Association with Aggressive Breast Cancer Subtypes." Oncogene 30(39): 4118-28.

Gaudichon, Jérémie et al. 2014. "Deazaneplanocin a Is a Promising Drug to Kill Multiple Myeloma Cells in Their Niche." PLOS ONE 9(9): 1-12.

Glozak, M. A., and E. Seto. 2007. "Histone Deacetylases and Cancer." Oncogene 26(37): 5420-32.

Goll, Mary Grace, and Timothy H. Bestor. 2002. "Histone Modification and Replacement in Chromatin Activation." Genes and Development 16(14): 1739-42.

Gordon-Dseagu, Vanessa L., Susan S. Devesa, Michael Goggins, and Rachael Stolzenberg-Solomon. 2018. "Pancreatic Cancer Incidence Trends: Evidence from the Surveillance, Epidemiology and End Results (SEER) Population-Based Data." International Journal of Epidemiology 47(2): 427-39.

Grassi, Elisa et al. 2018. "Mutational Burden of Resectable Pancreatic Cancer, as Determined by Whole Transcriptome and Whole Exome Sequencing, Predicts a Poor Prognosis." International Journal of Oncology 52(6): 1972-80.

Guerra, Carmen et al. 2007. "Chronic Pancreatitis Is Essential for Induction of Pancreatic Ductal Adenocarcinoma by K-Ras Oncogenes in Adult Mice." Cancer Cell 11(3): 291-302.

Guerra, Carmen, and Mariano Barbacid. 2013. "Genetically Engineered Mouse Models of Pancreatic Adenocarcinoma." Molecular Oncology 7(2): 232-47.

Gulati, Nitya, Wendy Béguelin, and Lisa Giulino-Roth. 2018. "Enhancer of Zeste Homolog 2 (EZH2) Inhibitors." Leukemia \& Lymphoma 59(7): 1574-85.

Gunawan, Merry et al. 2015. "The Methyltransferase Ezh2 Controls Cell Adhesion and Migration through Direct Methylation of the Extranuclear Regulatory Protein Talin." Nature Immunology 16(5): 505-16.

Hahn, Stephan A. et al. 1996. "DPC4, a Candidate Tumor Suppressor Gene at Human Chromosome 18q21.1." Science 271(5247): 350-53.

Han, Ting et al. 2016. "EZH2 Promotes Cell Migration and Invasion but Not Alters Cell Proliferation by Suppressing E-Cadherin, Partly through Association with MALAT-1 in Pancreatic Cancer." Oncotarget 7(10): 11194-207.

Hanahan, Douglas, and Robert A. Weinberg. 2011. "Hallmarks of Cancer: The next Generation." Cell 144(5): 646-74.

Hansel, Donna E., Scott E. Kern, and Ralph H. Hruban. 2003. “ M Olecular P Athogenesis of P Ancreatic C Ancer ." Annual Review of Genomics and Human Genetics 4(1): 237-56. 


\section{BIBLIOGRAPHY}

Hasan, Nesrin, and Nita Ahuja. 2019. 11 Cancers The Emerging Roles of ATPDependent Chromatin Remodeling Complexes in Pancreatic Cancer.

Hauer, Michael H., and Susan M. Gasser. 2017. "Chromatin and Nucleosome Dynamics in DNA Damage and Repair." Genes and Development 31(22): 2204-21.

He, Aibin et al. 2012. "PRC2 Directly Methylates GATA4 and Represses Its Transcriptional Activity." Genes \& development 26(1): 37-42.

Hemming, Sarah et al. 2016. "Identification of Novel EZH2 Targets Regulating Osteogenic Differentiation in Mesenchymal Stem Cells." Stem Cells and Development 25(12): 909-21.

Hermann, Patrick C. et al. 2014. "Nicotine Promotes Initiation and Progression of KRAS-Induced Pancreatic Cancer via Gata6-Dependent Dedifferentiation of Acinar Cells in Mice." Gastroenterology 147(5): 1119-1133.e4.

Hessmann, Elisabeth, Steven A. Johnsen, Jens T. Siveke, and Volker Ellenrieder. 2017. "Epigenetic Treatment of Pancreatic Cancer: Is There a Therapeutic Perspective on the Horizon?" Gut 66(1): 168-79.

Hingorani, Sunil R. et al. 2003. "Preinvasive and Invasive Ductal Pancreatic Cancer and Its Early Detection in the Mouse." Cancer Cell 4(6): 437-50.

Hingorani, Sunil R et al. 2005. "Trp53R172H and KrasG12D Cooperate to Promote Chromosomal Instability and Widely Metastatic Pancreatic Ductal Adenocarcinoma in Mice." Cancer Cell 7(5): 469-83.

Van Holde, K. E. et al. 1980. "DNA-Histone Interactions in Nucleosomes." Biophysical Journal 32(1): 271-82.

Holliday, Robin. 1994. "Epigenetics : An Overview.” Developmental Genetics 453457.

Honma, Daisuke et al. 2017. "Novel Orally Bioavailable EZH1/2 Dual Inhibitors with Greater Antitumor Efficacy than an EZH2 Selective Inhibitor." Cancer Science 108(10): 2069-78.

Hormaeche, Itsaso, and Jonathan D. Licht. 2007. "Chromatin Modulation by Oncogenic Transcription Factors: New Complexity, New Therapeutic Targets." Cancer Cell 11(6): 475-78.

Hruban, R. H. et al. 1993. "K-Ras Oncogene Activation in Adenocarcinoma of the Human Pancreas: A Study of 82 Carcinomas Using a Combination of Mutant-Enriched Polymerase Chain Reaction Analysis and Allele-Specific Oligonucleotide Hybridization." American Journal of Pathology 143(2): 545-54.

Huang, Ling et al. 2015. "Ductal Pancreatic Cancer Modeling and Drug Screening Using Human Pluripotent Stem Cell- and Patient-Derived Tumor Organoids." Nature medicine 21(11): 1364-71.

Hussain, Mustafa et al. 2009. "Tobacco Smoke Induces Polyeomb-Mediated Repression of Dickkopf-1 in Lung Cancer Cells." Cancer Research 69(8): 3570-78.

Hyun, Kwangbeom, Jongcheol Jeon, Kihyun Park, and Jaehoon Kim. 2017. "Writing, Erasing and Reading Histone Lysine Methylations." Experimental and Molecular Medicine 49(4): 324-22. 
lacobuzio-Donahue, Christine A. 2012. "Genetic Evolution of Pancreatic Cancer: Lessons Learnt from the Pancreatic Cancer Genome Sequencing Project." Gut 61(7): 1085-94.

Ideraabdullah, Folami Y., and Steven H. Zeisel. 2018. "Dietary Modulation of the Epigenome." Physiological Reviews 98(2): 667-95.

Iguchi, Eriko et al. 2016. "Pancreatic Cancer, a Mis-Interpreter of the Epigenetic Language." Yale Journal of Biology and Medicine 89(4): 575-90.

Italiano, Antoine et al. 2018. "Tazemetostat, an EZH2 Inhibitor, in Relapsed or Refractory B-Cell Non-Hodgkin Lymphoma and Advanced Solid Tumours: A First-inHuman, Open-Label, Phase 1 Study." The Lancet Oncology 19(5): 649-59.

Jankowska, Anna M et al. 2011. "Mutational Spectrum Analysis of Chronic Myelomonocytic Leukemia Includes Genes Associated with Epigenetic Regulation: UTX, EZH2 \& DNMT3A." Blood 118(14): 3932 - 3941.

Janky, Rekin's et al. 2016. "Prognostic Relevance of Molecular Subtypes and Master Regulators in Pancreatic Ductal Adenocarcinoma." BMC Cancer 16(1): 1-15.

Janzen, William P., Tim J. Wigle, Jian Jin, and Stephen V. Frye. 2010. "Epigenetics: Tools and Technologies." Drug Discovery Today: Technologies 7(1): 59-65.

Jones, Siân et al. 2008. "Core Signaling Pathways in Human Pancreatic Cancer Revealed." Science 321(5897): 1801-6.

Juiz, Natalia Anahi, Juan lovanna, and Nelson Dusetti. 2019. "Pancreatic Cancer Heterogeneity Can Be Explained beyond the Genome." Frontiers in Oncology 9(4): 18.

Kang, Xunlei et al. 2010. "SUMO-Specific Protease 2 Is Essential for Suppression of Polycomb Group Protein-Mediated Gene Silencing during Embryonic Development." Molecular cell 38(2): 191-201.

Kennison, James A. 1995. "THE POL YCOMB AND PROTEINS OF DROSOPHILA: Trans-Regulators of Homeotic Gene Function." Annual Review of Genetics: 289-303.

Ketel, C. S. et al. 2005. "Subunit Contributions to Histone Methyltransferase Activities of Fly and Worm Polycomb Group Complexes." Molecular and Cellular Biology 25(16): 6857-68.

Khuong-Quang, Dong Anh et al. 2012. "K27M Mutation in Histone H3.3 Defines Clinically and Biologically Distinct Subgroups of Pediatric Diffuse Intrinsic Pontine Gliomas." Acta Neuropathologica 124(3): 439-47.

Kim, Daehwan et al. 2013. "TopHat2: Accurate Alignment of Transcriptomes in the Presence of Insertions, Deletions and Gene Fusions." Genome Biology 14(4): R36.

Kim, Kimberly H et al. 2015. "SWI/SNF-Mutant Cancers Depend on Catalytic and NonCatalytic Activity of EZH2." Nature medicine 21(12): 1491-96.

Kim, Shinuk et al. 2014. "Identifying Molecular Subtypes Related to Clinicopathologic Factors in Pancreatic Cancer." BioMedical Engineering Online 13(Suppl 2): S5. 


\section{BIBLIOGRAPHY}

Kleer, Celina G. et al. 2003. "EZH2 Is a Marker of Aggressive Breast Cancer and Promotes Neoplastic Transformation of Breast Epithelial Cells." Proceedings of the National Academy of Sciences of the United States of America 100(20): 11606-11.

Knutson, Sarah K. et al. 2013. "Durable Tumor Regression in Genetically Altered Malignant Rhabdoid Tumors by Inhibition of Methyltransferase EZH2." Proceedings of the National Academy of Sciences of the United States of America 110(19): 7922-27.

Köenig, Alexander et al. 2010. "NFAT-Induced Histone Acetylation Relay Switch Promotes c-Myc-Dependent Growth in Pancreatic Cancer Cells." Gastroenterology 138(3): 1-18.

Kopp, Janel L et al. 2012. "Identification of Sox9-Dependent Acinar-to-Ductal Reprogramming as the Principal Mechanism for Initiation of Pancreatic Ductal Adenocarcinoma." Cancer cell 22(6): 737-50.

Kunnumakkara, Ajaikumar B. et al. 2007. "Curcumin Potentiates Antitumor Activity of Gemcitabine in an Orthotopic Model of Pancreatic Cancer through Suppression of Proliferation, Angiogenesis, and Inhibition of Nuclear Factor-KB-Regulated Gene Products." Cancer Research 67(8): 3853-61.

Kwei, Kevin A. et al. 2008. "Genomic Profiling Identifies GATA6 as a Candidate Oncogene Amplified in Pancreatobiliary Cancer." PLoS Genetics 4(5) 13-20.

Langmead, Ben, and Steven L Salzberg. 2012. "Fast Gapped-Read Alignment with Bowtie 2." Nature Methods 9(4): 357-59.

Lathia, Justin D., and Huiping Liu. 2017. "Overview of Cancer Stem Cells and Stemness for Community Oncologists." Targeted Oncology 12(4): 387-99.

Lee, Ji Min et al. 2012. "EZH2 Generates a Methyl Degron That Is Recognized by the DCAF1/DDB1/CUL4 E3 Ubiquitin Ligase Complex." Molecular Cell 48(4): 572-86.

Li, Gang. 2015. "Patient-Derived Xenograft Models for Oncology Drug Discovery." Journal of Cancer Metastasis and Treatment 5(2): 10-30.

Lin et al. 2012. "Activation of GATA Binding Protein 6 (GATA6) Sustains Oncogenic Lineage-Survival in Esophageal Adenocarcinoma." Proceedings of the National Academy of Sciences of the United States of America 109(11): 4251-56.

Liu, Fan, Lan Wang, Fabiana Perna, and Stephen D. Nimer. 2016. "Beyond Transcription Factors: How Oncogenic Signalling Reshapes the Epigenetic Landscape." Nature Reviews Cancer 16(6): 359-72.

Lomberk, Gwen et al. 2018. "Distinct Epigenetic Landscapes Underlie the Pathobiology of Pancreatic Cancer Subtypes." Nature Communications 9(1): 1-10.

Lomberk, Gwen A., Juan lovanna, and Raul Urrutia. 2016. "The Promise of Epigenomic Therapeutics in Pancreatic Cancer." Epigenomics 8(6): 831-42.

Lorberbaum, David S., and Lori Sussel. 2017. "Gotta Have GATA for Human Pancreas Development." Cell Stem Cell 20(5): 577-79.

Lorenzo, Alessandra Di, and Mark T Bedford. 2011. "Histone Arginine Methylation." FEBS lett 7(1): 66. 
Ma, Anqi et al. 2020. "Discovery of a First-in-Class EZH2 Selective Degrader." Nature chemical biology 16(2): 214-22.

Mahara, Sylvia et al. 2016. "HIFI- $\alpha$ Activation Underlies a Functional Switch in the Paradoxical Role of Ezh2/PRC2 in Breast Cancer." Proceedings of the National Academy of Sciences of the United States of America 113(26): 3735-44.

Mallen-St. Clair, Jon et al. 2012. "EZH2 Couples Pancreatic Regeneration to Neoplastic Progression." Genes and Development 26(5): 439-44.

Mani, Udayakumar, Alagu Sankareswaran S, Arun Goutham R N, and Suma Mohan S. 2017. "SWI/SNF Infobase - An Exclusive Information Portal for SWI/SNF Remodeling Complex Subunits." PLOS ONE 12(9): 1-11.

Margueron, Raphael et al. 2008. "Ezh1 and Ezh2 Maintain Repressive Chromatin through Different Mechanisms." Molecular Cell 32(4): 503-18.

Marmorstein, Ronen, and Ming Ming Zhou. 2014. "Writers and Readers of Histone Acetylation: Structure, Mechanism, and Inhibition." Cold Spring Harbor Perspectives in Biology 6(7): 1-25.

Martens, S. et al. 2019. "Different Shades of Pancreatic Ductal Adenocarcinoma, Different Paths towards Precision Therapeutic Applications." Annals of Oncology 30(9): 1428-36.

Martinelli, Paola et al. 2016. "The Acinar Regulator Gata6 Suppresses KRasG12VDriven Pancreatic Tumorigenesis in Mice." Gut 65(3): 476-86.

Martinelli, Paola et al. 2017. "GATA6 Regulates EMT and Tumour Dissemination, and Is a Marker of Response to Adjuvant Chemotherapy in Pancreatic Cancer." Gut 66(9): 1665-76.

Maurer, Carlo et al. 2019. "Experimental Microdissection Enables Functional Harmonisation of Pancreatic Cancer Subtypes." Gut 68(6): 1034-43.

McCabe, Michael T. et al. 2012. "EZH2 Inhibition as a Therapeutic Strategy for Lymphoma with EZH2-Activating Mutations." Nature 492(7427): 108-12.

McGrath, John, and Patrick Trojer. 2015. "Targeting Histone Lysine Methylation in Cancer." Pharmacology and Therapeutics 150: 1-22.

Mclean, Cory $\mathrm{Y}$ et al. 2010. "GREAT Improves Functional Interpretation of CisRegulatory Regions HHS Public Access Author Manuscript." Nat Biotechnol 28(5): 495-501.

Miele, Evelina et al. 2017. "The Histone Methyltransferase EZH2 as a Druggable Target in SHH Medulloblastoma Cancer Stem Cells." Oncotarget 8(40): 68557-70.

van Mierlo, G., Gert Jan C. Veenstra, Michiel Vermeulen, and Hendrik Marks. 2019. "The Complexity of PRC2 Subcomplexes." Trends in Cell Biology 29(8): 660-71.

Moffitt, Richard A. et al. 2015. "Virtual Microdissection Identifies Distinct Tumor- and Stroma-Specific Subtypes of Pancreatic Ductal Adenocarcinoma." Nature Genetics 47(10): 1168-78. 


\section{BIBLIOGRAPHY}

Morgan, Marc A., and Ali Shilatifard. 2015. "Chromatin Signatures of Cancer." Genes and Development 29(3): 238-49.

Morin, Ryan D et al. 2010. "Somatic Mutations Altering EZH2 (Tyr641) in Follicular and Diffuse Large B-Cell Lymphomas of Germinal-Center Origin." Nature Genetics 42(2): 181-85.

Morris, John et al. 2010. " $\beta$-Catenin Blocks Kras-Dependent Reprogramming of Acini into Pancreatic Cancer Precursor Lesions in Mice." Journal of Clinical Investigation 120(2): 508-20.

Moses, Marsha A., and Di Jia. 2013. "Regulation of Tumor Angiogenesis." Encyclopedia of Systems Biology 18(2): 1834-37.

Mottamal, Madhusoodanan, Shilong Zheng, Tien L. Huang, and Guangdi Wang. 2015. "Histone Deacetylase Inhibitors in Clinical Studies as Templates for New Anticancer Agents." Molecules 20(3): 3898-3941.

Mueller, Sebastian et al. 2018. "Evolutionary Routes and KRAS Dosage Define Pancreatic Cancer Phenotypes." Nature 554(7690): 62-68.

Müller, Jürg et al. 2002. "Histone Methyltransferase Activity of a Drosophila Polycomb Group Repressor Complex." Cell 111(2): 197-208.

Murphy, Stephen J et al. 2016. "Integrated Genomic Analysis of Pancreatic Ductal Adenocarcinomas Reveals Genomic Rearrangement Events as Significant Drivers of Disease." Cancer research 76(3): 749-61.

Musladin, Sanja, Nils Krietenstein, Philipp Korber, and Slobodan Barbaric. 2014. "The RSC Chromatin Remodeling Complex Has a Crucial Role in the Complete Remodeler Set for Yeast PHO5 Promoter Opening." Nucleic Acids Research 42(7): 4270-82.

Nangia-Makker, Pratima, Victor Hogan, and Avraham Raz. 2018. "Galectin-3 and Cancer Stemness." Glycobiology 28(4): 172-81.

Nicolle, Rémy et al. 2017. "Pancreatic Adenocarcinoma Therapeutic Targets Revealed by Tumor-Stroma Cross-Talk Analyses in Patient-Derived Xenografts." Cell reports 21(9): 2458-70.

Nieto, M. Angela, Ruby Yun Y.J. Huang, Rebecca A A. Jackson, and Jean Paul P. Thiery. 2016. "Emt: 2016." Cell 166(1): 21-45.

Ntziachristos, Panagiotis et al. 2012. "Genetic Inactivation of the PRC2 Complex in TCell Acute Lymphoblastic Leukemia." Nature medicine 18(2): 298-301.

O'Kane, Grainne M et al. 2020. "GATA6 Expression Distinguishes Classical and Basal-like Subtypes in Advanced Pancreatic Cancer." Clinical Cancer Research 5(2) :3724.2019.

Olive, Kenneth P., and David A. Tuveson. 2006. "The Use of Targeted Mouse Models for Preclinical Testing of Novel Cancer Therapeutics." Clinical Cancer Research 12(18): 5277-87.

Orth, Michael et al. 2019. "Pancreatic Ductal Adenocarcinoma: Biological Hallmarks, Current Status, and Future Perspectives of Combined Modality Treatment Approaches." Radiation Oncology 14(1): 1-20. 
Ouaïssi, Mehdi et al. 2014. "Further Characterization of HDAC and SIRT Gene Expression Patterns in Pancreatic Cancer and Their Relation to Disease Outcome." PLOS ONE 9(10): 1-14.

Ougolkov, Andrei V, Vladimir N Bilim, and Daniel D Billadeau. 2008. "Regulation of Pancreatic Tumor Proliferation and Chemoresistance by the Histone Methyltransferase EZH2." Clinical Cancer Research 14(21): 6790-96.

Paradise, Brooke D., Whitney Barham, and Martín E. Fernandez-Zapico. 2018. "Targeting Epigenetic Aberrations in Pancreatic Cancer, a New Path to Improve Patient Outcomes?" Cancers 10(5): 1-16.

Pérez-Salvia, Montserrat, and Manel Esteller. 2017. "Bromodomain Inhibitors and Cancer Therapy: From Structures to Applications." Epigenetics 12(5): 323-39.

Polyak, Kornelia, and Robert A. Weinberg. 2009. "Transitions between Epithelial and Mesenchymal States: Acquisition of Malignant and Stem Cell Traits." Nature Reviews Cancer 9(4): 265-73.

Priedigkeit, Nolan et al. 2017. "Intrinsic Subtype Switching and Acquired ERBB2 / HER2 Amplifications and Mutations in Breast Cancer Brain Metastases." JAMA Oncology 3(5): 666-677.

Puleo, Francesco et al. 2018. "Stratification of Pancreatic Ductal Adenocarcinomas Based on Tumor and Microenvironment Features." Gastroenterology 155(6): 19992013.

Puppe, Julian et al. 2009. "BRCA1-Deficient Mammary Tumor Cells Are Dependent on EZH2 Expression and Sensitive to Polycomb Repressive Complex 2-Inhibitor 3Deazaneplanocin A." Breast Cancer Research 11(4): 1-12.

Qazi, Aamer M et al. 2012. "Restoration of E-Cadherin Expression in Pancreatic Ductal Adenocarcinoma Treated with MicroRNA-101." Surgery 152(4): 704-11.

Rahib, Lola et al. 2014. "Projecting Cancer Incidence and Deaths to 2030: The Unexpected Burden of Thyroid, Liver, and Pancreas Cancers in the United States." Cancer Research 74(11): 2913-21.

Raman, Jay D. et al. 2005. "Increased Expression of the Polycomb Group Gene, EZH2, in Transitional Cell Carcinoma of the Bladder." Clinical Cancer Research 11(24): 8570-76.

Ramírez, Fidel et al. 2016. "DeepTools2: A next Generation Web Server for DeepSequencing Data Analysis." Nucleic acids research 44(1): 160-65.

Rao, Zhi Yue et al. 2010. "EZH2 Supports Ovarian Carcinoma Cell Invasion and/or Metastasis via Regulation of TGF- $\beta 1$ and Is a Predictor of Outcome in Ovarian Carcinoma Patients." Carcinogenesis 31(9): 1576-83.

Rawla, Prashanth, Tagore Sunkara, and Vinaya Gaduputi. 2019. "Epidemiology of Pancreatic Cancer: Global Trends, Etiology and Risk Factors." World Journal of Oncology 10(1): 10-27.

Ray, Kevin C. et al. 2011. "Epithelial Tissues Have Varying Degrees of Susceptibility to KrasG12D-Initiated Tumorigenesis in a Mouse Model." PLoS ONE 6(2). 


\section{BIBLIOGRAPHY}

Richart, Laia, and Raphaël Margueron. 2020. "Drugging Histone Methyltransferases in Cancer." Current Opinion in Chemical Biology 56: 51-62.

Richly, H., L. Aloia, and L. Di Croce. 2011. "Roles of the Polycomb Group Proteins in Stem Cells and Cancer." Cell Death and Disease 2(9): 204-7.

Richter, Günther H.S. et al. 2009. "EZH2 Is a Mediator of EWS/FLI1 Driven Tumor Growth and Metastasis Blocking Endothelial and Neuro-Ectodermal Differentiation." Proceedings of the National Academy of Sciences of the United States of America 106(13): 5324-29.

Ringrose, Leonie, and Renato Paro. 2004. "Epigenetic Regulation of Cellular Memory by the Polycomb and Trithorax Group Proteins." Annual Review of Genetics 38(1): 413-43.

Robinson, James T et al. 2011. "Integrative Genome Viewer." Nature Biotechnology 29(1): 24-26.

Rodríguez-García, Antonio, Alberto Sola-Landa, and Carlos Barreiro. 2017. "RNASeq-Based Comparative Transcriptomics: RNA Preparation and Bioinformatics." Methods in molecular biology (Clifton, N.J.) 1645(4): 59-72.

Roe, Jae-seok et al. 2017. "Enhancer Reprogramming Promotes Pancreatic Cancer Metastasis." Cell 170(5): 875-888.

Ross-Innes, Caryn S. et al. 2012. "Differential Oestrogen Receptor Binding Is Associated with Clinical Outcome in Breast Cancer." Nature 481(7381): 389-93.

Rozenblum, Ester et al. 1997. "Tumor-Suppressive Pathways in Pancreatic Carcinoma." Cancer Research 57(9): 1731-34.

De Ruijter, Annemieke J.M. et al. 2003. "Histone Deacetylases (HDACs): Characterization of the Classical HDAC Family." Biochemical Journal 370(3): 737-49.

Russo, Alicia A. et al. 1998. "Structural Basis for Inhibition of the Cyclin-Dependent Kinase Cdk6 by the Tumour Suppressor P16(INK4a)." Nature 395(6699): 237-43.

Sahu, R. P., S. Batra, and S. K. Srivastava. 2009. "Activation of ATM/Chk1 by Curcumin Causes Cell Cycle Arrest and Apoptosis in Human Pancreatic Cancer Cells." British Journal of Cancer 100(9): 1425-33.

San, Bilge et al. 2016. "Normal Formation of a Vertebrate Body Plan and Loss of Tissue Maintenance in the Absence of Ezh2." Scientific Reports 6: 1-16.

Sauvageau, Martin, and Guy Sauvageau. 2010. "Polycomb Group Proteins: MultiFaceted Regulators of Somatic Stem Cells and Cancer." Cell Stem Cell 7(3): 299-313.

Schuettengruber, Bernd et al. 2007. "Genome Regulation by Polycomb and Trithorax Proteins." Cell 128(4): 735-45.

Schuettengruber, Bernd, and Giacomo Cavalli. 2009. "Recruitment of Polycomb Group Complexes and Their Role in the Dynamic Regulation of Cell Fate Choice." Development 136(21): 3531-42. 
Seino, Takashi et al. 2018. "Human Pancreatic Tumor Organoids Reveal Loss of Stem Cell Niche Factor Dependence during Disease Progression." Cell Stem Cell 22(3): 454-467.

Shi, B. et al. 2007. "Integration of Estrogen and Wnt Signaling Circuits by the Polycomb Group Protein EZH2 in Breast Cancer Cells." Molecular and Cellular Biology 27(14): 5105-19.

Shin, Hyunjin, Tao Liu, Arjun K. Manrai, and Shirley X. Liu. 2009. "CEAS: CisRegulatory Element Annotation System." Bioinformatics 25(19): 2605-6.

Shlush, Liran I. et al. 2017. "Tracing the Origins of Relapse in Acute Myeloid Leukaemia to Stem Cells." Nature 547(7661): 104-8.

Singh, Rahul, Katie Reindl, and Rick Jansen. 2019. "Epigenomics of Pancreatic Cancer: A Critical Role for Epigenome-Wide Studies." Epigenomes 3(1): 5.

Smit, V. T.H.B.M. et al. 1988. "KRAS Codon 12 Mutations Occur Very Frequently in Pancreatic Adenocarcinomas." Nucleic Acids Research 16(22): 10952.

Smith, Keriayn N, Amar M Singh, and Stephen Dalton. 2010. "Myc Represses Primitive Endoderm Differentiation in Pluripotent Stem Cells." Cell stem cell 7(3): 34354.

Smits, Michiel et al. 2010. "MiR-101 Is down-Regulated in Glioblastoma Resulting in EZH2- Induced Proliferation, Migration, and Angiogenesis AbstrAct: Background: Glioblastoma ( GBM ) Is a Malignant Brain Tumor with Dismal." Oncotarget 1(8): 71020.

Solcia, E., M. Bonato, and G. N. Ranzani. 1994. "K-Ras and P53 Gene Mutations in Pancreatic Cancer: Ductal and Nonductal Tumors Progress through Different Genetic Lesions." Cancer Research 54(6): 1556-60.

Sparmann, Anke, and Maarten Van Lohuizen. 2006. "Polycomb Silencers Control Cell Fate, Development and Cancer." Nature Reviews Cancer 6(11): 846-56.

Stenzinger, Albrecht et al. 2013. "High SIRT1 Expression Is a Negative Prognosticator in Pancreatic Ductal Adenocarcinoma." BMC Cancer 13(1): 1-10.

Sterner, D. E., and S. L. Berger. 2000. "Acetylation of Histones and TranscriptionRelated Factors." Microbiology and Molecular Biology Reviews 64(2): 435-59.

Subramanian, Aravind et al. 2005. "Gene Set Enrichment Analysis: A KnowledgeBased Approach for Interpreting Genome-Wide Expression Profiles." Proceedings of the National Academy of Sciences of the United States of America 102(43): 15545-50.

Sudo, T. et al. 2005. "Clinicopathological Significance of EZH2 MRNA Expression in Patients with Hepatocellular Carcinoma." British Journal of Cancer 92(9): 1754-58.

Sun, Bin, Eunhyuk Chang, Anna Gerhartl, and Francis G. Szele. 2018. "Polycomb Protein Eed Is Required for Neurogenesis and Cortical Injury Activation in the Subventricular Zone." Cerebral Cortex 28(4): 1369-82.

Surface, Lauren E., Seraphim R. Thornton, and Laurie A. Boyer. 2010. "Polycomb Group Proteins Set the Stage for Early Lineage Commitment." Cell Stem Cell 7(3): 288-98. 


\section{BIBLIOGRAPHY}

Suzuki, Shinnosuke et al. 2015. "Histone Methyltransferase Smyd3 Regulates Early Embryonic Lineage Commitment in Mice." Reproduction 150(1): 21-30.

Tammen, Stephanie A., Simonetta Friso, and Sang Woon Choi. 2013. "Epigenetics: The Link between Nature and Nurture." Molecular Aspects of Medicine 34(4): 753-64.

Tan, Jing et al. 2007. "Pharmacologic Disruption of Polycomb-Repressive Complex 2Mediated Gene Repression Selectively Induces Apoptosis in Cancer Cells." Genes and Development 21(9): 1050-63.

Tavakkoli, Anna et al. 2020. "Racial Disparities and Trends in Pancreatic Cancer Incidence and Mortality in the United States." Clinical Gastroenterology and Hepatology 18(1): 171-178.

The Cancer Genome Atlas Research Network, and Benjamin J Raphael. 2017. "Integrated Genomic Characterization of Pancreatic Ductal Adenocarcinoma The Cancer Genome Atlas Research Network *." Cancer Cell 32(2)(2): 185-203.

Thilo Welsch, Jorg Kleeff, and Helmut Friess. 2007. "Molecular Pathogenesis of Pancreatic Cancer: Advances and Challenges." Current Molecular Medicine 7(5): 50421.

Thinnes, Cyrille C. et al. 2014. "Targeting Histone Lysine Demethylases - Progress, Challenges, and the Future." Biochimica et Biophysica Acta - Gene Regulatory Mechanisms 1839(12): 1416-32.

Thorvaldsdóttir, Helga, James T. Robinson, and Jill P. Mesirov. 2013. "Integrative Genomics Viewer (IGV): High-Performance Genomics Data Visualization and Exploration." Briefings in Bioinformatics 14(2): 178-92.

Tiyaboonchai, Amita et al. 2017. "GATA6 Plays an Important Role in the Induction of Human Definitive Endoderm, Development of the Pancreas, and Functionality of Pancreatic $\beta$ Cells." Stem Cell Reports 8(3): 589-604.

Toll, Adam D. et al. 2010. "Implications of Enhancer of Zeste Homologue 2 Expression in Pancreatic Ductal Adenocarcinoma." Human Pathology 41(9): 1205-9.

Trapnell, Cole et al. 2013. "Differential Analysis of Gene Regulation at Transcript Resolution with RNA-Seq." Nature Biotechnology 31(1): 46-53.

Tyagi, Monica, Nasir Imam, Kirtika Verma, and Ashok K. Patel. 2016. "Chromatin Remodelers: We Are the Drivers!!" Nucleus 7(4): 388-404.

Tzatsos, Alexandros et al. 2013. "KDM2B Promotes Pancreatic Cancer via PolycombDependent and -Independent Transcriptional Programs." Journal of Clinical Investigation 123(2): 727-39.

Upadhyay, Anup K., and Xiaodong Cheng. 2011. "Dynamics of Histone Lysine Methylation: Structures of Methyl Writers and Erasers." Progress in Drug Research 67: 107-24.

Valk-Lingbeek, Merel E., Sophia W.M. Bruggeman, and Maarten Van Lohuizen. 2004. "Stem Cells and Cancer: The Polycomb Connection." Cell 118(4): 409-18.

Vanharanta, Sakari et al. 2013. "Epigenetic Expansion of VHL-HIF Signal Output Drives Multiorgan Metastasis in Renal Cancer." Nature medicine 19(1): 50-56. 
Varambally, Sooryanarayana et al. 2002. "The Polycomb Group Protein EZH2 Is Involved in Progression of Prostate Cancer." Nature 419(6907): 624-29.

Vaswani, Rishi G. et al. 2016. "Identification of (R)-N-((4-Methoxy-6-Methyl-2-Oxo-1,2Dihydropyridin-3-YI)Methyl)-2-Methyl-1-(1-(1-(2,2,2-Trifluoroethyl)Piperidin-4-YI)Ethyl)$1 \mathrm{H}$-Indole-3-Carboxamide (CPI-1205), a Potent and Selective Inhibitor of Histone Methyltransferase EZH2, Suitabl." Journal of Medicinal Chemistry 59(21): 9928-41.

Virani, Shama et al. 2012. "Cancer Epigenetics: A Brief Review." ILAR journal / National Research Council, Institute of Laboratory Animal Resources 53(3-4): 359-69.

Völkel, Pamela, Barbara Dupret, Xuefen Le Bourhis, and Pierre Olivier Angrand. 2015. "Diverse Involvement of EZH2 in Cancer Epigenetics." American Journal of Translational Research 7(2): 175-93.

Wang, Hui, Anna Paczulla, and Claudia Lengerke. 2015. "Evaluation of Stem Cell Properties in Human Ovarian Carcinoma Cells Using Multi and Single Cell-Based Spheres Assays." Journal of Visualized Experiments (95): 1-11.

Wang, Pingting et al. 2018. "KDM6A Promotes Chondrogenic Differentiation of Periodontal Ligament Stem Cells by Demethylation of SOX9." Cell Proliferation 51(3): $1-13$.

Wilson, Boris G et al. 2010. "Epigenetic Antagonism between Polycomb and SWI/SNF Complexes during Oncogenic Transformation." Cancer Cell 18(4): 316-28.

Xu, Cong et al. 2019. "Patient-Derived Xenograft Mouse Models: A High Fidelity Tool for Individualized Medicine (Review)." Oncology Letters 17(1): 3-10.

$\mathrm{Xu}$, K. et al. 2012. "EZH2 Oncogenic Activity in Castration-Resistant Prostate Cancer Cells Is Polycomb-Independent." Science 338(6113): 1465-69.

Xu, Yali, and Christopher R Vakoc. 2017. "Targeting Cancer Cells with BET Bromodomain Inhibitors." Cold Spring Harbor Perspectives in Biology: 1-17.

Yachida, Shinichi, and Christine A. Lacobuzio-Donahue. 2009. "The Pathology and Genetics of Metastatic Pancreatic Cancer." Archives of Pathology and Laboratory Medicine 133(3): 413-22.

Yadav, Dhiraj, and Albert B Lowenfels. 2013. "The Epidemiology of Pancreatitis and Pancreatic Cancer." Gastroenterology 144(6): 1252-61.

Ye, Jie et al. 2019. "PRDM3 Attenuates Pancreatitis and Pancreatic Tumorigenesis by Regulating Inflammatory Response." Cell Death Dis 11(3): 187.

Yoon, Kyong Ah et al. 2019. "Comprehensive Cancer Panel Sequencing Defines Genetic Diversity and Changes in the Mutational Characteristics of Pancreatic Cancer Patients Receiving Neoadjuvant Treatment." Gut and Liver 13(6): 683-89.

Young, Claire C. et al. 2019. "The Loss of ATRX Increases Susceptibility to Pancreatic Injury and Oncogenic KRAS in Female But Not Male Mice." Cmgh 7(1): 93-113.

Yuan, Yuan et al. 2012. "A Small-Molecule Probe of the Histone Methyltransferase G9a Induces Cellular Senescence in Pancreatic Adenocarcinoma." ACS Chemical Biology 7(7): 1152-57. 


\section{BIBLIOGRAPHY}

Yun, Miyong, Jun Wu, Jerry L. Workman, and Bing Li. 2011. "Readers of Histone Modifications." Cell Research 21(4): 564-78.

Zeeshan, Rabia, and Zeeshan Mutahir. 2017. "Cancer Metastasis - Tricks of the Trade." Bosnian Journal of Basic Medical Sciences 17(3): 172-82.

Zhang, Yong et al. 2008. "Model-Based Analysis of ChIP-Seq (MACS)." Genome Biology 9(9): 137.

Zheng, Xiaofeng et al. 2015. "EMT Program Is Dispensable for Metastasis but Induces Chemoresistance in Pancreatic Cancer." Nature 527(7579): 525-30.

Zhong, Yi et al. 2011. "GATA6 Activates Wnt Signaling in Pancreatic Cancer by Negatively Regulating the Wnt Antagonist Dickkopf-1." PLOS ONE 6(7): 22129.

Zhou, Qiao, and Douglas A Melton. 2018. "Pancreas Regeneration." Nature 557(7705): 351-58.

Zingg, Daniel et al. 2015. "The Epigenetic Modifier EZH2 Controls Melanoma Growth and Metastasis through Silencing of Distinct Tumour Suppressors." Nature Communications 6(5): 6061. 
ABBREVIATIONS

\begin{tabular}{|l|l|}
\hline$a$ & alpha \\
\hline$\mu$ & micro \\
\hline ADM & acinar-to-ductal metaplasia \\
\hline ADEX & Aberrantly differentiated endocrine exocrine \\
\hline AEBP2 & Adipocyte enhancer binding protein 2 \\
\hline ANOVA & Analysis of variance \\
\hline APS & Ammonium persulfate \\
\hline ATAC-seq & $\begin{array}{l}\text { Assay for transposase-accessible chromatin with high- } \\
\text { throughput sequencing }\end{array}$ \\
\hline ATP & Adenosine triphosphate \\
\hline BRCA1 & Breast cancer 1 \\
\hline BrdU & Bromodeoxyuridine \\
\hline BSA & Bovine serum albumin \\
\hline CDH1 & E-Cadherin \\
\hline CDKN2A & Cyclin dependent kinase inhibitor 2A \\
\hline cDNA & Complementary DNA \\
\hline CDX & Cells derived from xenograft \\
\hline CEAS & Cir-regulatory element annotation system \\
\hline CHD & Chromodomain helicase DNA-binding \\
\hline ChIP & Chromatin immunoprecipitation \\
\hline ChIP-seq & ChIP followed by deep sequencing \\
\hline CO2 & Carbondioxide \\
\hline CRC & Colorectal cancer \\
\hline CRISPR & Clustered regularly interspaced short palindromic repeats \\
\hline CXCR4 & C-X-C motif chemokine receptor 4 \\
\hline DAPI & 4 ',6-diamidino-2-phenylindole \\
\hline DLBCL & Diffuse large b cell lymphoma \\
\hline DMEM & Dulbecco's modified eagle medium \\
\hline DMSO & Dimethyl sulfoxide \\
\hline DNA & \\
\hline DZnep & Deoxyribo nucleic acid \\
\hline
\end{tabular}




\section{ABBREVIATIONS}

\begin{tabular}{|l|l|}
\hline DTT & dithiothreitol \\
\hline ECM & Extracellular matrix \\
\hline EDTA & Ethylenediaminetetraacetic acid \\
\hline EED & Embryonic ectoderm development \\
\hline EGF & Epidermal growth factor \\
\hline EGTA & $\begin{array}{l}\text { Ethylene glycol-bis( } \beta \text {-aminoethyl ether)-N,N,N',N'-tetraacetic } \\
\text { acid }\end{array}$ \\
\hline EMT & Epithelial to mesenchymal transition \\
\hline ENKC & EZH2 ${ }^{\text {fl/ }} ;$ caNFATc1;Kras ${ }^{\text {G12D }}$ \\
\hline ERBB2 & Erythroblastic oncogene B \\
\hline ERK & Extracellular-signal-regulated kinase \\
\hline EZH2 & Enhancer of zeste homologue 2 \\
\hline FCS & Fetal calf serum \\
\hline FDA & Food and drug administration \\
\hline FDR & False discovery rate \\
\hline FGF & Fibroblast growth factor \\
\hline FP & Forward primer \\
\hline FPKM & Fragment per kilobase of transcript per million \\
\hline FL & Follicular lymphoma \\
\hline fl & Floxed \\
\hline FU & Fluorouracil \\
\hline GCDX & Goettingen cell derived xenograft \\
\hline GEMM & Genetically engineered mouse models \\
\hline GO & Gene ontology \\
\hline GOF & Gain-of-function \\
\hline GREAT & Genomin regions enrichment of annotations tool \\
\hline GSEA & Gene set enrichment analysis \\
\hline GTP & Guanidine triphosphate \\
\hline H3 & Histone 3 \\
\hline H3K4me3 3 lysine 4 trimethylation \\
\hline H3K27ac & H3K27me3 trimethylation \\
\hline & Histone 7 acetylation \\
\hline
\end{tabular}




\begin{tabular}{|l|l|}
\hline HA & Hemagglutinin \\
\hline HAT & Histone acetyl transferase \\
\hline HDAC & Histone deacetylase \\
\hline HE & Hematoxylin \& eosin \\
\hline HER2 & Human epidermal growth factor receptor 2 \\
\hline HNF & Hepatocyte nuclear factor \\
\hline HOX & Homeobox \\
\hline HRP & Horse raddish peroxidase \\
\hline IgG & Immunoglobulin G \\
\hline IGV & Integrative genome viewer \\
\hline IHC & Immunohistochemistry \\
\hline INI & Integrase interactor 1 \\
\hline IP & Immunoprecipitation \\
\hline IRS & Immune reactive score \\
\hline ISWI & Imitation SWI \\
\hline JARID & Jumonji and AT rich interaction domain 2 \\
\hline KAT & Lysine acetyl transferase \\
\hline Kb & Kilobase \\
\hline KCI & Potassium chloride \\
\hline KDa & Kilo Dalton \\
\hline KH2PO4 & Potassium dihydrogen phosphate \\
\hline KMT & Lysine methyltransferase \\
\hline KPC & Kras G12D;TP53172H/+ \\
\hline KRAS & Kirsten rat sarcoma \\
\hline KSF & Keratinocyte serum-free \\
\hline LiCI & Lithium chloride \\
\hline LOF & Loss-of-function \\
\hline log2FC & Log2 fold change \\
\hline MACS & Model-based analysis of ChIP-seq \\
\hline MAPK & Mitogen-activated protein kinase \\
\hline MEK & Minase \\
\hline miRNA & \\
\hline
\end{tabular}




\section{ABBREVIATIONS}

\begin{tabular}{|c|c|}
\hline MLL & Mixed lineage leukemia \\
\hline MPN & Myeloproliferative neoplasms \\
\hline mRNA & Messenger RNA \\
\hline N2 & Nitrogen supplement \\
\hline $\mathrm{Na} 2 \mathrm{HPO} 4$ & Disodium hydrogen phosphate \\
\hline $\mathrm{NaCl}$ & Sodium chloride \\
\hline $\mathrm{NaF}$ & Sodium fluride \\
\hline $\mathrm{NaO}$ & Sodium oxide \\
\hline NEAA & Non essential amino acids \\
\hline NES & Normalized enrichment score \\
\hline NFAT & Nuclear factor of activated T cells \\
\hline NGS & Next generation sequencing \\
\hline NIG & NGS integrative genomics \\
\hline NKC & caNFATc1;Kras $^{\mathrm{G} 12 \mathrm{D}}$ \\
\hline NP40 & Nonidet P-40 \\
\hline OD & Optical density \\
\hline PAGE & Polyacrylamide gel electrophoresis \\
\hline PanIN & Pancreatic intraepithelial neoplasia \\
\hline PANTHER & Protein analysis through evolutionary relationships \\
\hline PBS & Phosphate-buffered saline \\
\hline PBST & PBS with tween20 or triton-X \\
\hline PCA & Principal component analysis \\
\hline PcG & Polycomb group \\
\hline PCR & Polymerase chain reaction \\
\hline PDAC & Pancreatic ductal adenocarcinoma \\
\hline PDTX & Patient derived tumor xenograft \\
\hline PDX & Patient derive xenograft \\
\hline PFA & Paraformaldehyde \\
\hline PMSF & Phenylmethylsulfonyl fluoride \\
\hline PRC & Polycomb repressive complex \\
\hline PRMT & Arginine methyltransferase \\
\hline QM & Quasi-mesenchymal \\
\hline
\end{tabular}




\begin{tabular}{|l|l|}
\hline qPCR & Quantitative PCR \\
\hline Rb & Retinoblastoma \\
\hline RBBP4/7 & Retinoblastoma binding protein 4/7 \\
\hline RNA & Ribonucleic acid \\
\hline RNA ISH & RNA in situ hybridization \\
\hline RNA-seq & RNA sequencing \\
\hline RORa & RAR-relate orphan receptor \\
\hline RP & Reverse primer \\
\hline RPM & Rotations per minute \\
\hline RT & Room temperature \\
\hline SAH & S-adenosyl homocysteine \\
\hline SD & Standard deviation \\
\hline SDS & Sodium dodecyl sulfate \\
\hline SET & Su(var)3-9,EZH2 and Trithorax \\
\hline sgRNA & Single guide RNA \\
\hline siRNA & Small interfering RNA \\
\hline shRNA & Small hair pin RNA \\
\hline SMARCA & $\begin{array}{l}\text { SWI/SNF-related } \\
\text { regulator of chromatin subfamily A }\end{array}$ \\
\hline SUZ12 & Suppressor of zeste 12 \\
\hline SWI/SNF & Switch/sucrose non-fermentable \\
\hline T-ALL & T-cell acute lymphoblastic leukemia \\
\hline TBS & Tris-buffered saline \\
\hline TBST & TBS with tween20 or triton-X \\
\hline TCGA & The cancer genome atlas \\
\hline TE & Tris EDTA \\
\hline TEMED & tetramethylethylenediamine \\
\hline TMA & Tissue microarray \\
\hline TP53 & Tumor protein 53 \\
\hline trxG & \\
\hline TSS & Trithorax group \\
\hline UTR & acription start site \\
\hline
\end{tabular}


ABBREVIATIONS

\begin{tabular}{|l|l|}
\hline WB & Weinmann buffer \\
\hline wt & Wild type \\
\hline
\end{tabular}




\section{APPENDIX}

\section{APPENDIX}

We established CRISPR/Cas9 mediated knockout of Ezh2 in NKC cells. The CRISPR/Cas9 control and CRISPR/Cas9 Ezh2 NKC cells were given for sequencing to confirm the knockout of Ezh2 at the genome level. Further, we isolated the lysate from these cells and validated the absence of EZH2 in the western blot. The cells were seeded in triplicates and harvested for RNA. Further, they were subjected to high throughput RNA sequencing. Before proceeding with the analysis, we carried out the quality check and Principal component analysis (PCA) through which we confirmed the similarity between the triplicates of each condition and the differences between the two conditions (Figure 35). 


\section{APPENDIX}

(A)
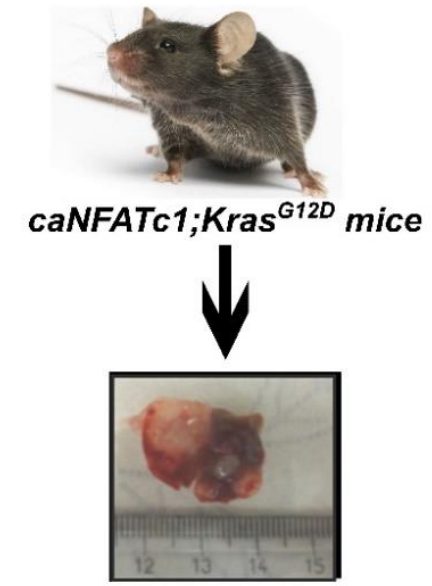

PDAC tumor

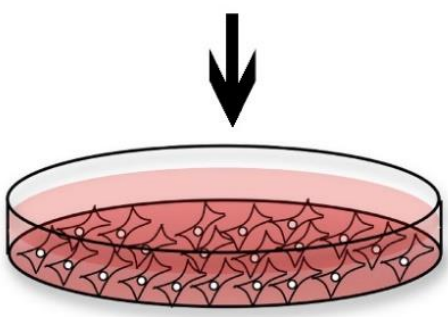

Primary PDAC cells

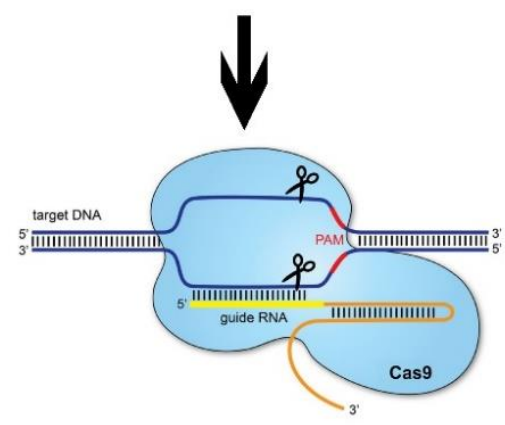

(B)

CTGAAAATAAGTCACTGGATTATCTATGTTTTTCACTTTAGATTTTFTG I1 11111111111111111111111111111111111111111111111 TGGATGCAACCCGAAAGGGCAACAAAATTCGTTTTGCTAATCATTCAGTA

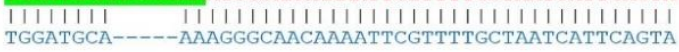

(C)

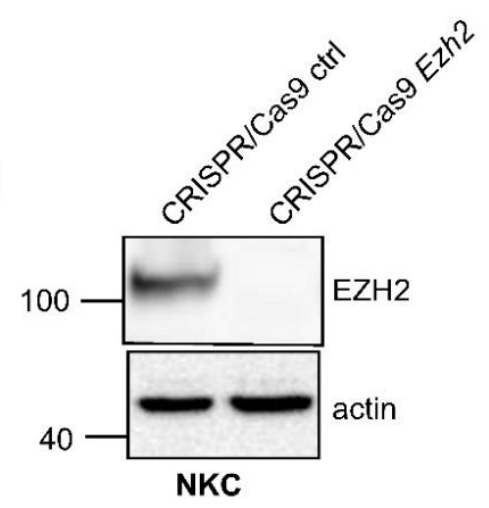

(D)

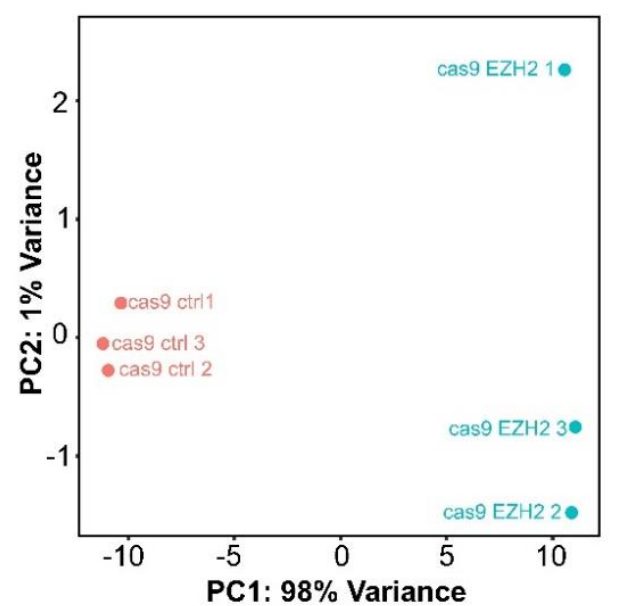

Figure 35: Generation of CRISPR/Cas9 mediated stable knockout of EZH2 in NKC cells.

(A) Schematic displaying the isolation of primary PDAC cells (NKC cells) from caNfatc1;Kras ${ }^{G 12 D}$ mice and further generation of CRISPR/Cas9 mediated stable knockdown of Ezh2 in these cells. (B) Nucleotide alignment confirming CRISPR/Cas9mediated knockout of Ezh2 in NKC cells. The position where the guide RNA interferes is highlighted in green. Red: sequence of CRISPR/Cas9 control cells; blue: sequence of CRISPR/Cas9 Ezh2 cells. (C) Western blot analysis in indicated cells confirming absence of Ezh2 expression in NKC cells upon CRISPR/Cas9 mediated Ezh2 knockout. Actin is used as the house keeping gene. (D) PCA plot from RNA-seq analysis in CRISPR/Cas9 control and CRISPR/Cas9 Ezh2 NKC cells showing the triplicates of both the clones cluster separately. 


\section{CURRICULUM VITAE}

SHILPA PATIL

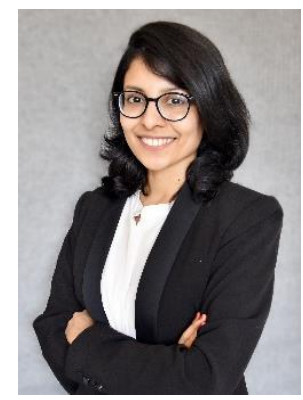

\section{PERSONAL DETAILS:}

Address: Goettingen, Lower Saxony, Germany 37075

Date of birth: 28.05.1991

Place of birth: Manipal, India

E-mail address: shilpapatil528@gmail.com

Contact number: +4917657979548

\section{EDUCATIONAL QUALIFICATIONS:}

Doctor of Philosophy (Ph.D.) University Medical Center, Goettingen, Germany Pancreatic Cancer May 2016- July 2020 (expected)

Thesis Title: EZH2-GATA6 axis in Pancreatic ductal adenocarcinoma.

I am studying the mechanistic and functional role of EZH2 (Enhancer of Zeste Homologue 2) in tumor progression, plasticity and molecular subtype identity of pancreatic cancer. My work suggests GATA6 as the crucial target of EZH2 and elaborate on the EZH2-GATA6 axis in regulating classical PDAC subtype.

Master of Science (Research) (M.Sc.)

Regenerative Medicine (72\%)

Manipal University, Bangalore, India

2012-2014

Thesis Title: Analysis of copy number variation in chronic myeloid leukemia (CML).

(In National center Biological Sciences (NCBS) and St John's Medical college, Bangalore, India)

In this study, we discovered a novel strategy to predict if the CML patients are responders or nonresponders to the imatinib therapy, based on copy number variation of a few vital genes in their bone marrow samples.

Bachelor of Science (B.Sc.)

Manipal University, Manipal, India Biotechnology (80\%) 2009-2012

Thesis Title: Alterations in the extracellular matrix (ECM) proteins under hypoxic conditions in HepG2 cells.

In this project, we studied the differences in the expression pattern of ECM proteins in normoxic and hypoxic conditions in HepG2 cells using Mass Spectrometry based proteomics tool. We identified novel proteins differentially expressed upon hypoxia. 


\section{WORK EXPERIENCE:}

Research Assistant

JNCASR, India

Sept 2014 - Feb 2016

I worked on a project aimed at investigating the factors mediating p300 autoacetylation. The main idea was to understand the effect of wildtype and various mutants of p53 to mediate p300 autoacetylation. I was also involved in a project in elucidating the mechanisms behind NPM1 mediating p300 autoacetylation.

\section{MENTORSHIP SKILLS:}

- Guided two Medical students on lab rotations, one month each with basic lab techniques

- Conducted a method course on 'Mouse models of pancreatic cancer - Tumor screening by ultrasound' (Part of method courses offered by Molecular medicine program) at UMG, Goettingen in 2017.

- Mentored a one-day workshop on 'Orthotopic transplantation of pancreatic tumor cells into pancreas of mice' (Workshop offered as a part of Deutsche Pancreas Club (DPC) conference hosted in Goettingen in 2019).

- Provided regular teaching/guidance on orthotopic transplantation and ultrasoundbased tumor screening techniques in mice.

\section{PUBLICATIONS:}

EZH2 regulates pancreatic cancer subtype identity and tumor progression by transcriptional repression of GATA6 Patil $\mathbf{S}$ et al... Hessmann E. (manuscript in revision)

Preclinical Evaluation of 1,2-Diamino-4,5-Dibromobenzene in Genetically Engineered Mouse Models of Pancreatic Cancer. Goetze, Robert G., Buchholz, S. M., Ou, N., Zhang, Q., Patil, S., Schirmer, M., ... Neesse, A. (2019). Cells, 8(6), 563.

Cytosolic 5'-nucleotidase $1 \mathrm{~A}$ is overexpressed in pancreatic cancer and mediates gemcitabine resistance by reducing intracellular gemcitabine metabolites. Patzak, $M$. S., Kari, V., Patil, S., Hamdan, F. H., Goetze, R. G., Brunner, M., ... Neesse, A. (2019). EBioMedicine, 40, 394-405.

Mutant and Wild-Type Tumor Suppressor p53 Induces p300 Autoacetylation. Kaypee, S., Sahadevan, S. A., Patil, S., Ghosh, P., Roy, N. S., Roy, S., \& Kundu, T. K. (2018). IScience, 4, 260-272.

Oligomers of human histone chaperone NPM1 alter p300/KAT3B folding to induce autoacetylation. Kaypee, S., Sahadevan, S. A., Sudarshan, D., Halder Sinha, S., Patil, S., Senapati, P., ... Kundu, T. K. (2018). Biochimica et Biophysica Acta. General Subjects, 1862(8), 1729-1741. 


\section{CURRICULUM VITAE}

Utilizing high resolution ultrasound to monitor tumor onset and growth in genetically engineered pancreatic cancer models. Goetze, Robert Guenther, Buchholz, S. M.,

Patil, S., Petzold, G., Ellenrieder, V., Hessmann, E., \& Neesse, A. (2018). Journal of Visualized Experiments, 2018(134).

\section{CONFERENCES:}

- Participated and presented poster in the European Pancreas Club (EPC) conferences in 2019 (held at Bergen, Norway) \& 2017 (held at Budapest, Hungary).

- Participated and presented poster in the Deutsche Pancreas Club (DPC) conferences in 2019 (Goettingen, Germany), 2018 (held at Ulm, Germany) \& 2017 (Greifswald, Germany). Also volunteered in organizing the DPC held in 2019 at Goettingen, Germany.

- Participated, presented poster and volunteered in organizing the Pancreas spring meeting held in Goettingen, Germany in 2017.

- Participated, presented poster and volunteered in organizing the EKFS cancer symposium held in Goettingen, Germany in 2017.

- Participated and volunteered in organizing the international conference 'Gene networks in chromatin/chromosome function, $5^{\text {th }}$ meeting of the Asian forum of chromosome and chromatin biology' held at JNCASR, Bangalore in 2015.

\section{ACADEMIC MERITS:}

- Awarded the Best Abstract Prize for the poster titled 'Characterization of EZH2 in PDAC plasticity' at Deutsche Pancreas Club (DPC) conference held at Ulm, Germany in Jan 2018.

- Awarded the travel award for the poster titled 'Role of EZH2 in PDAC' at European Pancreas Club (EPC) conference held at Budapest, Hungary in June 2017.

- Awarded Dr. TMA Pai Gold Medal for the best outgoing graduate student of Manipal Life Sciences Centre, Manipal University in 2012.

- Awarded the Certificate of Merit for Outstanding Academic Performance and for being among the top $0.1 \%$ of successful candidates of All India Secondary Examinations in English Language and literature in 2007.

\section{TEST SCORES:}

- Obtained IELTS overall score 7.5 (2019). 